University of Louisville

ThinkIR: The University of Louisville's Institutional Repository

Electronic Theses and Dissertations

$5-2004$

\title{
The transcriptional repressor protein growth factor independence-1B in T lymphopoiesis.
}

Loretta L. Doan

University of Louisville

Follow this and additional works at: https://ir.library.louisville.edu/etd

\section{Recommended Citation}

Doan, Loretta L., "The transcriptional repressor protein growth factor independence-1B in T lymphopoiesis." (2004). Electronic Theses and Dissertations. Paper 359.

https://doi.org/10.18297/etd/359

This Doctoral Dissertation is brought to you for free and open access by ThinkIR: The University of Louisville's Institutional Repository. It has been accepted for inclusion in Electronic Theses and Dissertations by an authorized administrator of ThinkIR: The University of Louisville's Institutional Repository. This title appears here courtesy of the author, who has retained all other copyrights. For more information, please contact thinkir@louisville.edu. 


\title{
THE TRANCRIPTIONAL REPRESSOR PROTEIN GROWTH FACTOR INDEPENDENCE-1B IN T LYMPHOPOIESIS
}

\author{
By \\ Loretta Doan \\ B.A. Indiana University Southeast, 1993 and 1999 \\ A Dissertation \\ Submitted to the Faculty of the \\ Graduate School of the University of Louisville \\ in Partial Fulfillment of the Requirements \\ for the Degree of
}

Doctor of Philosophy

Department of Biochemistry and Molecular Biology

University of Louisville

Louisville, Kentucky

May 2004 
THE TRANCRIPTIONAL REPRESSOR PROTEIN GROWTH FACTOR INDEPENDENCE-1B IN T LYMPHOPOIESIS

\author{
By \\ Loretta Doan \\ B.A. Indiana University Southeast, 1993 and 1999
}

A Dissertation approved on

December 12, 2003

By the following Dissertation Committee:

Dissertation Director 


\section{ACKNOWLEDGMENTS}

I would first like to thank my friend and mentor, Dr. H. Leighton Grimes, for all of his support and patience over the past few years. He has taught me much, not only of science, but of dedication and determination as well. He has always encouraged me to excel and to improve both professionally and personally. His guidance and example have provided me confidence and drive, and I am grateful for the opportunities he has afforded me. I would also like to thank the other members of my committee: Dr. Russell Prough, Dr. Carolyn Klinge, Dr. Barbara Clark, Dr. Michele Kosiewicz, and Dr. Tom Mitchell. Collectively, they have aided my scientific progress by challenging my understanding of my work and by keeping me focused. Additionally, each of them has contributed something unique to my experience through example, training, or individual conversations (or sometimes debates).

My research has benefited from the expertise of many collaborators outside of the University of Louisville. I would like to thank the following people whose work appears in this dissertation: Drs. Sabine Herblot and Trang Hoang, Drs. Qing Yu and Alfred Singer, Drs. C. Blake Gilks and Susan Porter, Drs. Marshall Horwitz and Zhijun Duan. Additionally, Dr. Howard T. Petrie was instrumental in the development of the new technique described in Chapter 2. 
I am grateful to all members of the faculty of the Department of Biochemistry and Molecular Biology for being knowledgeable, accessible, and genuinely interested in educating. The members of the Institute for Cellular Therapeutics have provided great support, scientifically and otherwise. Additionally, the members of the BMB staff, especially David Yates and Andrea Gregg, have helped immeasurably with bureaucratic red tape, which at times seemed never-ending. I also appreciate the camaraderie of all students, past and present, who have commiserated with my confusion or contributed to my growth. Or both.

Finally, I am forever indebted to my family for their love and continued support. My parents have been tremendous examples throughout my life, both personally and professionally. They have instilled in me the importance of a good work ethic, and have taught me a sense of responsibility. They have always encouraged me to pursue my (seemingly ever-changing) goals, and I know they are happy to see me accomplish this one. I would also like to thank my sister, Pauletta, without whose support I most likely would not have survived my undergraduate education in chemistry and my first year of graduate school. I am fortunate to have had them near during this time. 


\begin{abstract}
$\mathrm{T}$ lymphopoiesis has been an intense focus of immunological research since the discovery of the major histocompatibility complex (MHC) and T cell mediated transplant rejection. Additionally, researchers have long appreciated the role of $\mathrm{T}$ cells, and their development, in autoimmune disorders. Interest in this field has increased upon the realization that many leukemic oncoproteins are the very factors that control normal $\mathrm{T}$ cell development. Thus, the transcriptional networks that drive the function and development of $\mathrm{T}$ lymphocytes are closely linked with disease states.
\end{abstract}

Growth Factor Independence 1 (GFI1) and GFI1B are two very similar transcriptional repressor oncoproteins that are encoded by two different genes. Though nearly identical in their DNA binding and repressor domains, GFI1 and GFI1B are differentially expressed in normal tissues and in tumors of lymphoid lineage. GFI1 is frequently activated in mouse T cell leukemias, whereas GFI1B has not been found in T cell tumors. The work described in this dissertation provides insight into this phenomenon by delineating functional differences for GFI1 and GFI1B in T cells.

The first set of experiments compares the phenotypes engendered by transgenic expression of either GFI1 or GFI1B in developing and mature T lymphocytes. These analyses revealed that GFI1 enhances the response to T cell activation, whereas GFI1B decreases this response. Furthermore, transgenic GFI1B causes defects in thymocyte 
development, some of which result from a lack of survival signals. These defects can be corrected by transgenic expression of either BCL2, an inhibitor of apoptosis, or GFI1, suggesting that GFIl and GFI1B play opposing roles in T cell survival.

At least part of the effect of transgenic GFI1B results from GFI1B-mediated repression of Gfil transcription. We show that the transcription of Gfil is repressed in T cells by both GFI1 and GFI1B and that this is the result of direct binding to evolutionarily conserved GFI1/GFI1B recognition sequences in the Gfil promoter. Furthermore, we provide evidence that endogenous GFI1 regulates its own promoter in T cells, but not in a myeloid lineage cell line.

Finally, a more detailed analysis of the effect of GFI1B in T lymphopoiesis confirms a role for GFI1B in the survival and differentiation of thymocytes. This analysis revealed that transgenic expression of GFI1B results in altered expression of several members of $\mathrm{T}$ cell receptor (TCR) signaling pathways that are largely responsible for the survival and differentiation of thymocytes. The activity of the downstream effectors of these pathways appears to be decreased, providing mechanistic insight in to the function of GFI1B in T cells.

Our work is the first to describe a role for GFIB in T cells. We demonstrate that GFI1B negatively regulates the cellular response to activation through the TCR complex and provide preliminary evidence of a mechanism by which GFI1B mediates these effects. Furthermore, we delineate functional differences for GFI1 and GFI1B in T cells, providing insight into the differential expression of these two transcription factors. 


\section{TABLE OF CONTENTS}

PAGE

ACKNOWLEDGMENTS

ABSTRACT

LIST OF FIGURES

iii

iv

ix

CHAPTER

I. INTRODUCTION 1

A. Hematopoiesis, the immune system, and $\mathrm{T}$ cell development

B. GFI1 and GFI1B 11

C. Specific background and research objectives 16

II. INTRANUCLEAR STAINING OF PROTEINS IN HETEROGENEOUS CELL POPULATIONS AND VERIFICATION OF NUCLEAR LOCALIZATION BY FLOW CYTOMETRIC ANALYSIS

A. Introduction

B. Materials and methods $\quad 21$

C. Results and discussion

III. GFI1B EXPRESSION LEADS TO DEFECTS IN T-CELL ACTIVATION, IL-7R $\alpha$ EXPRESSION AND T-CELL LINEAGE COMMITMENT

A. Introduction 32

B. Materials and methods 34

C. Results 39

$\begin{array}{ll}\text { D. Discussion } & 70\end{array}$ 
IV. GFI1 IS AUTOREGULATORY IN T CELLS, BUT NOT IN A MYELOID LINEAGE CELL LINE

$\begin{array}{ll}\text { A. Introduction } & 75\end{array}$

B. Materials and methods $\quad 77$

$\begin{array}{ll}\text { C. Results } & 80\end{array}$

$\begin{array}{ll}\text { D. Discussion } & 109\end{array}$

V. GFI1B INTERFERES WITH POSITIVE SELECTION OF TCR TRANSGENIC THYMOCYTES

$\begin{array}{ll}\text { A. Introduction } & 113\end{array}$

B. Materials and methods 116

$\begin{array}{ll}\text { C. Results and discussion } & 117\end{array}$

VI. CLOSING REMARKS 135

$\begin{array}{ll}\text { REFERENCES } & 149\end{array}$

$\begin{array}{ll}\text { APPENDIX } & 164\end{array}$

$\begin{array}{ll}\text { CURRICULUM VITAE } & 166\end{array}$ 


\section{LIST OF FIGURES}

FIGURE

1. T Cell Receptor (TCR)-Major Histocompatibility Complex (MHC) interaction

2. The stages of T lymphopoiesis $\quad 8$

3. GFI1 and GFI1B are similar transcriptional repressors 14

4. Intracellular/intranuclear staining of fixed thymocytes 27

5. Retention of nuclear stains upon removal of cytoplasm and cellular membrane

6. Expression of endogenous GFI1 and GFI1B

7. Expression of transgenic GFI1 and GFI1B 45

8. GFI1 potentiates, whereas GFI1B inhibits, T cell activation 48

9. The GFI1B-induced inhibition of activation is cell autonomous and includes defective upregulation of early activation markers

10. Transgenic expression of GFI1B in the thymus results in enhanced numbers of mature CD4+ thymocytes and decreased CD8+ populations

11. Expression of GFI1B inhibits the development of female HY transgenic CD8 SP T cells

12. Expression of GFI1B overcomes a block to development in male HY transgenic $\mathrm{T}$ cells

13. GFI1B impairs co-receptor reversal

14. Transgenic BCL2 restores CD8 SP thymocytes in GFI1B transgenic mice

15. Transgenic GFI1 increases the generation of CD8 SP thymocytes in GFI1B transgenic mice 
16. Endogenous murine Gfil is repressed by GFI1 and GFI1B in thymocytes

17. Characterization of rat $G f i l$ locus and identification of the transcription start site

18. The rat Gfil promoter is highly homolgous to mouse and human loci, and is sensitive to repression by GFI1 and GFI1B

19. GFI1 and GFI1B repress endogenous human Gfil in the Jurkat T-ALL cell line

20. Formation of protein-DNA complexes with Jurkat nuclear extract and oligonucleotide probes containing GFIl consensus binding sites in rat Gfil promoter sequence

21. GFI1 and GFI1B bind in vitro to a conserved site in the rat Gfil promoter

22. Jurkat $\mathrm{T}$ cell nuclear extracts contain multiple GFI1 complexes with specific DNA sequence requirements and varying DNA binding affinities

23. GFI1 autoregulation occurs in T cells but not in a myeloid-lineage cell line

24. Transgenic GFI1B decreases the number of CD4 SP cells in Class II restricted TCR transgenic mice

25. GFI1B transgenic CD4 SP thymocytes display abnormal levels of maturation markers

26. CD4 SP cells of TCR GFI1B bi-transgenic mice do not mature normally

27. Schematic representation of major effectors in TCR signaling pathways

28. GFI1B transgenic thymocytes display abnormal expression of transcripts relevant to TCR signaling pathways

29. Transgenic GFI1B decreases DNA binding by TCR downstream effectors in bulk thymocytes

30. Schematic representation of GFI1 and GFI1B in positive selection of DP thymocytes 


\section{CHAPTER I}

\section{INTRODUCTION, BACKGROUND, AND RESEARCH OBJECTIVES}

\section{Hematopoiesis, the immune system, and $T$ cell development.}

Hematopoiesis-the process by which new blood cells are generated from selfrenewing pluripotent stem cells residing in the bone marrow-consists of two major arms, myelopoiesis and lymphopoiesis. Cells that are generated in the myelopoietic process include erythrocytes, which carry oxygen and carbon dioxide through the blood stream, and megakaryocytes, which generate platelets, cells that are necessary for blood clotting at the sites of injury. The remaining myeloid cells, along with all the cells of lymphoid lineage, make up the immune system. Immune cells protect the body from invasion and disease.

There are two basic classes of immune response—innate immunity and adaptive immunity - and each component of the immune system contributes to one or the other. Innate immunity is the body's first response, occurring within hours of invasion. This response is not pathogen-specific, and is carried out by several types of cells as well as complement factors. Epithelial cells function in innate immunity by forming physical barriers against pathogens. Phagocytes (a class of myeloid cells consisting of macrophages and neutrophils) act in the innate immune response by engulfing and destroying invading pathogens. The lymphoid cells that participate in innate immunity are natural killer (NK) cells. NK cells are programmed to destroy any non-self cell with 
which they come into contact. NK cells discriminate between self and non-self cells by recognition of self-MHC Class I on the surface of other cells. (Major Histocompatibility Complex, MHC, is discussed in more detail below.) This recognition signals the NK cell to suppress the killing machinery, thereby preventing the destruction of self cells.

The second line of defense, adaptive immunity, occurs over a period of days, and is pathogen-specific. In this process, the immune system recognizes and adapts to the presence of invading pathogens, resulting in the specific elimination of either invading organisms or of infected host cells. Furthermore, the adaptive immune system has memory. That is, after the initial infection by an invading organism has been cleared by the adaptive immune system, re-introduction of the same pathogen results in a more rapid and efficient response. Adaptive immunity comes in two forms, humoral immunity and cell-mediated immunity, which are executed by B and T lymphocytes, respectively. B cells mediate humoral immunity by secreting antibodies that recognize and bind to pathogens, resulting in the destruction of the invader by phagocytes.

Cell-mediated immunity is controlled by T cells, which become activated against pathogens by interaction of the membrane-bound T cell receptor (TCR) complex and pathogenic antigens presented on the surface of other host cells in the context of self major histocompatibility complex (MHC) molecules. MHC molecules are present on the surface of all cells within the body and, under normal physiological conditions, present self-antigens to TCRs on mature T cells. This constant low-level interaction contributes to the maintenance and survival of a diverse $\mathrm{T}$ cell population, a process known as $\mathrm{T}$ cell homeostasis. However, upon cellular infection or phagocytosis of an invading organism, pathogen-specific proteins are processed to produce peptide antigens, which are bound by 
MHC and presented on the cell's surface. It is this antigen/MHC complex that is recognized by the pathogen-specific TCR on circulating T cells, initiating and sustaining a cellular immune response. (See Figure 1.)

There are two types of $\mathrm{T}$ lymphocytes, helper $\mathrm{T}$ cells and cytotoxic $\mathrm{T}$ cells. $\mathrm{T}$ helper cells are activated by antigen in the context of MHC Class II, which is expressed almost exclusively by professional antigen presenting cells. Once activated, a $\mathrm{T}$ helper cell proliferates (a process known as clonal expansion), promotes the proliferation of B cells, activates macrophages, and promotes inflammation, all of which can contribute to the destruction of the invader. Cytolytic T cells are activated by antigen in the context of MHC Class I molecules on the surface of infected cells, resulting in the specific lysis of the infected target cell by the T cell. All cells express MHC Class I, because any cell in the body is a potential target for infection.

All $\mathrm{T}$ cells can be experimentally identified by surface expression of the $\mathrm{T}$ cell receptor (TCR) complex. Furthermore, T helper cells are easily identified by the surface expression of the CD4 co-receptor molecule, and cytotoxic T cells are characterized by the expression of the CD8 co-receptor. The TCR complex is responsible for antigen recognition and initiation of intracellular activation signals, the latter being largely regulated through a family of surface proteins known as the CD3 complex. Both CD4 and CD8 co-receptors assist in the initiation of signaling cascades, and no qualitative difference between the signaling events mediated by the two co-receptors has been defined, leaving a mystery as to the mechanisms governing, and the reasons for, the specificity of expression of these two co-receptors on the two classes of T cells. 


\section{Figure 1. T Cell Receptor (TCR)-Major Histocompatibility Complex (MHC)}

interaction. Schematic representation of a TCR recognizing and binding to peptide presented by MHC. $C \beta$ and $V \beta$, respectively, are the constant and variable regions of the TCR $\beta$ chain. The peptide antigen is bound in the groove between the $\alpha$ and $\beta$ chains of the MHC molecule, and is shown as a rectangle. 
Figure 1

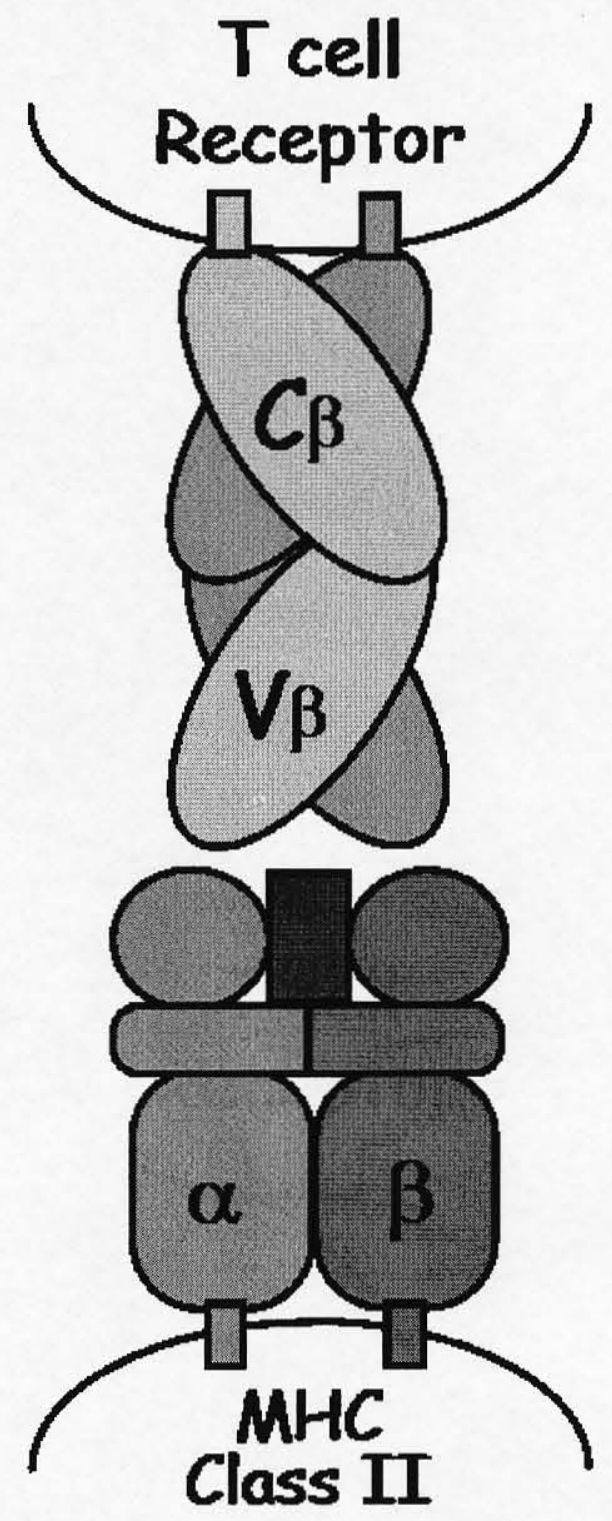

- 5 - 
Antigen specificity and affinity of the TCR complex are dictated by the sequences of the TCR $\alpha$ and $\beta$ chains. Diverse sequences are generated by chromosomal rearrangement of the variable regions of these genes, which is mediated by the products of Recombinase Activating Genes (RAG) 1 and 2, and cannot occur in the absence of either RAG1 or RAG2. Furthermore, surface expression of one successfully rearranged TCR $\alpha$ or $\beta$ chain results in signaling that suppresses further rearrangement on the other allele, a process known as allelic exclusion. Allelic exclusion results in the expression of only one TCR on most T cells, ensuring that each cell has specific affinity for antigen and MHC, providing the required diversity of the cellular immune system. In addition to controlling the function of mature $\mathrm{T}$ cells in immune responses, the affinity/avidity of TCR/MHC interactions also determine the fate of developing T cells in the thymus, a process known as $\mathrm{T}$ lymphopoiesis (recently reviewed in $(1 ; 2))$.

$\mathrm{T}$ lymphopoiesis begins when $\mathrm{T}$ cell precursors, prior to committing to the $\mathrm{T}$ lineage, leave the bone marrow and enter the thymus. The development of thymocytes is tracked and partially defined by the cell-surface expression of the CD4 and CD8 coreceptors (see Figure 2). The earliest thymocytes express neither CD4 nor CD8 and are referred to as double negative (DN) cells. After commitment to the T lineage, DN thymocytes express a pre-TCR, which is a heterodimer consisting of the pre-T $\alpha$ and a rearranged TCR $\beta$ chain. Signals from the pre-TCR are required for further development and differentiation into the next stage of $T$ lymphopoiesis, the double positive (DP) stage, where both CD4 and CD8 are expressed on the cell surface. Rearrangement of the TCR $\alpha$ chain occurs upon differentiation into the DP stage. Once expressed, the TCR $\alpha$ chain replaces pre-T $\alpha$ and provides the final requirement for a mature TCR complex, signals 
Figure 2. The stages of $T$ lymphopoiesis. A. Schematic representation of the $T$ lymphopoietic process. The most primitive thymocytes express neither CD4 nor CD8 (double negative, DN). This stage can be further sub-classified based on the expression of surface markers CD44 and CD25. Survival and differentiation during the DN stages are dependent upon signals from cytokines, such as IL-7, and from the pre-TCR. In the next stage of differentiation, cells express both CD4 and CD8 simultaneously (double positive, DP), and the TCR replaces the pre-TCR. These early stages of T lymphopoiesis occur in the cortex of the thymus. Finally, thymocytes down-regulate either CD4 or CD8 to become $\mathrm{CD} 4^{+}$or $\mathrm{CD} 8^{+}$single positive (SP), translocate into the medulla and undergo final maturation. This lineage commitment depends on signals from the TCR. B. Flow cytometric analysis of CD4 and CD8 expression on C57/B16 mouse thymocytes. The subpopulations are gated and identified. 
Figure 2

A

Cortex Medulla

TCR

Pre-TCR

\section{DN}

T cell

Prog.

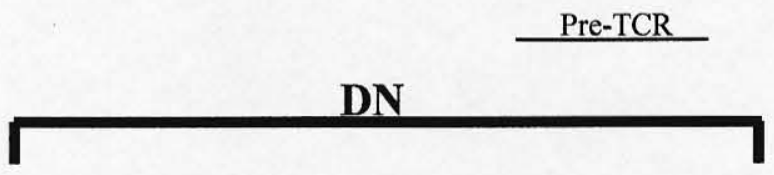

$\mathrm{CD4}^{+}$

CD8

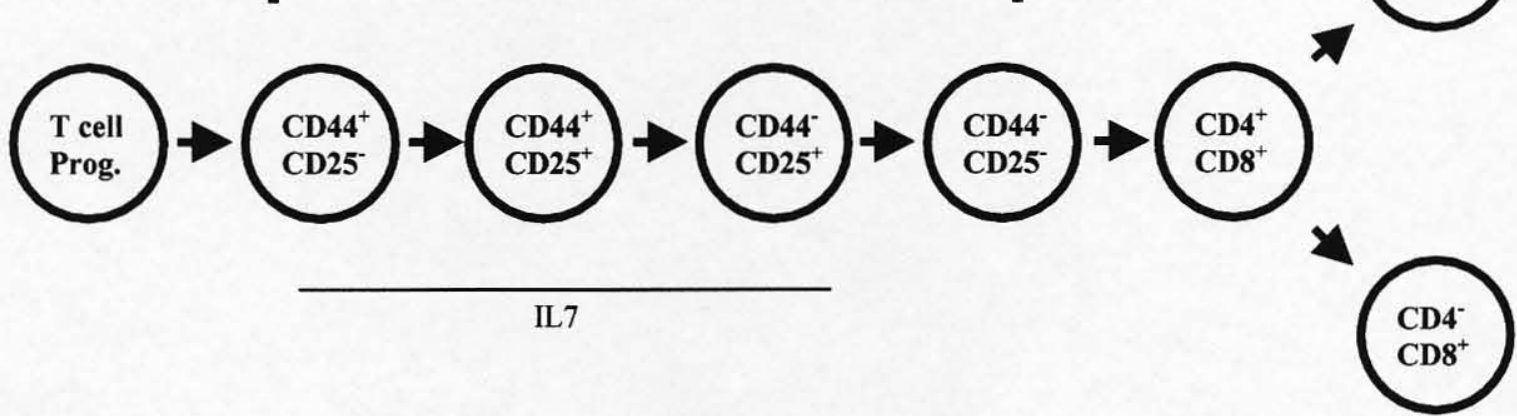

B

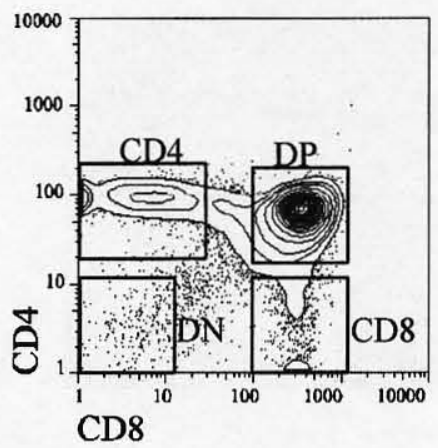


from which are necessary for further development. The final stage of thymocyte maturation requires down-regulation of one or the other co-receptors and results in differentiation into either CD4 (helper) or CD8 (cytolytic) single positive (SP) T cells. This stage of maturation requires the integration of two distinct processes-positive selection, a process by which a cell receives survival signals; and lineage commitment, a process by which a cell makes fate decisions that result in the expression of a single coreceptor. Neither process has been fully defined, though extensive research is ongoing in both areas. However, positive selection is absolutely dependent upon appropriate TCRmediated signals. In the thymus, as in peripheral T cell homeostasis, TCR signaling events are initiated by interaction of the TCR with self-peptide presented by MHC on the surface of thymic antigen presenting cells. It is the affinity of this interaction that determines whether the maturing $\mathrm{T}$ cell survives or dies.

Signals from both cytokine receptors and the TCR are required for survival and positive selection. Death of developing thymocytes occurs as a result of either very weak or very strong TCR signals. An insufficient signal results in apoptotic death that is referred to as "death by neglect", while a very strong signal results in apoptotic death that is known as negative selection. Death by neglect assures that incompetent $\mathrm{T}$ cells are not released into the immune system, while negative selection assures that autoreactive $\mathrm{T}$ cells do not escape into the periphery and is vital to the prevention of autoimmune disease.

Survival of developing $\mathrm{T}$ cells is dependent upon an intermediate strength of signal from the TCR. A thymocyte, in the course of development and migration through the thymus, has contact with many different antigen presenting cells (APCs), each 
presenting self-peptide/MHC complexes for interaction with the TCR. Furthermore, cytokines, expressed at different anatomical locations within the thymus, interact with their corresponding receptors on the surface of developing thymocytes to provide survival signals. For example, deletion of IL7R $\alpha$ results in drastically reduced thymic cellularity resulting from a severe block to development at the DN stage (3). Thus, a combination of TCR signaling and cytokine signaling is critical for T lymphopoiesis.

Lineage commitment is closely linked to the MHC-specificity of the TCR; typically, CD4 SP T cells are restricted on MHC Class II molecules, while CD8 SP T cells are restricted on MHC Class I molecules. Furthermore, the MCH loci are comprised of several different genes that are highly polymorphic, and it is these polymorphisms that form the basis of self and non-self specificity of recognition by $\mathrm{T}$ cells. In humans and outbred animals, the two different alleles of MHC genes, both of which are expressed, encode distinct polymorphic proteins, resulting in even greater diversity. Each allele is designated as a specific haplotype, since it comprises only half of the genetic and proteomic information. On the other hand, in inbred laboratory mouse strains, both alleles of the MHC locus encode the same polymorphisms, resulting in the expression of only one protein for each MHC gene. Therefore, every gene in the MHC locus of these mice has the same haplotype designation, which is a small letter of the alphabet (some common haplotypes are $b, d$, and $k$ ). Many $T$ cells from a mouse with a particular haplotype, by virtue of TCR-MHC interaction, recognize as foreign MHC molecules of any other haplotype. This recognition forms the basis for transplant rejection and, indeed, was the early discovery that led to the intense interest that has developed in the area of T lymphopoiesis. 
While much is understood about phenotypic and cell-surface changes that accompany $\mathrm{T}$ lymphopoiesis, and quite a bit has been discovered about early signaling events in TCR-mediated activation, a minimal understanding exists of the transcriptional network that links the early signaling events to the phenotypic changes. Several transcription factors have been implicated in the differentiation of thymocytes, including EGR-1, Notch1 (a cell-surface receptor that is cleaved upon ligand binding to form intracellular Notch (ICN), a transcriptional regulator), and the E2A gene products, E12 and E47 (4-6). Additionally, the activity of certain transcription factors has been shown to increase upon TCR activation, including that of NFAT, NFkB, and AP-1, each under the control of distinct second messengers and/or signaling pathways (recently reviewed in (7)). Furthermore, different arms of the mitogen activated protein kinase (MAPK) pathway have been shown to preferentially activate during either positive or negative selection, providing some insight into how the activation of the same TCR can lead to dichotomous outcomes (recently reviewed in (1)). However, an understanding of the integration of activation, survival and differentiation signals, which is the key to understanding the continuous process of $\mathrm{T}$ lymphopoiesis, has remained elusive.

\section{Gfil and GfilB.}

The Growth Factor Independence-1 (GFI1) and GFI1B proteins are closely related nuclear oncoproteins that may regulate cytokine pathways. Gfil was originally identified as the gene upregulated by insertion of Moloney murine leukemia virus (MoMLV) in a thymic lymphoma that was selected for its ability to grow in the absence of the T-cell cytokine IL-2 (8). GFI1 is mildly anti-apoptotic and inhibits growth arrest 
of IL-2 dependent $T$ cell lines under conditions of limiting IL-2 (8;9). Furthermore, forced expression of Gfil potentiates the outgrowth of cell lines that do not depend on the addition of IL-2 to the media, without inducing production of IL-2 within the culture (8). In fact, $2780 \mathrm{a}$ cells that were infected with $G f i 1$-encoding retrovirus escaped both apoptosis and G1 arrest induced by the withdrawal of IL-2 (9). GfilB was identified by low stringency hybridization screening with a cDNA probe encoding the zinc-finger region of Gfil (10). GFI1B inhibits both growth arrest and IL-6-induced differentiation of M1 myelomonocytic cells (10).

GFI1 and GFI1B are 97\% homologous in the carboxy-terminal 165 amino acids that encode six Cys-His zinc fingers. An amino-terminal 20-amino-acid SNAG domain (found in the Snail and GFI1 family of proteins), which is responsible for nuclear localization and transcriptional repressor function, is also highly conserved $(9 ; 10)$ (see Figure 3). In fact, a single mutation in the SNAG domain of the Proline at position 2 to Alanine (P2A) is sufficient to abrogate repression activity in transient transcription assays, without affecting either nuclear localization or DNA binding capability (9). Additionally, 2780a cells infected with P2A-GFI1-encoding retrovirus were unable to escape G1 arrest in the absence of IL-2, suggesting that the oncogenic function of GFI1 is dependent upon its transcriptional repressor activity (9).

Consensus DNA recognition sequences for GFI1 and GFI1B have been defined using bacterially synthesized proteins encoding GST fused to GFI1 or GFI1B. Under the conditions used to define these sites, GFI1 and GFI1B bind to virtually identical DNA consensus sequences, defined as 5'-TAAATCAC(A/T)GCA-3' for GFI1 and 
Figure 3. GFI1 and GFI1B are similar transcriptional repressors. Gfil and $G f i 1 B$ are $97 \%$ homologous in the region encoding the $\mathrm{Zn}$ fingers and the proteins recognize the same core DNA sequence. The 20-amino-acid SNAG domain of GFI1B differs from that of GFI1 by a single amino acid. Target genes proposed in the literature are listed (10-14) 
Figure 3

\section{GFI1}

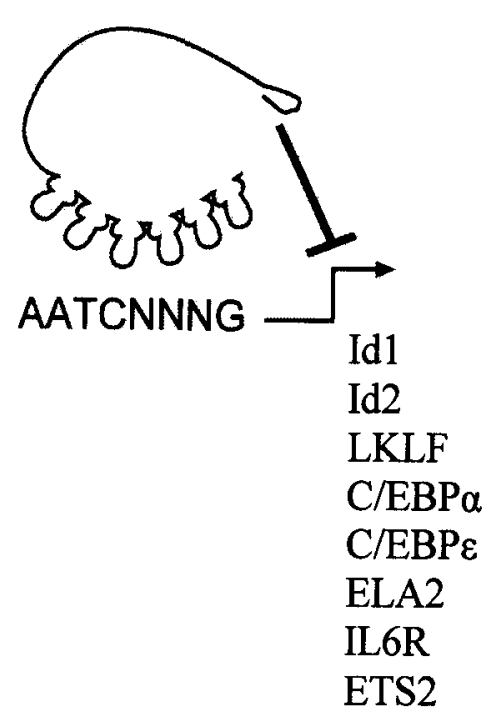

GFI1B

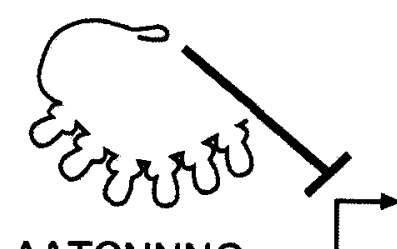

AAICNNNG $-\mathrm{P} 21^{\text {WAF1 }}$

Gfil

Socs1

Socs3

ETS2 
5'-TAAATCACTGC(A/T)-3' for GFI1B $(10 ; 15)$. The AATC core was observed in $100 \%$ of the sequences that were bound by either GFI1 or GFI1B zinc fingers.

Several GFI1 targets genes have been suggested by virtue of being up-regulated in $\mathrm{Gfil}^{-/}$mice. These include TNFR-associated factor (TRAF) 5, Lung Kruppel Like Factor (LKLF), and Id1 and Id2, all of which have been shown to play roles in T lymphopoiesis (11). Additional potential targets for GFI1 repression include C/EBP $\alpha$ and PU.1, both of which are required for proper development of neutrophils (16). Other putative targets have been identified by chromatin immunoprecipitation (ChIP) analysis, and include E2F family members E2F5 and E2F6, and Ets family members Ets2 and cMyc. Furthermore, this analysis identified binding by GFI1 to the GfilB promoter in three different cell lines (13). Suggested targets for GFI1B have also been identified and include suppressor of cytokine signaling (SOCS) 1 and 3 (14) and p21 (10).

Despite the extensive similarities of GFI1 and GFI1B, each contains a region unique in amino acid sequence between the SNAG domain and the $\mathrm{Zn}$ fingers, the activities of which are yet to be defined. These unique regions may be the key to the distinct physiological roles of these two very similar transcription factors.

Gene deletion studies have shown that $G f i l$ and $G f i l B$ play distinct, pivotal roles in hematopoiesis. Gfil-deficient mice display both thymic and peripheral T lymphopenia, with severe abnormalities in pre-T cell development $(11 ; 16 ; 17)$. Furthermore, they display a profound defect in neutrophil differentiation, which leads to neutropenia $(16 ; 17)$. In support of this apparent role in neutrophil differentiation, several putative targets of GFI1 have been identified, including $E L A 2, A z u, A A T$, and $A C T$, all of which are granulocyte specific genes (13). Additionally, humans have been identified 
that harbor a single allelic mutation of $G f i 1$, presumably resulting in the expression of a dominant negative protein along with the wild type GFI1 (Gfi $\left.1^{d n / w t}\right)$. These mutations were identified in patients with specific types of neutropenia, a phenotype which mimics that seen in $G f i 1^{1 /}$ mice. However, unlike Gfil-deficient mice, which have severe T lymphoid abnormalities, the $G f I^{d n / w t}$ patients display only mild disruptions in T cell populations.

While Gfil is essential to proper development of lymphoid and granulocytic cells, gene deletion studies in mice revealed that $G f i 1 B$ is necessary for the development of megakaryocytes and for definitive erythropoiesis (18). Deficiency of GfilB is embryonic lethal by day E15 (18). In accordance with the hematopoietic phenotypes of Gfil and $G f i 1 B$ deficiency, the thymus of adult rats expresses predominantly $G f i 1$, with $G f i 1 B$ being undetectable in Northern analysis of whole thymus. GfilB is the predominant factor expressed in spleen, the major site of erythropoiesis in adults. Both factors are expressed in bone marrow, where many types of hematopoietic cells reside, including hematopoietic stem cells (10).

\section{Specific background and research objectives.}

It has been reported that in T cells, transgenic expression of GFIl causes an increase in the response to TCR-stimulated activation (19-21). In addition, Gfil transcription is upregulated upon TCR-mediated activation of mature T cells $(8 ; 21)$. Despite the fact that T lymphopoiesis is driven largely by TCR signals, and that other genes that are downstream of TCR-mediated activation, such as EGR-1, have been shown to be instrumental in the development of T cells (4), the expression pattern and role of 
GFI1 in developing thymocytes have not been examined. Furthermore, the degree of sequence homology between GFI1 and GFI1B, along with the similar biochemical and oncogenic functions of these two factors, suggest the possibility that GFI1B, if expressed in T cells, may perform functions similar to those of GFI1.

To further examine the function of GFI1 in T cells and developing thymocytes, and to begin to determine whether GFI1B functions in a similar manner, transgenic mice were created that express either GFI1 or GFI1B in developing thymocytes and in mature T cells. Examination of these GFI1- and GFI1B-transgenic mice in this laboratory confirmed that, as previously published, forced expression of GFI1 potentiates $\mathrm{T}$ cell activation in vitro. However, GFI1B-transgenic cells responded poorly to TCR activation, a result directly opposite to that observed in GFI1-transgenic cells. Furthermore, though expression of transgenic GIF1 produces no gross thymic phenotype, expression of transgenic GFI1B results in anomalies in T cell development. Specifically, GFI1B-transgenic mice produce large numbers of CD4 SP thymocytes with phenotypic features of maturity, and very few CD8 SP thymocytes.

My goals for this project were three-fold. First, I sought to determine the physiological relevance of the observed effects of GFI1B on TCR activation in T lymphopoiesis by examining expression of endogenous GFI1B in developing thymocytes. Though GfilB could not be detected by Northern analysis of whole rat thymus, the possibility remained that this factor is expressed in certain subsets of thymocytes. For example, the DN cells, of which there are four distinct subpopulations, make up only about $1-3 \%$ of total thymocytes. Therefore, if GFI1B were expressed in only a subset of DN thymocytes, it would be virtually impossible to detect it by 
previously utilized methods. However, developmental-specific expression of a factor that negatively regulates TCR signaling could be instrumental in controlling the survival and differentiation of cells. Secondly, I sought to determine the specific T lymphopoietic process(es) that may be affected in GFI1B-transgenic mice. Because selection of thymocytes is central to their development, I focused on determining whether GFI1Btransgenic thymocytes are defective in positive selection, negative selection, or both. Finally, I wanted to identify downstream targets of GFI1 and GFI1B that may mediate the observed effects on activation. Several downstream effectors of TCR stimulation are well defined, and the interruption or propagation of any of these pathways could result in the observed effects on activation. I therefore sought to determine whether GFI1B transgenic thymocytes display altered expression levels of members of these signaling pathways.

My second project arose as a result of an observation made regarding the GFI1and GFI1B-transgenic mice. Upon Northern analysis of RNA from transgenic thymocytes, it was observed that in both GFI1- and GFI1B-transgenic animals there was a reduction in endogenous Gfil message. This presented the possibility that GFI1 and GFI1B may negatively regulate the expression of Gfil. My goals in this project were to determine whether the observed repression is direct and whether it is conserved among species and cell types. 


\section{CHAPTER II}

\section{INTRANUCLEAR STAINING OF PROTEINS IN HETEROGENEOUS CELL POPULATIONS AND VERIFICATION OF NUCLEAR LOCALIZATION BY FLOW CYTOMETRIC ANALYSIS}

\section{Introduction.}

Traditionally, cells and tissues have been tested for the presence of specific proteins by biochemical methods such as Western blotting. While this method has advantages, such as the determination of apparent molecular weight and oligomerization status, it is rather time-consuming. Furthermore, if a protein is expressed at very low levels, or in only a fraction of cells in a heterogeneous cell population, Western blotting can be unreliable, providing false negative results. For example, Western blot analysis failed to detect the transcription factor Growth Factor Independence-1B (GFI1B) in wild type thymocytes, even though its expression can be confirmed by RT-PCR. It is often possible to detect nuclear transcription factors by immunofluorescence microscopy, advantages of which include visual interpretation of data in intact cells, allowing the determination of not only nuclear vs. cytoplasmic localization, but of subnuclear localization patterns as well. However, it is difficult to detect poorly expressed proteins with low affinity antibodies by this method, due in part to limitations of the human eye. While it is possible to enhance the signal using sophisticated software packages, this 
requires a skilled and knowledgeable microscopist. We therefore turned to flow cytometry to detect GFI1B in thymocyte subsets.

Flow cytometry is a method whereby the expression of specific molecules can be assessed on the single-cell level by using antibodies against the proteins of interest. The protein-specific antibodies are conjugated to fluorochromes, and as the cells pass through the cytometer, they are exposed to different wavelengths of light to excite the fluorochromes, which subsequently emit light of a specific wavelength that is detected by the instrument. Currently, the industry standard flow cytometers allow the simultaneous detection and separation of four fluorochromes, making it possible to examine the expression of up to four different proteins on a cell. Traditionally, flow cytometry has been used to examine cell-surface proteins; however, methods have been developed to allow the use of this method for examination of intracellular proteins. This often involves first staining the cells with antibodies against cell-surface molecules in order to be able to electronically isolate subsets of cell populations. Nonetheless, as in microscopy, it is difficult to detect poorly expressed intracellular/intranuclear proteins (such as GFI1B) using this method because of the background produced upon fixation of cells after surface staining. We tested several methods of fixing and permeabilizing cells $(22 ; 23)$ with limited success before finding and adapting the method described here. These procedures are readily amenable to the detection of other nuclear transcription factors.

We have adapted flow cytometric methods, which can be easily performed by lab personnel with minimum working knowledge of benchtop flow cytometry instruments, to detect nuclear proteins using polyclonal antibodies. We have used standard techniques 
for staining the surface of cells, and have modified techniques for fixing the surface stain and permeabilizing both the cellular and nuclear membranes. Additionally, we have adapted a method for isolating nuclei (24) in order to determine whether a stain is nuclear or cytoplasmic. Together, these techniques provide a fast, easy alternative to classical methods for the detection of intracellular proteins and the determination of subcellular localization.

\section{Materials and methods.}

Mice and preparation of cell suspensions. Mice were housed in the Baxter Barrier facility at the University of Louisville Medical School. All animal protocols were approved by the University of Louisville Institutional Animal Care and Use Committee (IACUC).

Thymi of 4-8-week-old mice were removed into Hank's Balanced Saline Solution plus $2 \%$ heat inactivated fetal bovine serum (HBSS $\triangle \mathrm{FBS}$ ) and disrupted between the ends of glass slides. The cell suspension was passed through Nytex mesh to remove debris, and sedimented in a Sorvall 7 centrifuge at $1200 \mathrm{rpm}$. The cell pellet was washed by resuspension in $10 \mathrm{~mL}$ of HBSS $\triangle \mathrm{FBS}$ and sedimented as described. Cells were resuspended in $10 \mathrm{~mL}$ of HBSS $\triangle \mathrm{FBS}$ and counted using a Coulter Z2 Particle Counter. 1 x $10^{6}$ cells were aliquoted into pre-labeled Falcon 2008 tubes for each stain to be performed.

Surface staining. Surface proteins were detected using a standard protocol. Briefly, aliquoted cells were washed once with FACS $@$ media (HBSS with $0.1 \%$ BSA, $0.1 \%$ Sodium Azide, and $0.036 \%$ Sodium Bicarbonate), and spun as described. The 
supernatant was poured off, cells were resuspended in $100 \mu \mathrm{L}$ of FACS $\otimes$ media, and 20 $\mu \mathrm{L}$ of the appropriate antibody dilution was added (see below for antibody dilutions). The cells were incubated for 20 minutes at $4^{\circ} \mathrm{C}$ in the dark, washed once with FACS $\circledast$ media and placed on ice.

Fixing and intracellular/intranuclear staining. After surface staining and washing, cells were washed once with phosphate buffered saline without calcium or magnesium (PBS-), then resuspended in $100 \mu \mathrm{L}$ of PBS-. A vial of $16 \%$ formaldehyde (Polysciences catalog \#18814) was opened, and a 4\% solution was made by dilution into PBS-; the formaldehyde stock was discarded. $100 \mu \mathrm{L}$ of $4 \%$ formaldehyde was added to each tube of cells while gently vortexing. The cells were covered with aluminum foil and incubated on ice for 3 hours. Cells were washed twice with PBS-, and resuspended in $100 \mu \mathrm{L}$ of stain buffer (PBS- plus 5\% FBS and $0.5 \%$ TritonX-100). The cells were covered and incubated on ice for 30 minutes, after which time $20 \mu \mathrm{L}$ of the appropriate primary antibody dilution was added. The cells were gently mixed and incubated on ice for 30 minutes, covered. Cells were washed once with stain buffer, resuspended in 100 $\mu \mathrm{L}$ of stain buffer, and $20 \mu \mathrm{L}$ of secondary antibody was added. The cells were covered and incubated on ice for 30 minutes. Finally, cells were washed with stain buffer and resuspended in $250 \mu \mathrm{L}$ of stain buffer for data acquisition. All solutions were kept on ice. Permeabilization and staining of unfixed cells. Cells were aliquoted and washed with FACS $\circledast$ media as described for surface staining and resuspended in $100 \mu \mathrm{L}$ of FACS $\circledast$ media with $0.03 \%$ saponin (FACS plus saponin) (22). $20 \mu \mathrm{L}$ of the appropriate primary antibody was added to each tube, and the cells were incubated at $4^{\circ} \mathrm{C}$ in the dark for 30 minutes. Cells were washed once with FACS plus saponin, and resuspended in $100 \mu \mathrm{L}$ 
of FACS plus saponin. $20 \mu \mathrm{L}$ of the appropriate secondary antibody dilution was added, and the cells were incubated for 30 minutes at $4^{\circ} \mathrm{C}$ in the dark. Finally, cells were washed once with FACS plus saponin and once with FACS $\circledast$ media. The cells were resuspended in either $250 \mu \mathrm{L}$ of FACS $\otimes$ media or $250 \mu \mathrm{L}$ of nuclear isolation media (NIM; FACS $\circledast$ media plus $1 \mathrm{mM}$ EDTA and $0.5 \%$ NP-40). All wash steps were in $2 \mathrm{~mL}$ of media.

Antibody panels and dilutions and flow cytometry. Surface staining was performed using anti-CD4 (RM4-5)-APC and anti-CD8 (53-6.7)-PE, both from Pharmingen. Antibodies were diluted together into FACS $\otimes$ media, with a dilution factor of $1: 100$ for each antibody. For intracellular/intranuclear staining, antibodies were diluted using the permeabilization buffer appropriate for the method and were used at the following dilutions: goat polyclonal anti-GFI1 (sc-8558, Santa Cruz Biotechnology) at 1:10, goat polyclonal anti-GFI1B (sc-8559) at 1:50, and normal goat IgG (sc-2028) at 1:25 and 1:125 for GFI1 and GFI1B controls, respectively; rabbit polyclonal anti-EGR1 (sc-189) at 1:200 (23), and normal rabbit IgG control (sc-2027) at 1:400. Dilution factors for IgG control antibodies were based on the final $\mu \mathrm{g}$ quantity of the experimental antibody used. Secondary antibodies, bovine anti-goat FITC (sc-2348) and donkey anti-rabbit FITC (711-096-152, Jackson Laboratories), were used at a 1:100 dilution. Anti-BCL2-FITC and its FITC conjugated isotype control (catalog \#554221, BD Pharmingen) were used at a 1:33 dilution. Flow cytometry was performed on a Becton Dickinson FACSCaliburTM flow cytometer, and analysis was performed using FlowJoTM (TriStar) software.

Fluorescence Microscopy. Green fluorescence protein (GFP) transgenic mouse thymocytes were sorted to $>95 \%$ purity for positive GFP fluorescence on a FACS 
Vantage Cell Sorter (Becton Dickinson) using $488 \mathrm{~nm}$ excitation and standard FITC emission optics. 700,000 sorted thymocytes were stained with $5 \mathrm{uM}$ Hoechst 33342 in FACS $®$ media for 30 minutes at $37^{\circ} \mathrm{C}$. Stained cells were divided into two equal parts, pelleted, and resuspended in $100 \mu \mathrm{L}$ of FACS $\otimes$ media or NIM. A wet mount of $50 \mu \mathrm{L}$ of each sample was analyzed for GFP (cytoplasm) and Hoechst fluorescence (nuclei) on an Olympus IX50 fluorescence microscope.

\section{Results and discussion.}

\section{BCL2 and EGR1 confirm valid fixation of surface stain and permeabilization.}

We were interested in using flow cytometry to detect nuclear transcription factors that are expressed at low levels within thymocyte subsets. We started with commercially available antisera to the GFI1B transcription factor. Though unfixed GFI1B transgenic thymocytes showed GFI1B-positive staining when permeabilized with $0.03 \%$ saponin, we were unable to detect positive staining if cells had been fixed in a solution of $1 \%$ formaldehyde (Polysciences catalog \#04018) in HBSS despite attempts to permeabilize with $0.03 \%$ or $0.3 \%$ saponin, and $0.1 \%$ or $0.5 \%$ TritonX-100 (22;23). We therefore purchased an EM-grade formaldehyde (Polysciences catalog \#18814) that is supplied in individual $10-\mathrm{mL}$ aliquots. The formaldehyde was stored in the dark, and a vial was opened immediately prior to use. The unused portion was discarded, limiting the exposure of the reagent to light and to oxidizing substances in the air. By so limiting the degradation of the formaldehyde, we decreased the autofluorescence of the fixed cells (25), thereby increasing our signal-to-noise ratio and allowing detection of transcription factors in thymocyte subsets. 
To ensure the accuracy of our results, we examined proteins with established expression patterns and cellular localization. By confirming the known expression profile of BCL2 (26) (Figure 4), we were able to determine that our fixation and permeabilization methods do not disturb antigen-antibody interactions. Moreover, detection of the nuclear transcription factor EGR1 (27) using the same protocol served as a control for permeabilization of the nuclear membrane (Figure 4). In sum, our adaptation of established permeabilization and fixation techniques allows sensitive and accurate detection of intranuclear antigens without disrupting membrane or cytoplasmic staining.

GFI1B is not detectable in most thymocytes of wild-type mice. In fact, utilization of the sensitive methods described here allowed detection in only the relatively rare CD44 DN subsets (19). Therefore, wild-type thymocytes served as negative controls for staining with anti-GFI1B (data not shown), further confirming the specificity of our reagents and methods.

Isolation and analysis of nuclei confirm nuclear localization of transcription factors.

For our experiments it was necessary to confirm nuclear localization of our transgenic transcription factors. To accomplish this task, we adapted a method that has historically been used to study nuclei for DNA content (24).

Unfixed cells were permeabilized and stained as described, then resuspended in either FACS $\circledast$ media or NIM for the isolation of nuclei. The removal of the cytoplasm was confirmed separately by microscopic analysis of sorted GFP-positive thymocytes that had been stained with the DNA dye Hoechst 33342 and resuspended in either FACS or NIM (Figure 5), and for stained cells by a change in forward and side scatter properties 
Figure 4. Intracellular/intranuclear staining of fixed thymocytes. Shown is a representative FACS plot of surface-stained, fixed, permeabilized and intracellularly stained thymocytes. Gates are drawn around each of the four major subsets, and are labeled as DN (double negative), DP (double positive), CD8 (CD8 single positive), and CD4 (CD4 single positive). The expression of BCL2 and EGR1 was examined by intracellular/intranuclear staining and the results are shown in histogram format. In each histogram overlay, the dotted line represents staining with the control $\operatorname{IgG}$ and the solid line represents the experimental stain. As expected, BCL2 is expressed highly in CD4, CD8, and most DN cells, and at low levels in DP cells. A positive shift in fluorescence in the presence of the anti-EGR1 antibody occurred in all thymocyte subsets, though the range of expression level is larger in DN thymocytes as indicated by a broader histogram. 
Figure 4
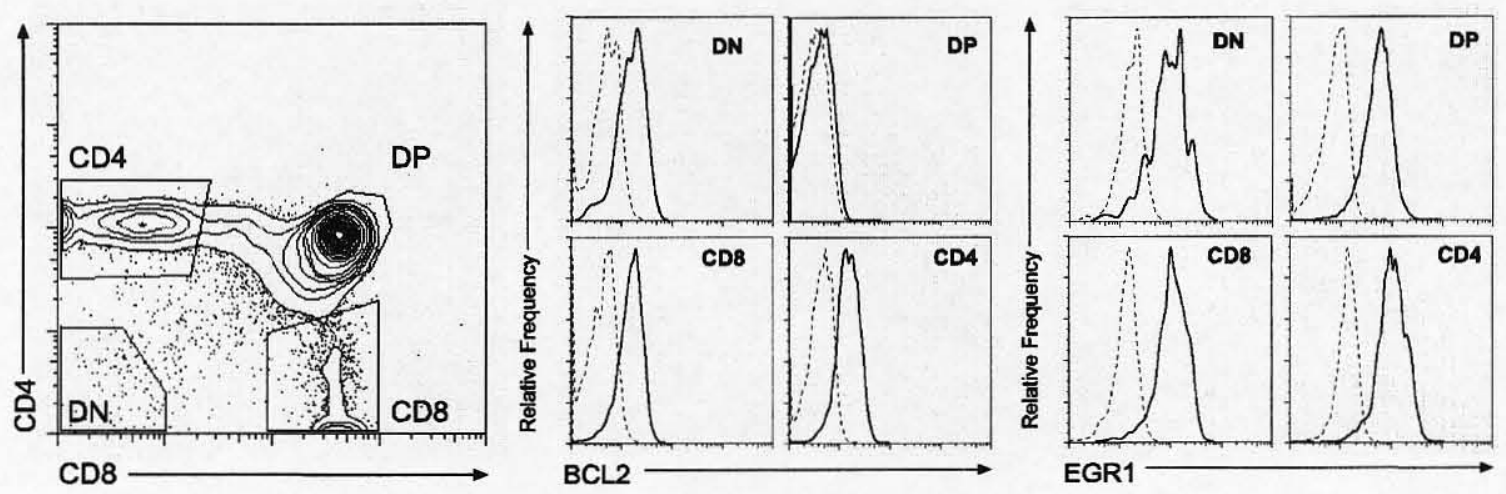
(data not shown). Permeabilized and stained cells and nuclei were analyzed by flow cytometry for a positive shift in fluorescence intensity. Figure 5 shows histogram plots of cells (top) versus nuclei (bottom) for BCL2, EGR1, GFI1B, and GFI1 (left to right). Each histogram overlay includes both the experimental stain (solid bold line) and the corresponding IgG control (dashed line). The stains for BCL2 and EGR1 were performed using wild type thymocytes, while those for GFI1B and GFI1 were performed using thymocytes from mice that were transgenic for GFI1B or GFI1, respectively. BCL2, which localizes to the mitochondria, was included as a cytoplasmic control. Total thymocytes (cells) show variable expression of BCL2, mirroring the expression profile in thymocyte subsets. However, isolated nuclei do not show any positive staining for $\mathrm{BCL} 2$, as the mitochondria are removed with the cytoplasm during the nuclear isolation step. Results are shown for EGR1, GFI1B, and GFI1. The intensity of signal for an intranuclear stain depends on both the affinity of the antigen/antibody interaction and the abundance of the protein within the nucleus of a cell. The slight shifts for GFI1B and GFI1, both expressed from transgenes, is likely due to a relatively low affinity of the polyclonal antibodies available to detect them. In all stains performed, the overall shifts of both the IgG control and the experimental stain decrease upon isolation of nuclei, likely due to a loss of autofluorescence from cytoplasmic proteins. However, the stain for each transcription factor retains its shift above background in intact nuclei, while the shift for BCL2 disappears. Taken together, these data suggest that flow cytometry is a reliable method for determining nuclear localization of proteins.

We have developed and described companion methods for 1) detecting poorly expressed proteins in subsets of heterogeneous cell populations using low-affinity 


\section{Figure 5. Retention of nuclear stains upon removal of cytoplasm and cellular}

membrane. Microscopic analysis of Hoechst-stained GFP-positive thymocytes confirmed removal of cytoplasmic contents upon resuspension in NIM. The FACS image (cells) represents merged GFP/ Hoechst fluorescence while the NIM image is only the Hoechst fluorescence. No GFP fluorescence was observed in isolated nuclei. Histogram overlays indicate the expression of BCL2, EGR1, GFI1B, and GFI1, (left to right) in total thymocytes. The overlays include the specific IgG control for the experimental antibody (dashed line) and the experimental stain (bold line). Plots for intact cells are shown above plots for isolated nuclei. The nuclei are negative for BCL2, but positive for EGR1, GFI1B, and GFI1. Note: Microscopy performed by Michael K. Tanner, Institute for Cellular Therapeutics, University of Louisville. 
Figure 5
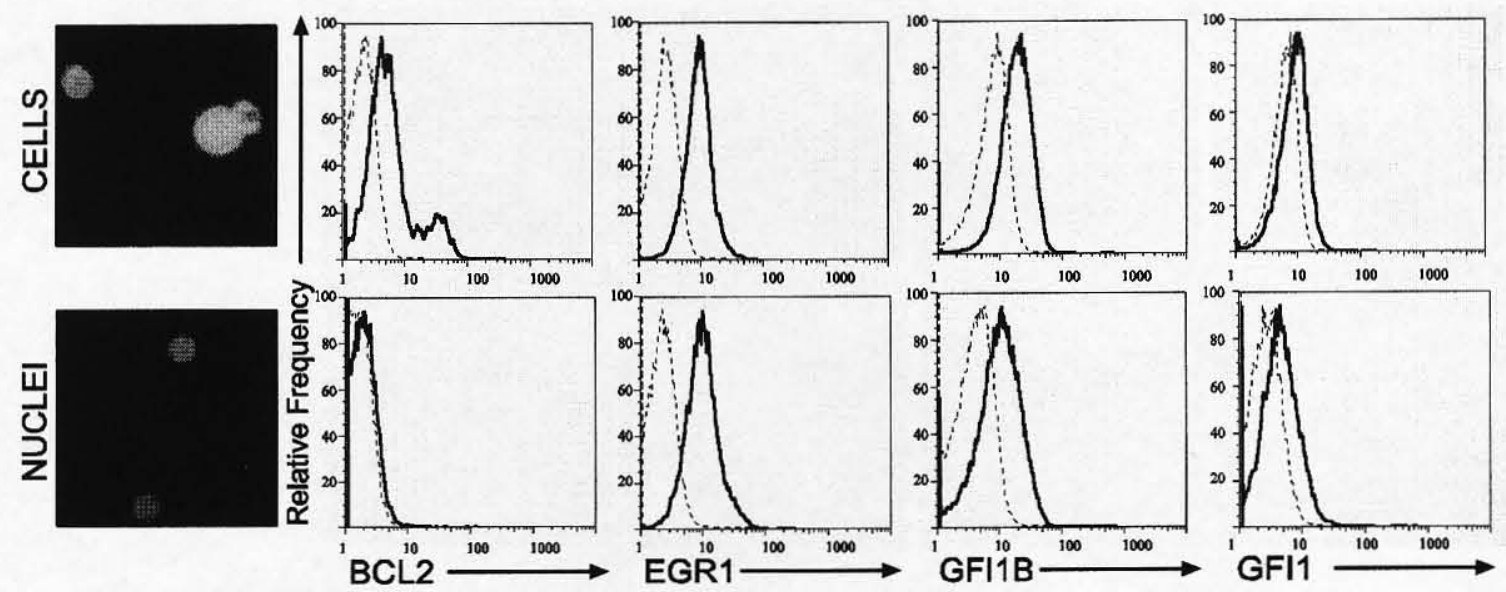
polyclonal antibodies and 2) confirming nuclear isolation of transcription factors. These methods combine to provide simple, reliable, and fast alternatives to traditional methods. 


\section{CHAPTER III}

\section{GFI1B EXPRESSION LEADS TO DEFECTS IN T-CELL ACTIVATION, IL7-Ra EXPRESSION AND T-CELL LINEAGE COMMITMENT}

\section{Introduction.}

$\mathrm{T}$ cell differentiation in the thymus is dependent upon signaling through the $\mathrm{T}$ Cell Receptor (TCR) and is characterized by the resulting changes in expression patterns of CD4 and CD8 surface coreceptor molecules. Early thymocyte precursors are CD4$\mathrm{CD}^{-}$(double negative, $\mathrm{DN}$ ) and are signaled to differentiate first into $\mathrm{CD} 4^{+} \mathrm{CD} 8^{+}$(double positive, DP) thymocytes. Further differentiation of DP thymocytes into mature $\mathrm{CD}^{+} 8^{-}$ single positive (CD4 SP) or $\mathrm{CD}^{-} 8^{+}$(CD8 SP) $\mathrm{T}$ cells is triggered by the engagement of TCRs on the immature $\mathrm{TCR}^{\text {hi }} \mathrm{CD} 4^{+} \mathrm{CD} 8^{+}$thymocytes by self-peptide/MHC complex on thymic epithelial cells $(28 ; 29)$. Negative selection eliminates immature DP thymocytes through clonal deletion of those $\mathrm{T}$ cells that have high affinity for self peptide and thus are potentially autoreactive (30). Positive selection occurs when low-affinity TCRligand interactions trigger a signal for survival and results in termination of one or the other CD4 or CD8 coreceptor molecule. The choice of which coreceptor to extinguish is referred to as lineage commitment.

The Growth Factor Independence-1 (GFI1) and GFI1B proteins are closely related nuclear oncoproteins that may regulate cytokine pathways. Gfil was originally identified as the gene upregulated by insertion of Moloney murine leukemia virus 
(MoMLV) in a thymic lymphoma that was selected for its ability to grow in the absence of the T-cell cytokine IL-2 (8). Forced expression of GFI1 in the IL-2-dependent parental cell line potentiates the outgrowth of IL-2 independent cell lines, without inducing IL-2 (8;9). GfilB was identified by low stringency hybridization screening with a cDNA probe encoding the zinc-finger region of Gfil (10). GFI1 and GFI1B are 97\% homologous in the carboxy-terminal 165 amino acids that code for six Cys-His zincfingers. An amino-terminal 20-amino-acid SNAG domain, responsible for nuclear localization and transcriptional repressor function, is also highly conserved (9). In contrast, the 236 intervening amino acids between the GFI1 SNAG and zinc-finger domains bear no homology to the corresponding 145 amino acids of GFI1B. Both proteins bind to virtually identical DNA consensus sequences and function as transcriptional repressors in a SNAG-dependent manner $(9 ; 10)$. GFI1 is mildly antiapoptotic and inhibits growth arrest of IL-2 dependent $\mathrm{T}$ cell lines under conditions of limiting IL-2 (9;31), while GFI1B inhibits both IL-6-induced differentiation and growth arrest of M1 myelomonocytic cells (10). Mice deleted for Gfil have altered inflammatory responses and differentiation in the myeloid lineage (17), while mouse embryos deleted for GfilB die in utero due to a lack of definitive erythropoiesis (18).

$G f i 1$ and $G f i 1 B$ are differentially expressed in lymphoid compartments. Northern analysis reveals that $G f i l$ is expressed in the bone marrow and thymus, with low-level expression in the spleen, whereas $G f i l B$ is expressed in the bone marrow and spleen, with low-level expression in the thymus (10). Both $G f i l$ and $G f i 1 B$ show regulated expression during $\mathrm{T}$ cell development, but $G f i 1 B$ expression is terminated in mature thymocytes. Gfil message is not expressed in $\mathrm{G}_{0}$ splenic $\mathrm{T}$ cells, but is induced upon $\mathrm{T}$ cell activation 
$(8 ; 20)$. Transgenic expression of GFI1 and GFI1B in T cells allowed us to determine the functional basis for differential expression of these factors. Transgenic expression of GFI1 potentiates T-cell activation. In contrast, ectopic expression of GFI1B in T cells results in defective T-cell activation, lower numbers of peripheral $\mathrm{T}$ cells, a reduction in IL-7R $\alpha$ expression and a developmental block to CD8 SP T cells. The block to CD8 SP development is mitigated by forced expression of BCL2 or GFI1. These data indicate that GFI1 and GFI1B are not redundant for $\mathrm{T}$ cell activation function, and implicate integration of activation and survival signaling in CD8 lineage commitment.

\section{Materials and Methods.}

Mice. The generation of the $c d 2-G f i l$ transgenic mice has been described (20). The $l c k$ Gfil transgenic mouse (line $3 \mathrm{~A}$ ) was generated by cloning the rat $G f i l$ cDNA into the BamHI site of the TLC vector (32). This vector contains a 3.2-kb fragment of the mouse $l c k$ proximal promoter and a $2.2-\mathrm{kb}$ fragment of the human growth hormone $(G H)$ gene, which provides exons and introns for splicing and polyadenylation sequences. A 2.2-kb fragment of the 3' locus control region of the human $C D 2$ gene is located downstream of the $G H$ to obtain copy-number- and insertion-site-independent levels of expression. The GFI1B transgenic mice (lines 5B and 5C) were constructed in an identical manner to the GFI1 transgenic except the construct contained the cDNA for mouse $G f i 1 B$ inserted into the BamHI site. The GFI1 and/or the GFI1B transgene ( 1 to $5 \mathrm{ng} / \mu \mathrm{l}$ ) was microinjected into C57BL/6J (Jackson Laboratory) eggs according to standard methodology in the Laboratory of Immunopathology, NIAID, NIH. 
$\mathrm{RAG}^{-1-} / \mathrm{HY}$ mice (Taconic), and E $\mu$-BCL2-25 transgenic mice (Jackson

Laboratory) (33) were purchased from commercial vendors. All mice were on a C57BL/6 J background and bred in the Baxter Barrier animal care facility at the University of Louisville School of Medicine and housed under specific pathogen-free conditions. GFI1 and GFI1B transgenic daughters who were heterozygous for the RAG mutation, as well as the HY TCR transgenes were then backcrossed with their RAG2 ${ }^{-/ \text {, }}$ $\mathrm{HY} \mathrm{TCR}^{+}$fathers. Colonies were expanded by intercrossing of littermates. BCL2/GFI1B and cd2-GFI1/GFI1B bi-transgenics were generated in a similar manner. All animal work performed was reviewed and approved by the University of Louisville Institutional Animal Care and Use Committee.

Antibodies. Antibodies with the following specificities were used for cell stimulations: $\mathrm{CD} 3 \varepsilon$ (145.2C11) and CD28 (37.51). Antibodies with the following specificities were used for staining of thymocytes and splenocytes: CD4 (RM4-5 and GK1.5), IL-7R $\alpha$ (A7R34; eBiosciences), CD8 $\alpha$ (53-6.7), CD3e-( 145-2C11), TCR- $\alpha \beta$ (H57-597), TCR V $\beta 8$ (F32), CD24 $\alpha-(\mathrm{M} 1 / 69), \mathrm{CD} 69$ (H1.2F3), and CD25 (PC61). Antibodies were purchased from BD-Pharmingen unless otherwise noted. Intranuclear staining was performed using anti-GIF1B goat polyclonal IgG (sc-8559), anti-GFI1 goat polyclonal IgG (sc-8558), normal goat IgG (sc-2028) control, and secondary bovine anti-goat IgGFITC (sc-2348), all from Santa Cruz Biotechnology.

Preparation of Cell Suspensions. Thymi from 4- to 6- week-old mice were removed and disrupted between frosted ends of glass slides and washed twice with Medium 199 (Gibco BRL Life Technologies, Gaithersburg MD). Cells were obtained from spleens by perfusion with $10 \mathrm{ml}$ Medium 199. Both thymocyte and splenocyte cell debris was 
depleted by passage through Nytex nylon mesh screens. Splenocytes were treated with ammonium chloride-potassium bicarbonate (ACK) solution (150mM NH4Cl and $10 \mathrm{mM}$ KHCO3) to lyse red blood cells. For experiments requiring isolation of T cells, splenic cell preps were depleted of other cell types by the use of $\mathrm{T}$ cell enrichment columns (R\&D Systems). All cells were counted with a Coulter Counter model Z2 (Coulter, Miami FL) and viability assayed by trypan blue exclusion.

Flow Cytometric Analysis. Cell surface staining was performed by incubating $1 \times 10^{6}$ cells with mAbs at varying concentrations in FACS $\otimes$ media (HBSS with $0.1 \%$ BSA, $0.1 \%$ Sodium Azide, and $0.036 \%$ Sodium Bicarbonate) for $20 \mathrm{~min}$ on ice. Stained cells were washed twice with FACS $₫$ media and fixed in $1 \%$ formaldehyde (Polysciences, Warrington, PA). For intracellular staining, cells were fixed in $2 \%$ formaldehyde in PBS and permeabilized and stained in PBS $+5 \%$ fetal bovine serum and $0.5 \%$ Triton $\mathrm{X}-100$. Flow cytometry was performed on a FACSCaliburTM, FACSVantageSE, or FACStar flow cytometer using standard CELLQuestTM acquisition. Data were analyzed using CELLQuestTM (Becton Dickinson) and FlowJoTM (TreeStar) software. The absolute cell numbers of gated cells per thymus or spleen were calculated by multiplying the percentage of each population with the total number of cells per thymus or spleen respectively.

Northern, Western and RT-PCR Analyses. RT-PCR analyses were performed as previously published $(34 ; 35)$. The sequences of the Gfil-specific primers were 5 'CACACCTTCATCCACACAGG-3', 5'-GATGAGCTTTGCACACTGGA-3', and the probe was 5'-TACCGTGAGGATGTCTTCCC-3'. The sequences of the GfilB-specific primers were 5'-AGCACAGAGTCTCCCTTGGA-3', 5'- 
CAAAGGTTTTGCCACAGACA-3', and the probe was 5'-

ACCCCTCATGGGCTAGAAGT-3'. The GfilB pattern was confirmed with the primers 5'-GAGCAGCATACTCACGTCCA-3', 5'-TTCATGTCCGACTTCTGGTG-3', and the probe was 5'-CAAAGCCTTCAAGCGTTCAT-3'.

Western blotting with antibodies against GFI1 (sc-6357), GFI1B (sc-8559, Santa Cruz)), GFI1 and GFI1B (sc-6357), p27 (BD Pharmingen 554069) and IRF1 antibody (sc-640) was performed as follows. Single cell suspensions of primary thymocytes were lysed at a concentration of $10-20 \times 10^{6}$ cells $/ 100 \mu$ l SDS lysis buffer ( $25 \mathrm{mM}$ Tris $\mathrm{pH} 7.5$, $10 \%$ glycerol, and 2\% SDS supplemented with Protease Inhibitors Complete (Roche) and 2mM PMSF. Protein concentration was determined using the BCA Protein Assay Reagent (Pierce, Rockford IL), and $75 \mu \mathrm{g}$ of cellular extract was run on a $10 \%$ SDSpolyacrylamide gel, transferred to Immobilon-PVDF (Millipore, Bedford, MA) and blocked for 1 hour at room temperature (RT) in blocking buffer (5\% milk, $20 \mathrm{mM}$ Tris, $\mathrm{pH}$ 7.3, 6.85 mM NaCl, 0.1\% (v/v) Tween-20, $0.5 \mathrm{~g} / \mathrm{L} \mathrm{MgCl}$ ). Membranes were incubated overnight at $4{ }^{\circ} \mathrm{C}$ in primary antibody diluted in 5\% protease-free BSA (Fisher Biotech, Pittsburgh, PA), then HRP-conjugated secondary antibody (Amersham) for one hour at RT. Blots were developed using ECL reagents (Amersham,). For Western analysis of sorted thymocytes and purified T cells, $1 \times 10^{6}$ cells were resuspended in $15 \mu \mathrm{l}$ of lysis buffer (50 mM HEPES pH 7.8, $450 \mathrm{mM} \mathrm{NaCl}, 0.2$ mM EDTA, 25\% glycerol, $1 \%$ NP40 (36), Protease Inhibitors Complete (Roche), and 2 mM PMSF), then sonicated using a Misonix Sonic Dismembrator with microprobe tip. $4 \mathrm{X}$ loading buffer was added, and the lysates were boiled. The entire contents of the lysate were loaded onto a 
denaturing SDS polyacrylamide gel, and Western blotting was performed as described above.

Cell Stimulation and Proliferation. Single cell suspensions of spleen cells in RPMI 1640 (Gibco BRL Life Technologies, Gaithersburg, MD) supplemented with 5\% fetal bovine serum, L-glutamine, penicillin, streptomycin, gentamycin, 2\% HEPES (all from Gibco BRL Life Technologies) and 0.1\% 2-mercaptoethanol (Sigma) were plated in 96well round-bottom plates (Corning, Corning, NY) at a density of $1 \times 10^{5}$ per well in 100 $\mu$ l. Stimuli were added as indicated at a range of concentrations to assess dose dependency. The stimuli were low-endotoxin, no-azide anti-CD3 (145.2C11), anti-CD28 (37.51) (both antibodies from Pharmingen, San Diego, CA), and recombinant human IL2 (Chiron, Emeryville, CA). Cells were cultured for 48 or 72 hours, then pulsed with $\left[{ }^{3} \mathrm{H}\right]$ thymidine $(1 \mu \mathrm{Ci})$ (Amersham, Piscataway, NJ), and harvested 18 hours later using a TOMTEC-Harvester96/ Model Mach II (Wallac, Akron, OH). Proliferation was determined by measuring radioactivity (Wallac 1205-SP2 Betaplate Counter). Coreceptor Reversal. Purified DP thymocytes ( $>96 \%$ ) were obtained by panning with IgM anti-CD8 (83-12-5) coated plates. DP thymocytes $\left(5 \times 10^{6} / \mathrm{ml}\right)$ were first placed into "signaling cultures" and stimulated for 12-18 hours with a combination of phorbal-12myristate-13-acetate $(0.6 \mathrm{ng} / \mathrm{ml})$ and ionomycin $(0.6 \mu \mathrm{g} / \mathrm{ml})(\mathrm{P}+\mathrm{I}$; Calbiochem $)(37)$. At the conclusion of signaling culture, cells were harvested, washed, and placed into nonstimulatory "recovery cultures" for an additional $12-16 \mathrm{hr}$. Cells were stained for $\mathrm{CD} 4$ and $\mathrm{CD} 8$ expression and $\mathrm{CD} 4^{+} \mathrm{CD} 8{ }^{-}$cells were obtained by electronic sorting of the stained cells. The purified $\mathrm{CD}^{+} 8^{-}$cells were further cultured in "postrecovery cultures" in the presence or absence of $6 \mathrm{ng} / \mathrm{ml}$ recombinant mouse IL-7 (Calbiochem, San Diego, 
CA) overnight, after which they were harvested and stained for CD4 and CD8 expression. All cultures were performed at $37^{\circ} \mathrm{C}$ in $5 \% \mathrm{CO}_{2}$ humidified air atmosphere in RPMI 1640 supplemented with $5 \times 10^{-5} \mathrm{M} 2$-mercaptoethanol and $10 \%$ FCS that had been depleted of endogenous steroids by pretreatment with $0.5 \%$ Norit A charcoal and $0.05 \%$ dextran for $30 \mathrm{~min}$ at $56^{\circ} \mathrm{C}$.

\section{Results.}

\section{Gfil and $G$ filB are regulated during $\mathrm{T}$ lymphopoiesis.}

We examined the steady-state mRNA levels of $G f i 1$ and $G f i 1 B$ during T lymphopoiesis (Figure 6A). Thymocyte populations were sorted, RNA was extracted, and reverse transcriptase-polymerase chain reaction (RT-PCR) was performed to detect ribosomal S16 expression (34;35). The products of the reaction were analyzed by Southern blot with a radiolabelled $S 16$-specific oligonucleotide probe. The signal was quantified by phosphorimager, and the samples were normalized to obtain equivalent $S 16$ signal from each template. Subsequent analysis of $G f i 1$ expression in the S16-normalized cDNA templates revealed low-level signal in $\mathrm{CD}^{-\mathrm{CD}} 8^{-} \mathrm{CD}^{-} 4^{+} \mathrm{CD} 25^{-}$cells (DN1) and 10 fold greater levels in $\mathrm{CD}^{-} \mathrm{CD}^{-} \mathrm{CD} 44^{+} \mathrm{CD} 25^{+}$(DN2) thymocytes. The transition between DN1 and DN2 corresponds to T lymphocyte lineage commitment. Signal intensity from the GfiI RT-PCR product gradually increases to double the DN2 levels at the $\mathrm{CD}^{+} \mathrm{CD}^{+} \mathrm{TCR}^{\mathrm{hi}}$ stage, which contains cells that have been recently positively selected and are about to undergo lineage commitment. Gfil RT-PCR product levels then decrease 10 fold in CD4 and CD8 single positive thymocytes. In striking contrast, the signal levels of probed and quantified GfilB RT-PCR product were low, but increased at 
Figure 6. Expression of endogenous GFI1 and GFI1B. A. Blotted and probed RTPCR analyses of $G f i 1$ and $G f i l B$ in reverse-transcribed thymocyte-subset cDNA templates. cDNA templates were normalized for equivalent signal from blotted, probed S16. Primer/probe pairs for $G f i l$ and $G f i l B$ were applied to the normalized cDNA templates to determine the relative Gfil and GfilB RT-PCR product levels in thymocyte subsets. Note: These data were generated by Dr. Sabine Herblot and Dr. Trang Hoang (Research Institute of Montreal, Montreal, Quebec) using PCR primers and conditions designed and optimized by Loretta Doan. B. Western blot analysis of sorted thymocyte subsets. $1 \times 10^{6}$ sorted cells of each subpopulation were lysed, and the total lysate was probed with anti-GFI1 (top panel) or anti-p27 (bottom panel) as a loading control. GFI1 protein levels are moderate in DP thymocytes and intermediate $\mathrm{CD} 4^{+} \mathrm{CD} 8^{\text {lo }}$ cells, but increase in mature CD4 and CD8 SP cells. Note: These data were generated jointly by Loretta Doan and Dr. Mary Kate Kitay. C. Flow cytometric analysis of GFI1 expression in thymocyte subsets. Intranuclear staining of surface stained fixed thymocytes yielded an expression profile for GFI1 similar to that observed in C. Specifically, the level of GFI1 protein increases in SP thymocytes. The pattern of GFI1 expression in DN thymocytes has been published (36). D. Flow cytometric analysis of GFI1B expression in thymocyte subsets. Intranuclear staining of surface stained fixed thymocytes revealed expression of GFI1B in the relatively rare $\mathrm{CD} 4{ }^{-} \mathrm{CD} 88^{-} \mathrm{CD} 44^{-}$populations of cells. The average change in mean fluorescence intensity is indicated below each graph, and is

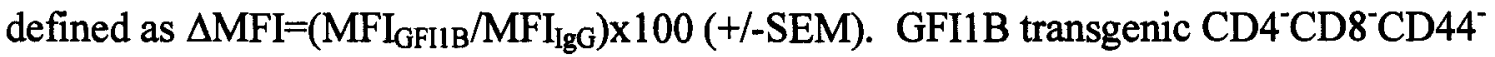
thymocytes have a higher MFI in comparison to WT cells; whereas RAG2 $2^{-/}$thymocytes, which lack TCR signaling (38), do not express detectable levels of GFI1B. 
Figure 6

A.

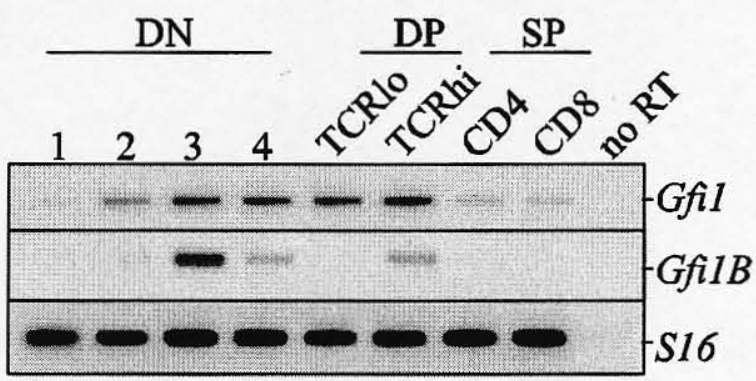

B.

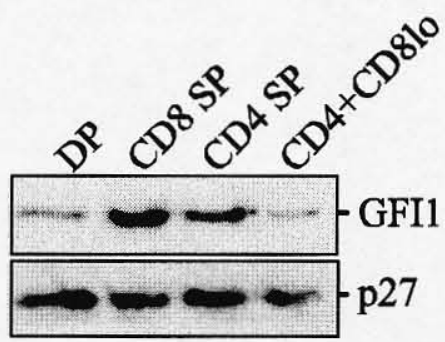

C.

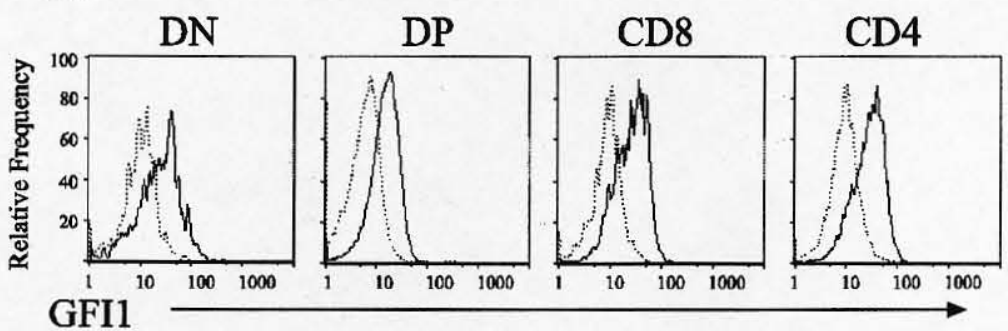

D.
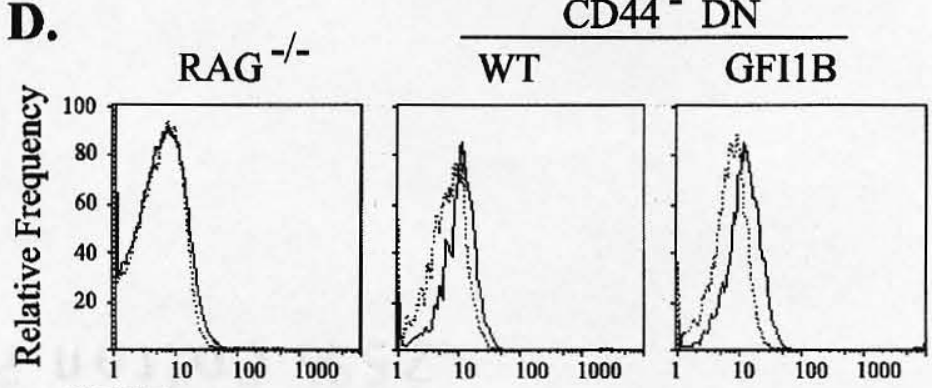
GFI1B

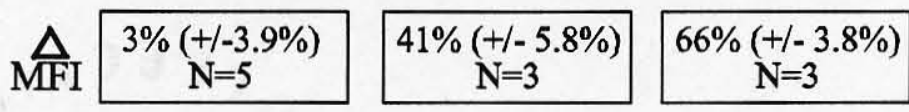


stages corresponding to $\mathrm{T}$ cell receptor beta-chain selection (DN3), and positive selection (DP TCR ${ }^{\mathrm{hi}}$ ). In the thymus, Gfil expression is gradually induced upon induction of the T cell differentiation program, whereas low-level GfilB expression correlates with positive selection events.

\section{GFI1 and GFI1 B are expressed in normal thymocytes.}

To examine the level of GFI1 in thymocyte subsets we performed Western analysis on one million sorted DP, CD8 SP, CD4 SP and CD4 $4^{+} \mathrm{CD} 8^{\text {lo }}$ thymocytes (Figure 6B). The level of GFI1 does not differ between the bulk of DP thymocytes and those poised to make a lineage commitment step $\left(\mathrm{CD} 4^{+} \mathrm{CD} 8^{\text {lo }}\right)$; however, the level of GFI1 is dramatically higher in SP thymocytes. To confirm these data, we examined thymocyte expression of GFI1 by intranuclear staining and flow cytometry. As seen in the Western blot data, flow cytometric analyses revealed higher levels of GFI1 protein in SP thymocytes (Figure 6C). The upregulation of Gfil message in DP thymocytes, with subsequent increase in GFI1 protein in SP cells, suggests that GFI1 may play a role in the transition between these two developmental stages.

RT-PCR analyses revealed restricted expression of GfilB in relatively rare thymocyte subsets (Figure 6A). Not surprisingly, Western analysis and intranuclear stains for GFI1B failed to reveal GFI1B expression in bulk thymocytes (data not shown). Therefore, we focused on a flow-cytometric analysis of the relatively rare $\mathrm{CD}^{-} 4^{-} \mathrm{DN} 3$ and DN4 thymocytes that appear to express the highest levels of GfilB message (Figure 6A). We first examined the DN3 and DN4 cells from GFI1B-transgenic mice (detailed below). A comparative $66 \%$ shift in mean fluorescence intensity $(\Delta \mathrm{MFI}=$ $\mathrm{MFI}_{\mathrm{GFIIB}} / \mathrm{MFI}_{\mathrm{IgG}} \times 100$ ) between the control IgG antisera and GFI1B-reactive antisera 
indicates that GFI1B protein is present (Figure 6D). In a similar manner, analysis of the DN3 and DN4 cells from non-transgenic littermates revealed a 41\% shift in MFI between control and GFI1B-specific antisera stains (Figure 6D).

The RT-PCR data (Figure 6A) indicate that GfilB expression correlates with positive selection events. To explore this correlation we examined thymocytes from $\mathrm{RAG}^{-/}$mice, which are arrested at the DN3 stage because they lack the pre-TCR selection signal that follows RAG-mediated rearrangement of the TCR-beta chain. Flowcytometric analyses reveal no difference in MFI between control and GFI1B-reactive antisera in RAG2 ${ }^{-/-}$thymocytes (Figure 6D). These data indicate that signals from the pre-TCR may be required for GFI1B expression.

\section{Transgenic Expression of GFI1 and GFI1B.}

GFI1 and GFI1B bind the same DNA sequence and repress transcription in a manner dependent on the SNAG repressor domain $(9 ; 10)$, suggesting the possibility that these factors are redundant. High-level transgenic expression of GFI1 in the thymus results in a block to $\mathrm{T}$ cell development at a stage corresponding to selection of cells after successful formation of the TCR $\beta$ chain (36). Given the thymic phenotype of GFI1 overexpression, we constructed transgenic mice expressing GFI1 or GFI1B in developing and mature T cells (Figure 7A). Transgene-specific Northern analysis revealed that GFI1-transgenic founders had moderate expression while GFI1B-transgenic founders had higher levels of expression; representative lines are shown (Figure 7B). Western analysis of total thymocytes and of column-enriched splenic $\mathrm{T}$ cells indicates that the transgenic GFI1B protein is expressed in both the thymus and the periphery (Figure 7C). Finally, flow cytometric analysis of the GFI1B-transgenic thymocyte populations revealed 
Figure 7. Expression of transgenic GFI1 and GFIB. A. Schematic representation of the GFI1 - and GFI1B-transgene constructs. The rat cDNA of Gfil (8) and mouse cDNA of $G f i 1 B(10)$ was placed under the control of the $l c k$ proximal promoter and $2.2 \mathrm{~kb}$ human CD2 enhancer, with human growth hormone (GH) gene (32). B. Northern blot analysis of total thymus or spleen RNA from representative GFI1 (line 3A) and GFI1B (line 5B) transgenic lines hybridized with transgene-specific probes. Total RNA from transgenic animals revealed moderate levels of transgenic Gfil in both thymus and spleen, while transgenic GfilB is expressed at higher levels. C. Western blot analysis of transgenic GFI1B in thymic or column-purified splenic T-cell whole-cell protein extracts. Cell extracts from 2 control (WT) and 2 GFI1B-transgenic mice were analyzed by Western blot with an antibody specific for the last 20 amino acids of GFI1 (cross reactive to GFI1B). Densitometric analysis revealed that transgenic GFI1B is expressed at fivefold higher levels than is endogenous GFI1 in non-transgenic littermate thymocytes. Lysates probed with antiserum against IRF-1 act as a control for loading. D. Flow cytometric analysis of GFI1B expression in GFI1B-transgenic thymocyte subsets. Transgenic expression of GFI1B is observed in all thymocyte subsets, though the level of expression is somewhat lower in CD8 SP cells. 
Figure 7

A.

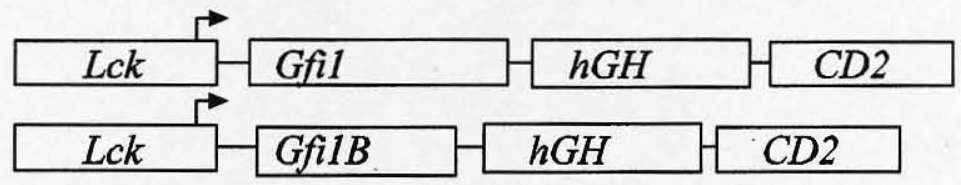

B. Transgenic: GFI1 GFI1B

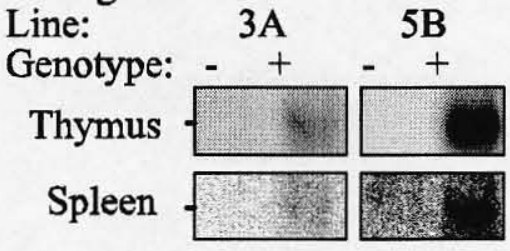

c.

Thymus
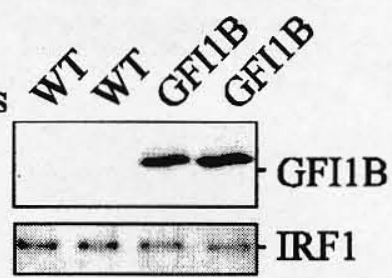

Splenic T cells

D.

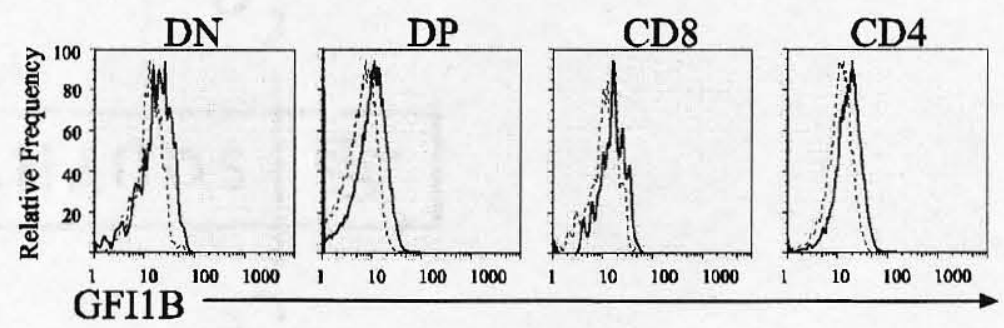


transgenic GFI1B expression in DN, DP, CD8 SP and CD4 SP thymocytes (Figure 7D).

To explore the subcellular localization of transgenic GFI1 and GFI1B, the cytoplasm of permeabilized and stained thymocytes was removed using NP40. Stripping the cytoplasm resulted in a loss of signal for the mitochondria-localized BCL2, but did not alter the $\triangle$ MFI of thymocytes stained for GFI1 or GFI1B (data not shown), indicating that the targets of the respective antisera are nuclear. Given that these antisera are specific for GFI1 and GFI1B in Western analysis, our flow cytometric data indicate that transgenic GFI1 and GFI1B are localized to the nucleus of T cells.

\section{Transgenic expression of GFI1 enhances T cell response to CD3 crosslinking and}

\section{IL-2.}

GFIl was previously shown to confer IL-2 independence to rat T-cell lymphomas (8;31). IL-2 is a critical T-cell cytokine during activation, and though peripheral lymphocytes do not express detectable levels of Gfil, activation signals induce Gfil within 30 minutes (20). Moreover, transgenic expression of GFI1 was previously shown to mildly increase $\left[{ }^{3} \mathrm{H}\right]$-thymidine uptake given a fixed amount of $\mathrm{CD} 3$ crosslinking antibody (20). To further examine the effect of GFI1 on T-cell activation potential, spleen cells from 4-6 week-old mice were stimulated by titration of a CD3-crosslinking antibody. T cells from mice expressing GFI1 from either the $L c k$-promoter driven transgene (Figure 7A), or a previously published $C D 2$-promoter driven transgene (20), proliferated at a higher rate than $\mathrm{T}$ cells from control mice as evidenced by enhanced $\left[{ }^{3} \mathrm{H}\right]$-thymidine uptake (Figure 8A) and by increased numbers of cells in each cellular division as evidenced by CSFE staining (data not shown). 
Figure 8. GFI1 potentiates, whereas GFI1B inhibits, T-cell activation. A and B. Spleen cells from 4-6 week old GFI1 transgenic mice (line 3A or a CD2 promoter-driven GFI1 transgenic (20)) and nontransgenic littermate control mice were isolated and stimulated with either a dose titration of plate-bound antibody to $\mathrm{CD} 3 \varepsilon$ (A) or a low level of anti-CD3ع $(0.06 \mu \mathrm{g} / \mathrm{ml})$ and increasing amounts of human rIL-2 (B) and cultured for 48 hours. $\left[{ }^{3} \mathrm{H}\right]$ thymidine $(1 \mu \mathrm{Ci})$ was added and plates were incubated for 18 hours. Proliferation was measured as CPM of $\left[{ }^{3} \mathrm{H}\right]$ thymidine incorporation. Error bars indicate standard error of the mean. Note: These data were generated by Dr. Michael Alexander. The results shown in Panel A were reproduced by Loretta Doan. C. Spleen cells from 46-week-old GFI1 transgenic mice, as well as littermate controls, were stained with antibodies to CD4 and CD8 then analyzed by flow cytometry. A representative FACS profile is shown. No difference was observed in total number of splenocytes or in splenic T cell numbers between GFI1 transgenics and littermate controls of either line. Note: These data were generated by Dr. Mary Kate Kitay and reproduced by Loretta Doan. D. Spleen cells from 4-6 week old GFI1B transgenic mice (lines 5B and 5C) were activated as in (A) for either 48 hours (left panel) or 72 hours (right panel) before the addition of $\left[{ }^{3} \mathrm{H}\right]$ thymidine. E. Spleen cells isolated from 4-6-week-old GFI1B transgenic line 5B, as well as littermate controls, were stained with antibodies to CD8 and CD4 and analyzed by flow cytometry. A representative FACS profile is shown. Absolute spleen cell numbers from all mice examined were determined and are expressed in the table below as cell numbers $\times 10^{6}{ }^{ \pm}$SEM in the table below. Note: These data were generated by Dr. Mary Kate Kitay and reproduced by Loretta Doan. 
Figure 8

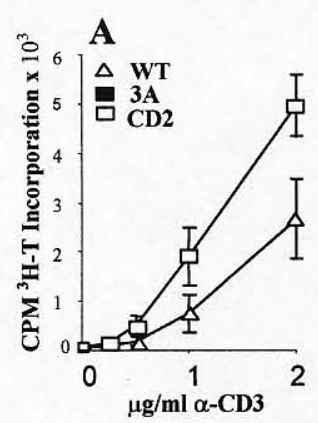

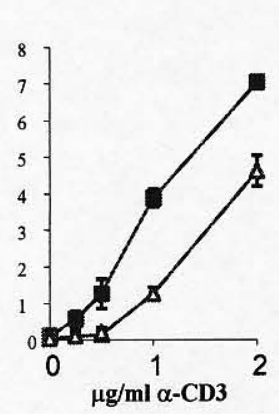

B

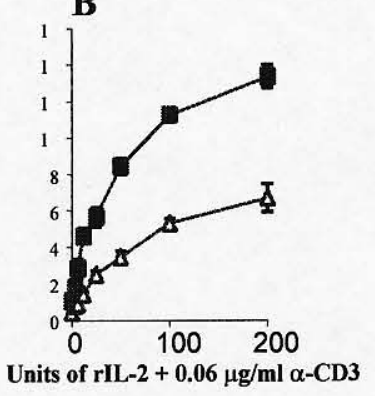

D

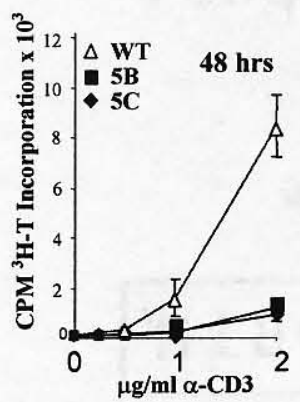

C
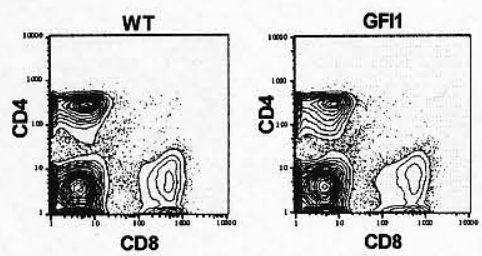

E
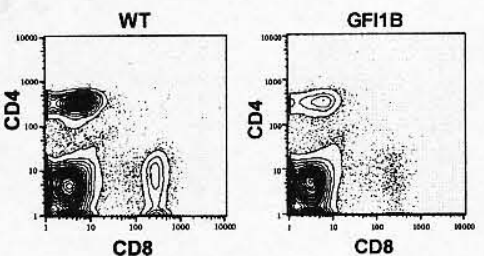

\begin{tabular}{c|c|c}
$\begin{array}{c}\text { Peripheral } \\
\text { subset }\end{array}$ & WT $(\mathbf{n}=4)$ & $\begin{array}{c}\text { GFW B } \\
(\mathbf{n}=4)\end{array}$ \\
\hline CD4+ & $8.69 \pm 2.4$ & $2.85 \pm 0.74$ \\
\hline CD8+ & $1.50 \pm$ & $0.69 \pm 0.21$ \\
& 0.64 & $70.71 \pm$ \\
Total & $86.57 \pm$ & 11.02
\end{tabular}


To dissect the response of GFI1-transgenic splenic T cells to stimulation, we limited the amount of CD3 antibody, and added increasing amounts of IL-2. Again, we found that cells from GFI1-transgenic mice proliferated more vigorously in response to stimulation than cells from non-transgenic littermates (Figure 8B). A flow cytometric analysis of splenocytes from control and GFI1 transgenic mice show equivalent absolute cell numbers of total splenocytes and T cell subsets as delineated by the markers CD4, CD8, TCR beta, and CD3e (Figure 8C), or CD62L and CD44 (data not shown). Therefore, GFI1 potentiates the response to CD3 and IL-2 stimulation.

GFI1B transgenic mice display peripheral T lymphopenia and a profound defect in activation after CD3 cross-linking.

We next looked at the response of GFI1B transgenic splenic T cells to stimulation with anti-CD3E and found that they neither died (data not shown), nor proliferated substantially. Spleen cells from 4-6 week-old mice from two GFIlB-transgenic lines (5B or 5C, Figure 8D) were stimulated by addition of increasing quantities of a CD3crosslinking antibody. T cells from GFI1B transgenic mice proliferated at a substantially lower rate than $\mathrm{T}$ cells from control mice as evidenced by $\left[{ }^{3} \mathrm{H}\right]$-thymidine uptake, even when the activated cells were given an additional 24 hours incubation (Figure 8D, 48 hours versus 72 hours).

An unanticipated potential explanation for this observation came from the finding that spleen cells from GFI1B transgenic mice show significant reduction in the numbers of CD4 and CD8 T cells that could respond to CD3 stimulation (Figure 8E). Mature CD4 cells were reduced to $32 \%$ of wild type levels, while mature CD8 cells were reduced to $46 \%$ of wild type levels (Figure 8E). However, neither the TCR-expression level on 
splenic T cells (data not shown) nor the total number of splenocytes (Figure 8E) was significantly reduced in GFI1B transgenic mice. Since both the GFI1B-transgenic lines gave equivalent data, we focused on the 5B line for further studies.

The unresponsiveness of GFI1B lymphocyte populations to TCR-mediated activation signals could be due either to Tlymphopenia or to a defect in signaling. To determine the mechanism, $\mathrm{T}$ cells were purified by negative selection, normalized to

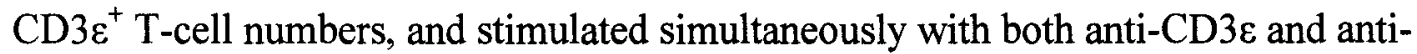
CD28 in a co-receptor activation assay. Co-stimulated GFI1B-transgenic cells showed a marked inability to proliferate as compared to cells from non-transgenic littermates (Figure 9A). In addition, GFI1B-transgenics demonstrated decreased proportions of cells expressing activation markers CD69 and CD25 (IL-2 receptor $\alpha$-chain) as compared to wild type cells (Figure 9B), as well as a decrease in fluorescence intensity of these markers on positive cells (data not shown). Therefore, GFI1B transgenic T cells are profoundly impaired in response to activation signals because of an intrinsic signaling defect, and not because of the overall reduction in $\mathrm{CD} 3 \varepsilon^{+} \mathrm{T}$ cell numbers. These data are diametrically opposed to our findings for GFI1-transgenic mice, which showed enhanced response to activation.

\section{Transgenic GFI1B expression alters thymocyte lineage commitment and maturation.}

In order to determine the cause of peripheral $\mathrm{T}$ lymphopenia, we examined the thymus. Thymocytes were stained with antibodies against CD4, CD8, and TCR $\beta$ and analyzed by flow cytometry. In GFI1B transgenic mice, the number of CD4 SP cells was considerably enhanced while there was a severe reduction in the development of CD8 SP 
Figure 9. The GFI1B-induced inhibition of activation is cell autonomous and includes defective upregulation of early activation markers. A. 4 week old GFI1B transgenic and control splenic $\mathrm{T}$ lymphocytes were purified by negative selection, normalized for numbers of $\mathrm{CD}^{+} \mathrm{T}$ cells, and then co-stimulated with antibodies to $\mathrm{CD} 3 \varepsilon$ and CD28. Proliferation was measured by $\left[{ }^{3} \mathrm{H}\right]$ thymidine incorporation and the stimulation index (fold induction over non-stimulated controls) was calculated. A representative of 3 experiments is shown. Note: These data were generated by Dr. Michael Alexander. B. Splenocytes from GFI1B transgenic (open symbols, black background) and littermate controls (closed symbols, white background) were stimulated with antibodies to $\mathrm{CD} 3$ for 24 hours then stained with antibodies to CD4, CD8, CD25 (circle) and CD69 (diamond) and analyzed by flow cytometry. $\mathrm{CD}^{+}$or $\mathrm{CD}^{+}$events were gated, and the percentages of $\mathrm{CD} 25^{+}$or $\mathrm{CD} 69^{+}$cells are depicted. Fewer GFI1Btransgenic splenocytes exhibit induction of either CD25 or CD69. Bars $=$ mean. Note: These data were generated by Amy Barber Schonck and reproduced by Loretta Doan. 
Figure 9
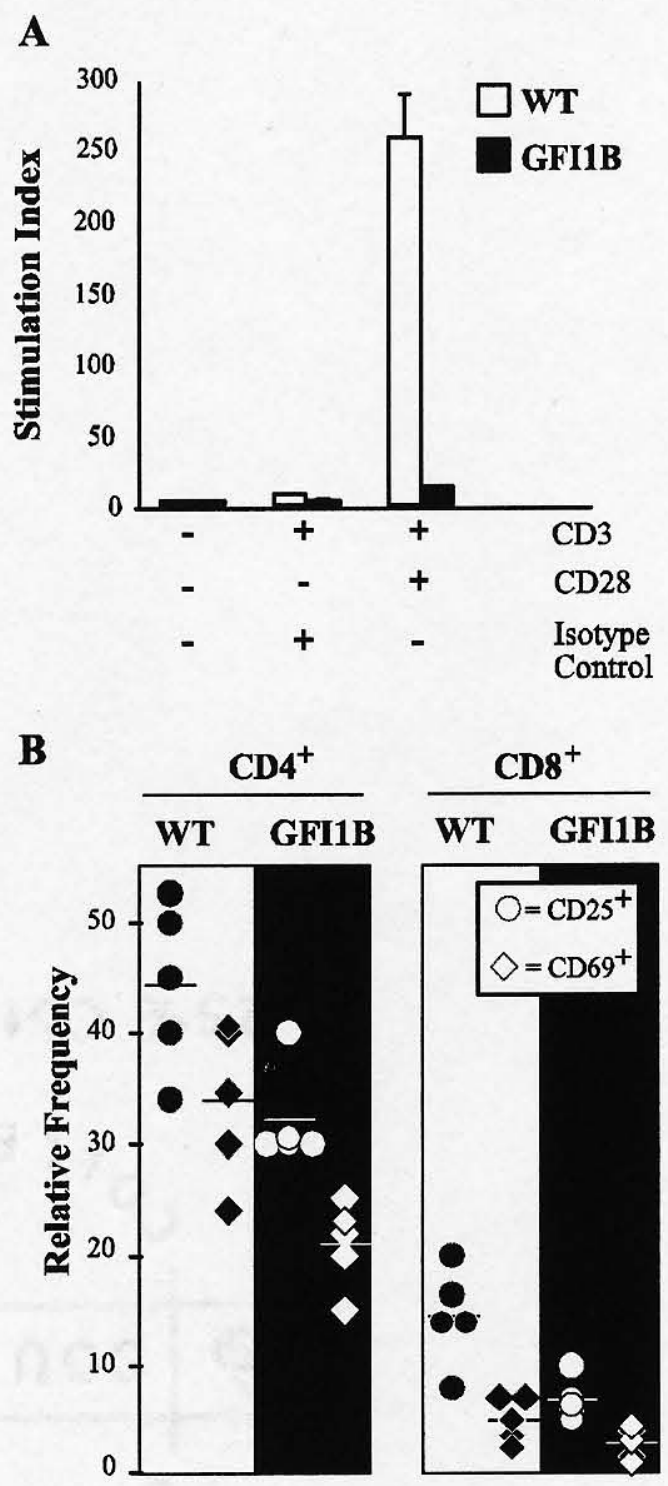
Figure 10. Transgenic expression of GFI1B in the thymus results in enhanced numbers of mature $\mathrm{CD4}^{+}$thymocytes and decreased $\mathrm{CD8}^{+}$populations. $\mathrm{A}$ and $\mathrm{B}$. Thymocytes from GFI1B transgenic and control littermates were stained with antibodies to TCR $\beta, \mathrm{CD} 4$, and $\mathrm{CD} 8$, and analyzed by flow cytometry. The average number of total thymocytes $\times 10^{6 \pm} \mathrm{SEM}$ is expressed above the plots, with percentage of cells in each gate in (A). In (B) cells are gated on TCR ${ }^{\text {int-hi }}$ events, and the CD4 versus CD8 FACS profiles are depicted. Thymocyte subsets are expressed as absolute cell numbers $\times 10^{6}$ per thymus ${ }^{ \pm}$SEM. Note: These data were generated by Dr. Mary Kate Kitay and reproduced by Loretta Doan. C. Thymocytes from 4-week-old GFI1B transgenic and control littermates were stained with antibodies to CD24 , CD4, and CD8 and analyzed by flow cytometry. FACS profiles depicted are gated on $\mathrm{CD}^{+}$events. Absolute thymocyte cell numbers from all mice examined were determined and are expressed as cell numbers $\times 10^{6 \pm}$ SEM. Note: These data were produced jointly by Loretta Doan and Dr. Mary Kate Kitay. 
Figure 10
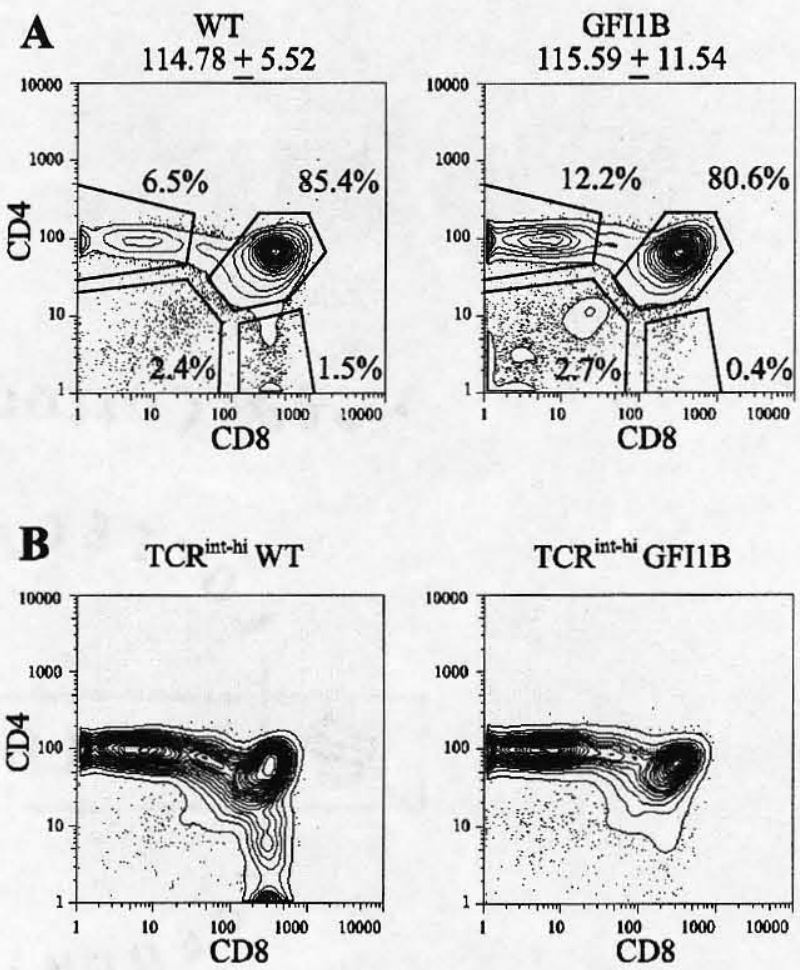

\begin{tabular}{|lcr|}
\hline TCR $^{\text {int-hi }}$ Subset & $\begin{array}{c}\text { WT } \\
(\mathrm{n}=4)\end{array}$ & \multicolumn{1}{c|}{$\begin{array}{c}\text { GFI1B } \\
(\mathrm{n}=4)\end{array}$} \\
\hline DN & $0.23 \pm 0.11$ & $0.22 \pm 0.04$ \\
DP & $10.77 \pm 1.38$ & $10.01 \pm 0.52$ \\
CD4 SP & $6.42 \pm 0.49$ & $9.66 \pm 0.66$ \\
CD8 SP & $0.93 \pm 0.03$ & $0.22 \pm 0.04$ \\
\hline
\end{tabular}
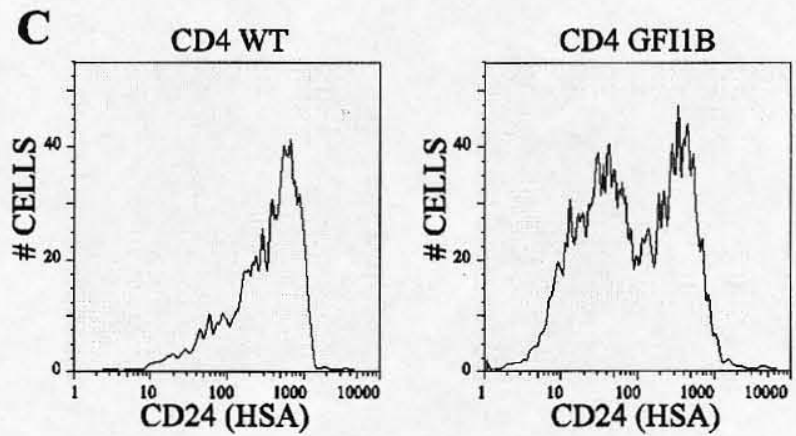

\begin{tabular}{|c|c|c|}
\hline $\mathrm{CD}^{+}$Subset & $\begin{array}{l}\text { WT } \\
(n=4)\end{array}$ & $\begin{array}{l}\text { GFI1B } \\
(n=6)\end{array}$ \\
\hline $\begin{array}{l}\text { CD24 }{ }^{\text {lo- }} \\
\text { CD24 }\end{array}$ & $\begin{array}{l}1.14 \pm 0.22 \\
4.83 \pm 0.07\end{array}$ & $\begin{array}{l}5.59 \pm 0.33 \\
5.56 \pm 0.64\end{array}$ \\
\hline
\end{tabular}


T cells (Figure 10A). The CD8 SP compartment contains both mature and immature intermediate single positive cells (CD8 ISP). CD8 ISP cells, in contrast to CD8 SP cells, do not have high level TCR expression. Therefore, the analysis was repeated through a $\mathrm{TCR}^{\text {int-hi }}$ gate, which would include CD4 SP, CD8 SP thymocytes and their immediate precursors (39). The ratio of $\mathrm{CD}^{+}$to $\mathrm{CD}^{+}$cells was increased in the $\mathrm{TCR}^{\text {int-hi }}$ population from a normal ratio of 7:1 to a ratio of 32:1 in the GFI1B-transgenic mice (Figure 10B). Therefore, few mature CD8 SP cells are generated in the GFI1B transgenic mice. Moreover, the lower numbers of CD8 SP cells provides a potential explanation for peripheral CD8 lymphopenia.

We next examined the CD4 SP population. As CD4 SP thymocytes mature they downregualte CD24/HSA and exit the thymus. Therefore, the most mature CD4 SP thymocytes express low levels of CD24 and are relatively rare in wild type mice, while less mature CD4 SP thymocytes express higher levels of this marker (39-41). We found that the number of immature CD4 SP thymocytes $\left(\mathrm{CD} 24^{\mathrm{hi}}\right)$ was relatively normal in GFI1B-transgenic mice; however, the number of mature CD4 SP thymocytes was increased five fold (Figure 10C). These data are supported by the surface expression of TCR $\beta$, Qa-2 and CD62L (data not shown), which also indicate that GFI1B-transgenic mice have greater numbers of phenotypically mature CD4 SP thymocytes. We have also examined the effects of the GFI1B transgene on a class II MHC null background to find that no CD4 SP cells were generated (data not shown). These data exclude the possibility that the increased CD4 SP cells are due to redirection of MHC-class-I-restricted CD8 SP development into the CD4 lineage. Altered signaling during activation in GFI1B transgenic thymocytes may lead to the accumulation of cells which should not normally 
be selected (defective negative selection), or the accumulation of cells unable to mature and egress.

The development of CD8 SP T cells in GFIB transgenic mice is not rescued by expression of the HY Class I restricted transgene.

It is unlikely that GFI1B represses CD8 or MHC class I expression since thymocytes from GFI1B-transgenic mice reveal normal surface expression of CD8 $\alpha$ and CD8 $\beta$ in the DP fraction, and are not Class I-deficient (data not shown). To determine if the GFI1B block to CD8 development involved an alteration in TCR repertoire selection, GFI1B transgenic mice were mated to $\mathrm{RAG}^{-1 /} / \mathrm{HY}$ mice and resulting progeny were backcrossed to generate GFI1B/ RAG2 $2^{-/} / \mathrm{HY}$ mice. The HY transgene encodes a Class Irestricted TCR that selects large numbers of $\mathrm{V} \beta 8^{+}$thymocytes into the $\mathrm{CD} 8^{+} \mathrm{T}$ cell lineage in female mice (42). Because the Rag2 gene product is necessary for TCR rearrangement, all RAG2 $2^{-/ /} / \mathrm{HY} / \mathrm{GFI} 1 \mathrm{~B}$ transgenic thymocytes express only the HY TCR as evidenced by V $\beta 8$ staining (data not shown). Positive selection of $C D 8^{+} \mathrm{T}$ cells by the HY TCR was severely reduced in the RAG2 $2^{-/ / H Y / G F I 1 B ~ f e m a l e ~ m i c e ~ a s ~ c o m p a r e d ~ t o ~}$ controls (Figure 11). Therefore, the critical defect in GFI1B transgenic mice is not simply an inability to form a class I restricted TCR.

\section{GFI1B expression overcomes a block to DP development imposed by autoreactive}

\section{TCR signaling.}

To determine if GFI1B-transgenic thymocytes display defective TCR signaling and/or activation in vivo we next examined the male $\mathrm{RAG} 2^{-/} / \mathrm{HY} / \mathrm{GFI} 1 \mathrm{~B}$ mouse thymus. The HY TCR recognizes the male HY antigen when presented by H2-D ${ }^{b}$. Male HY transgenic thymocytes are blocked at the DN stage by auto-reactive HY TCR signaling 
Figure 11. Expression of GFI1B inhibits the development of female HY-transgenic CD8 SP T cells. Thymocytes from 4 week-old female RAG2 $2^{-/} / \mathrm{HY}$ and RAG2 $2^{-/-}$ /HY/GFI1B mice were examined for the expression of CD4, CD8, and TCR V $\beta 8$ (utilized in the HY transgene) by flow cytometry. Representative FACS plots are shown with the total number of RAG2 $2^{-/} / \mathrm{HY}$ and RAG2 $2^{-/} / \mathrm{HY} / \mathrm{GFI} 1 \mathrm{~B}$ thymocytes above the plots. Thymocyte subsets are expressed as absolute cell numbers X $10^{6}$ per thymus ${ }^{ \pm}$ SEM. Note: These data were generated by Dr. Mary Kate Kitay. 
Figure 11
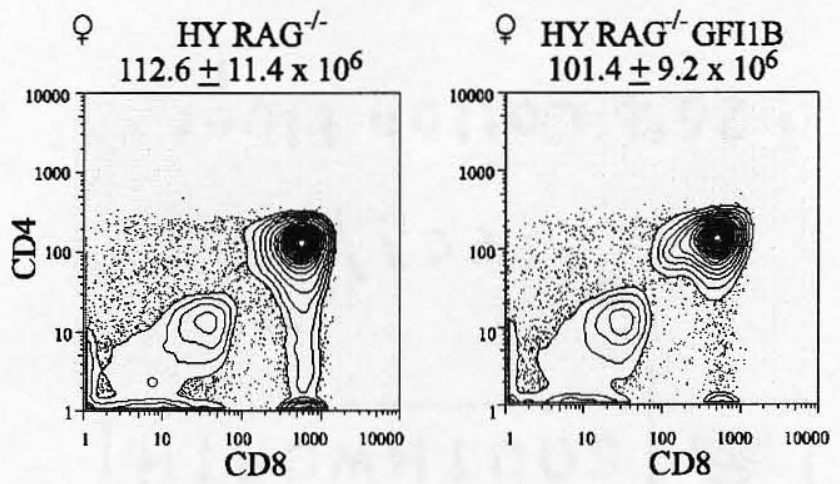

\begin{tabular}{|lcr|}
\hline Thymocyte Subset & $\begin{array}{c}\text { WT } \\
(\mathrm{n}=2)\end{array}$ & $\begin{array}{c}\text { GFI1B } \\
(\mathbf{n}=4)\end{array}$ \\
\hline DN & $9.43 \pm 5.1$ & $10.97 \pm 2.0$ \\
DP & $69.79 \pm 11.1$ & $64.53 \pm 7.5$ \\
CD4 SP & $0.08 \pm 0.0$ & $0.67 \pm 0.1$ \\
CD8 SP & $8.42 \pm 1.0$ & $1.05 \pm 0.1$ \\
\hline
\end{tabular}


that mimics negative selection (43). Mutant mice with defective intracellular signaling overcome this block and accumulate V $\beta 8^{+} \mathrm{DP}$ cells (44-46). GFI1B-transgenic peripheral $\mathrm{T}$ cells are defective in T-cell activation (Figures 8D and 9A). In agreement with these data, GFI1B-transgenic thymocytes overcome the block to development imposed by the autoreactive HY transgene, as shown by a modest four-fold accumulation of $\mathrm{CD}^{+} \mathrm{TCR} \beta^{+}$cells $(11.1 \%$ versus $47.9 \%)$ and a doubling of total thymus cellularity (Figure 12A and 12B). Therefore, our in vitro and in vivo data support a model in which ectopic expression of GFI1B leads to defective T cell activation.

\section{GFI1B impairs CD8 SP development in vitro, and decreases IL-7Ra expression.}

We have previously described an in vitro model system in which DP thymocytes can be signaled to differentiate into CD8 SP T cells (37). In this experimental system, signaled DP thymocytes initially terminate CD8 transcription, differentiate into $\mathrm{CD} 4^{+} 8^{-}$ intermediate thymocytes, and upregulate surface expression of IL-7R. In the presence of IL-7, $\mathrm{CD}^{+} 8^{-}$intermediate thymocytes terminate CD4 transcription and re-initiate CD8 transcription (events referred to as 'coreceptor reversal') and ultimately differentiate into CD8 SP T cells (37). Consequently, we assessed the ability of signaled DP thymocytes from wildtype (WT) and GFI1B transgenic mice to differentiate in vitro into CD8 SP T cells (Figure 13A).

We isolated WT and GFI1B transgenic DP thymocytes (Figure 13A, $\mathrm{D}_{0}$ ) and stimulated them with phorbal-12-myristate-13-acetate (PMA) and ionomycin (P+I) as previously described (37). Signaled DP thymocytes from both WT and GFI1B transgenic mice were induced to differentiate into $\mathrm{CD}^{+} 8^{-}$intermediate cells (Figure 13A, D2). Notably, in vitro generated intermediate $\mathrm{CD}^{+} 8^{-}$cells from GFI1B transgenic mice 
Figure 12. Expression of GFI1B overcomes a block to development in male HYtransgenic T cells. Representative FACS plots showing expression of CD4 and CD8 (A) or CD8 and TCR V $\beta 8$ (B) in male HY transgenic mice. Male RAG2 $2^{-/} / \mathrm{HY} / \mathrm{GFI} 1 \mathrm{~B}$ mice show a 4-fold increase in the number of $\mathrm{CD} 4^{+} \mathrm{CD} 8^{+}$and TCR $\mathrm{V} \beta 8^{+}$thymocytes when compared to RAG2 $2^{-/}$HY littermates. The total numbers of RAG2 $2^{-/} / \mathrm{HY}$ and RAG2 $2^{-/-}$ /HY/GFI1B thymocytes are shown above the plots. Note: These data were generated by Dr. Mary Kate Kitay and re-analyzed by Loretta Doan. 
Figure 12
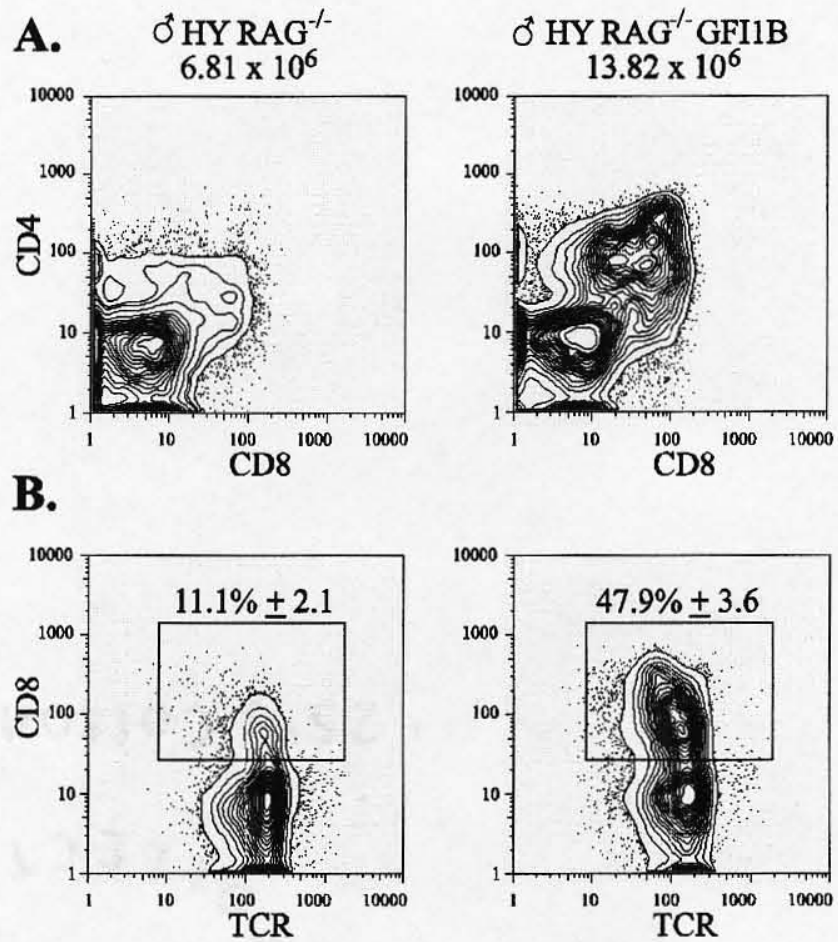
Figure 13. GFI1B impairs co-receptor reversal. A. (D0) Purified $\mathrm{CD} 4^{+} \mathrm{CD} 8^{+}$cells were obtained from pooled thymocytes of four 4-week-old GFI1B transgenic or four control littermates by panning with anti-CD8 antibody, and subsequently stimulated with PMA and ionomycin $(\mathrm{P}+\mathrm{I})$ for $16 \mathrm{hrs}$ at $37^{\circ} \mathrm{C}$. Stimulated cells were stripped of their surface coreceptors with pronase protease, cultured in complete media overnight, stained for $\mathrm{CD} 4$ and $\mathrm{CD} 8$ expression and purified $\mathrm{CD}^{+} 8^{-}$cells were obtained by cell sorting. (D2) Control (dashed line) and GFI1B transgenic (solid line) cells were analyzed by flow cytometry for IL-7R $\alpha$ expression. Purified $\mathrm{CD}^{+} 8^{-}$cells $\left(2 \times 10^{6}\right)$ were cultured overnight in the presence of IL-7. Flow analysis of samples of each culture indicated CD4 and CD8 surface levels before (D3) and after pronase protease treatment and culture in complete media overnight (D4). Note: These data were generated by Dr. Qing Yu and Dr. Alfred Singer (Experimental Immunology Branch, National Cancer Institute, National Institutes of Health, Bethesda, MD 20892). B. Four week old GFI1Btransgenic (solid line) and normal littermate control (dashed line) thymocytes were stained with antibodies against CD4, CD8 and IL-7R $\alpha$ and analyzed by flow cytometry. Representative histograms comparing the IL-7R $\alpha$ expression in thymocyte subpopulations are shown. Note: These data were generated jointly by Loretta Doan and Dr. Mary Kate Kitay. 
Figure 13
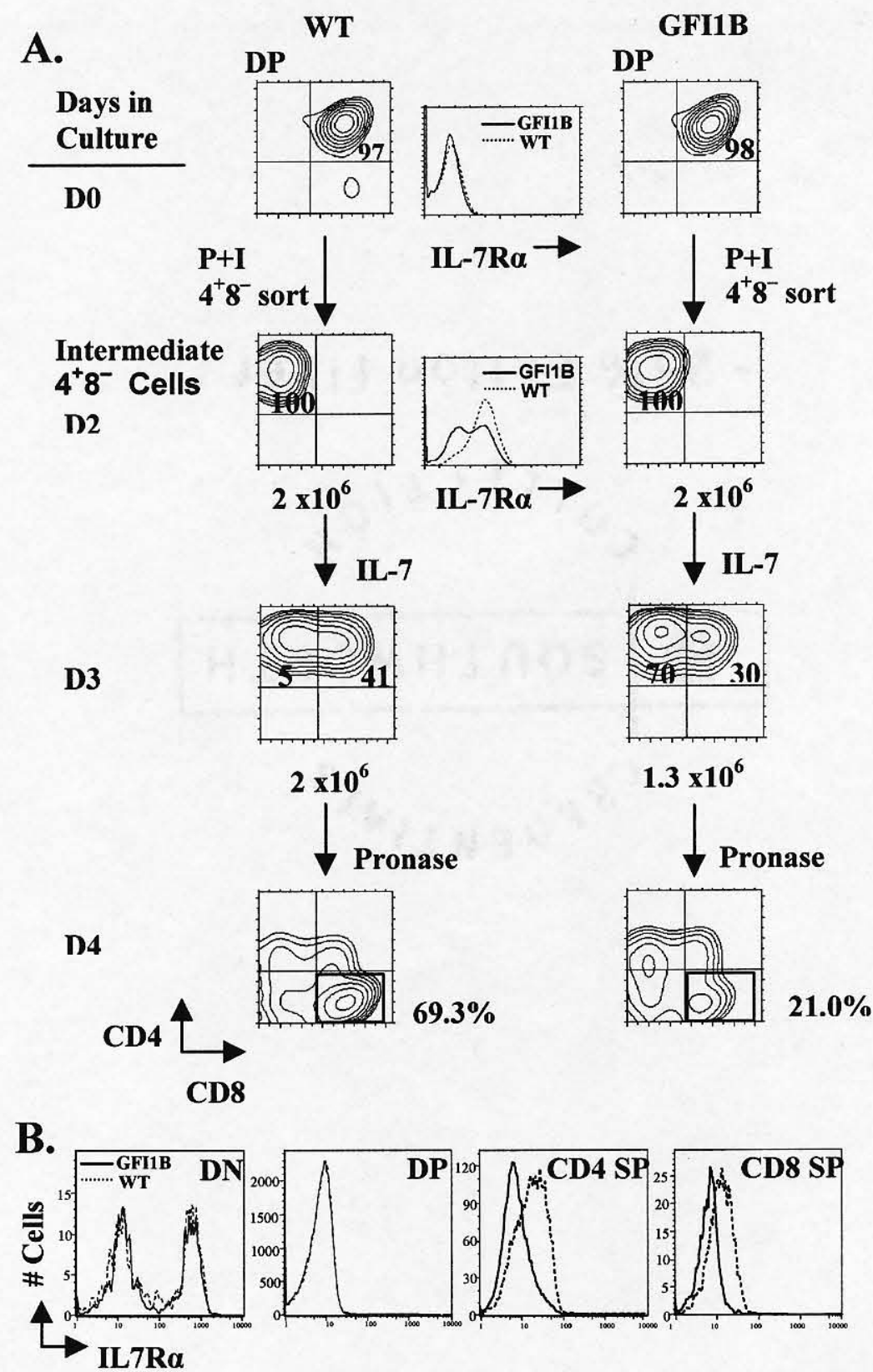
expressed lower surface levels of IL-7R $\alpha$ compared to cells from WT mice (Figure 13A, D2). We then added IL-7 to both populations of in vitro generated intermediate $\mathrm{CD}^{+} 8^{-}$ thymocytes, and, after $24 \mathrm{~h}$, pronase stripped the cells to remove pre-existing CD4/CD8 surface proteins so that we could determine the $\mathrm{CD} 4 / \mathrm{CD} 8$ proteins that the cells were actively synthesizing. Addition of IL-7 (Figure 13A, D3) followed by pronase stripping and re-expression culture revealed that $69.3 \%$ of WT cells had undergone coreceptor reversal and differentiated into CD8 SP T cells. In contrast, only $21.0 \%$ of GFI1B transgenic cells had undergone coreceptor reversal to become CD8 SP T cells (Figure 13A, D4). Thus, GFI1B transgenic thymocytes at the $\mathrm{CD}^{+} 8^{-}$intermediate stage of development are quantitatively deficient in their ability to undergo coreceptor reversal in response to IL-7 and are impaired in their ability to undergo in vitro differentiation into CD8 SP T cells.

To determine if the lower in vitro expression pattern of IL-7R $\alpha$ on GFI1B transgenic thymocytes was a possible explanation for the defective CD8 SP development in vivo, we next examined the expression of IL-7R $\alpha$ in unmanipulated thymocytes. Cells were stained for CD4, CD8 and IL-7R $\alpha$, then gated on thymocyte subpopulations and analyzed for IL-7R $\alpha$ expression (Figure 13B). The level of IL-7R $\alpha$ on DN thymocytes was not altered by the presence of the GFI1B transgene. However in both CD4 SP and CD8 SP thymocytes, in which IL-7R $\alpha$ expression is regulated by TCR signaling, GFI1B transgenic mice had lower levels of IL-7R $\alpha$ than wild type littermate controls (Figure 13B). Thus, GFI1B transgenic thymocytes are defective in both $T$ cell activation and the expression of activation-induced genes such as CD25, CD69 and IL-7R $\alpha$. The lower level of IL-7R $\alpha$ on GFI1B transgenic thymocytes provides a potential explanation for 
altered CD8 SP development in the GFI1B transgenic mice. Moreover, examination of peripheral $T$ cell subsets revealed lower levels of IL-7R $\alpha$ (data not shown). Since IL-7 has been shown to regulate the survival of naïve CD4 T cells (47-50) the lack of IL-7R $\alpha$ expression is a possible explanation for peripheral CD4 T cell lymphopenia.

\section{Bi-transgenic BCL2/GFI1B mice generate CD8 SP thymocytes.}

GFI1B-transgenic thymocytes express lower levels of IL-7R $\alpha$ (Figure 13B). IL-7 maintains the expression of endogenous BCL2 in T-lineage cells (51). BCL2 does not support thymic positive selection in the absence of MHC (52-54); however, transgenic expression of BCL2 can substitute for survival signals induced by cytokines such as IL-7 (54). GFI1B-transgenic mice were mated to E $\mu$-BCL2-25 transgenic mice, in which the human BCL2 transgene is expressed mainly in T lineage cells (33). As expected by the presence of the BCL2 transgene, total thymocyte cellularity was increased in bitransgenic mice (33); however, we found that BCL2/GFI1B bi-transgenics show increased numbers of $\mathrm{TCR}^{\text {int-hi }} \mathrm{CD} 8 \mathrm{SP}$ thymocytes and a normal ratio of CD4 SP to CD8 SP thymocytes (Figure 14). While BCL2 may have pleiotropic effects on T-cell development (53), the ability of BCL2 to rescue CD8 SP development in GFI1Btransgenic mice is consistent with the role of BCL2 as a downstream target of IL-7.

\section{Bi-transgenic GFI1/GFI1B mice generate CD8 SP thymocytes.}

We next determined whether GFI1 could alter the defects engendered by GFI1B expression. GFIl enhances T cell activation whereas GFI1B impairs this process (Figure 8). Since GFI1 and GFI1B bind to the same DNA sequence, it is possible that some of the defects in GFI1B transgenic thymocytes are the result of an imbalance between DNAbound GFI1B versus GFI1. In fact, six-week-old GFI1/GFI1B bi-transgenic mice 


\section{Figure 14. Transgenic BCL2 restores CD8 SP thymocytes in GFI1B transgenic}

mice. Thymocytes from GFI1B and BCL2/GFI1B bitransgenic littermates were stained with antibodies to TCR $\beta, \mathrm{CD} 4$, and CD8, and analyzed by flow cytometry. Cells are gated on TCR ${ }^{\text {int-hi }}$ events, and representative CD4 versus CD8 FACS profiles are depicted. A scatter plot of CD8 SP cells in BCL2-transgenic, GFI1B-transgenic, and BCL2/GFI1B-bitransgenic mice reveals the increase in the CD8 SP population in the bitransgenic animals (in comparison to GFI1B-transgenic mice). Each diamond represents the percent of $\mathrm{TCR}^{\text {int-hi }} \mathrm{CD} 8 \mathrm{SP}$ cells per thymus of an individual mouse (5-7 mice/group). The black bar is the mean value for each group. Note: These data were generated by Dr. Mary Kate Kitay and re-analyzed by Loretta Doan. 


\section{Figure 14}
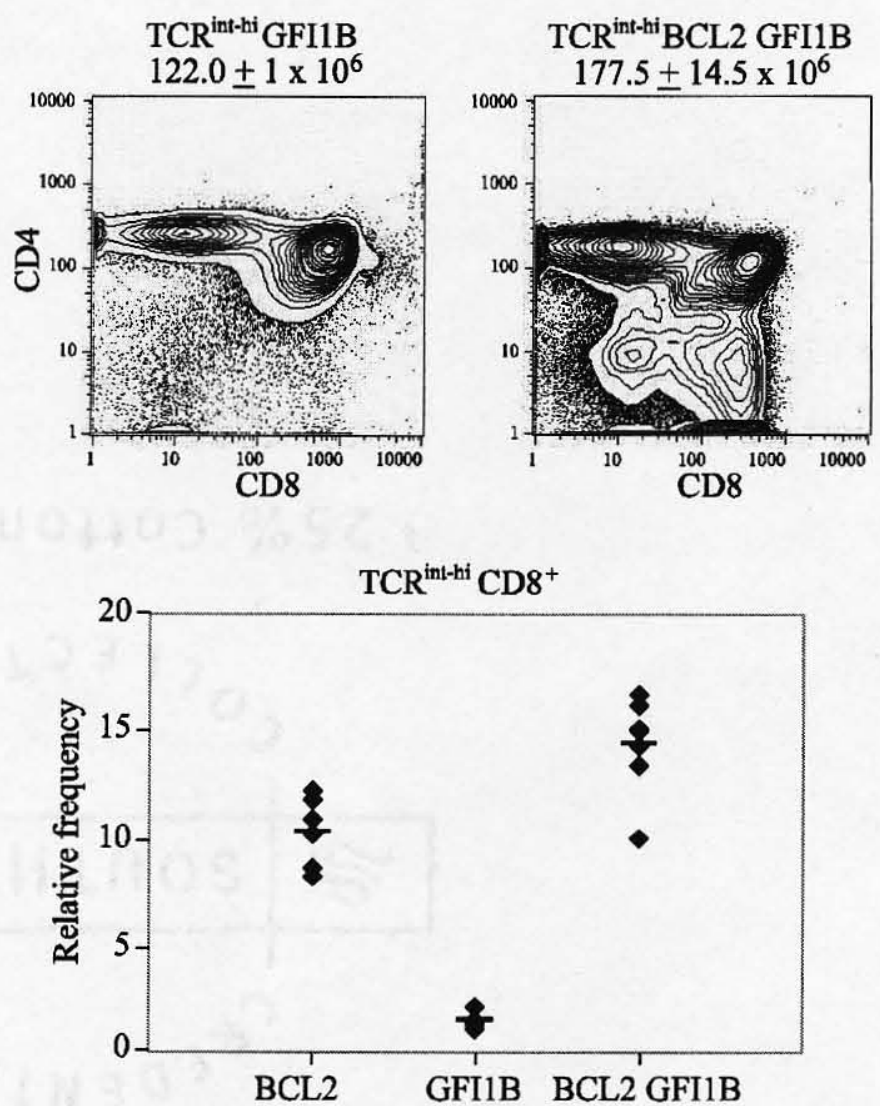
Figure 15. Transgenic GFI1 increases the generation of CD8 SP thymocytes in GFI1B transgenic mice. Thymocytes from 6-week-old GFI1B and GFI1/GFI1B bitransgenic littermates were stained with antibodies to TCR $\beta, C D 4$, and CD8, and analyzed by flow cytometry. Cells are gated on $T C R^{\text {int-hi }}$ events, and representative CD4 versus CD8 FACS profiles are depicted. A scatter plot of CD8 SP cells in GFI1transgenic, GFI1B-transgenic, and GFI1/GFI1B-bitransgenic mice shows the partial restoration of CD8 SP thymocytes observed in bi-transgenic animals (in comparison to GFI1B-transgenic mice). Each diamond represents the percent of TCR ${ }^{\text {int-hi }}$ CD8 SP cells per thymus of an individual mouse (4-6 mice/group). The black bar is the mean value for each group. The increase is statistically significant with a $P$ value of 0.0003 as determined using a two-tailed Student's T test. 
Figure 15
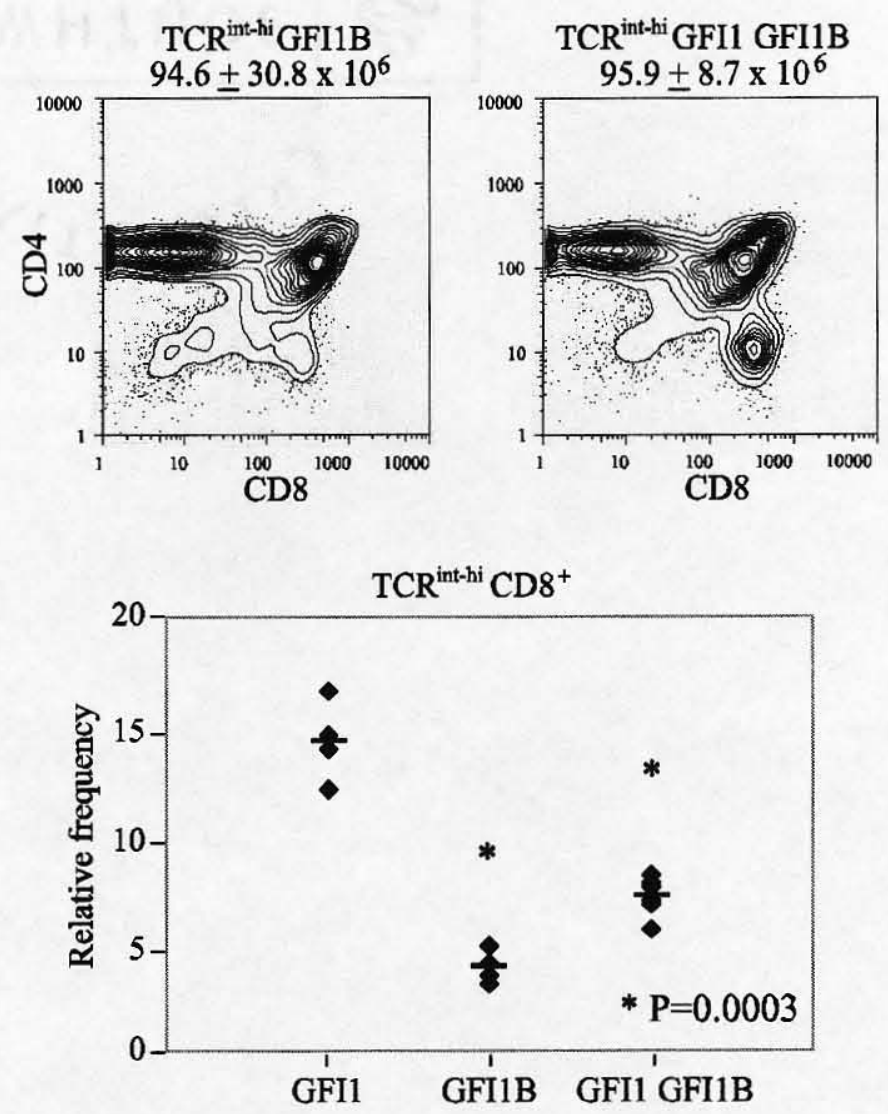
generate twice as many TCR ${ }^{\text {int-hi }}$ CD8 SP thymocytes than do littermate GFI1B

transgenics (Figure 15, $\mathrm{P}=0.0003$ ). The lower level of expression of the GFI1 transgene in comparison to the GFI1B transgene may explain the modest ability of GFI1 transgene to compete with transgenic GFI1B. Nevertheless, GFI1 expression increases the number of GFI1B-transgenic CD8 SP cells.

Transgenic expression of GFI1 does not alter other GFI1B-induced defects. The GFI1B-induced abnormal generation of phenotypically-mature CD4 SP thymocytes remains in the GFI1/GFI1B bi-transgenic thymus. Moreover, peripheral T lymphopenia in GFI1B transgenics is not altered by BCL2 or GFI1. It is known that BCL2-transgeneinduced elevation in thymocyte numbers does not alter peripheral $\mathrm{T}$ cell numbers (51). Likewise, we find that neither BCL2 nor GFI1 increases the number of peripheral CD8 SP T cells in the GFI1B-transgenic spleen. GFI1, like BCL2, appears to ameliorate only the GFI1B effect on the production of CD8 SP cells. The inability of either the BCL2 or GFI1 transgene to cancel the GFI1B-induced alteration in the expression of IL-7R $\alpha$ (data not shown) indicate that both BCL2 and GFI1 must act to increase CD8 SP generation by a mechanism that is independent of the induction of IL-7 signaling. However, it is formally possible that GFI1, like BCL2, acts downstream of IL-7 signaling and that transgenic expression of either protein mimics the effects of IL-7.

\section{Discussion.}

The phenotypes observed in GFI1 and GFI1B transgenic mice can be understood in the context of the normal expression pattern of either factor. GFI1 is induced at the transition between DP and SP cells. GFI1 may thus play a role in the activation-induced 
developmental steps between DP and SP thymocytes. GFI1B is induced at steps in thymocyte development in which thymocytes are activated. In fact, $\mathrm{RAG}^{-/}$cells that lack the ability to activate do not express GFI1B (Figure 6). GFI1 and GFI1B have opposite effects on $\mathrm{T}$ cell activation. Peripheral GFI1 transgenic $\mathrm{T}$ cells activate better than do those from wild type littermates, whereas GFI1B transgenic T cells are impaired in activation. Both phenotypes are cell autonomous as they occur in purified $\mathrm{T}$ cells from either transgenic (Figure 9 and data not shown).

The impairment of $\mathrm{T}$ cell activation function in GFI1B transgenic $\mathrm{T}$ cells appears to be linked to an inability to signal properly after TCR engagement and costimulation. First, purified GFI1B-transgenic T cells do not activate given CD28- and CD3crosslinking antibodies (Figure 9A). Second, the TCR-signaling-dependent RAS/MAPKinduced expression of early activation markers CD25 and CD69 (55) is impaired in GFI1B transgenic T cells (Figure 9B). Finally, in male HY transgenic mice GFI1B expression rescues the generation of DP thymocytes that are normally deleted due to strong autoreactive TCR signals (43). Similar results have been obtained in male HY transgenic mice that are rendered defective in TCR signaling by deletion of intracellular proteins that participate in the TCR-signaling cascade (44-46). However, the GFI1Binduced defect in $\mathrm{T}$ cell activation cannot be restricted to proximal signaling molecules in the TCR- and costimulatory-signaling pathways, since GFI1B-induced impairment of CD8 SP cell formation is also observed after in vitro drug-stimulated activation that bypasses the need for proximal TCR- and costimulatory-signaling events (Figure 13). The ability of GFI1B-transgenic thymocytes to respond to cytokine signaling may also be affected. Though activated GFI1B transgenic thymocytes fail to induce IL-7R $\alpha$ 
expression, transgenic expression of IL-7R $\alpha$ (56) did not change the GFI1B induced phenotypes (data not shown), whereas expression of the IL-7 downstream effector BCL2 increased CD8 generation. Thus, GFI1B expression in T cells induces cell autonomous defects in intracellular signaling that may impair $\mathrm{T}$ cell activation at multiple steps.

GFI1B may serve as a negative regulator of GFI1-enhanced activation. Both GFI1 and GFI1B are expected to repress genes to alter the kinetics or activation potential of intracellular signals. The induction of GFI1B in activated thymocytes could result in competition between GFI1 and GFI1B for DNA binding at specific promoters.

Alternatively, GFI1 and GFI1B may regulate different promoters. Both scenarios appear to be relevant to the GFI1B-induced T cell defects. Since transgenic GFI1 expression doubles the generation of GFI1B-transgenic CD8 SP cells, GFI1 and GFI1B may compete at promoters to regulate genes important for CD8 development. Target genes responsible for this phenotype may be properly regulated by GFI1, but improperly regulated by GFI1B in cells about to undergo lineage commitment. Interestingly, transgenic BCL2 or GFI1 rescued CD8 SP development, but did not affect GFI1Binduced changes in CD4 SP development, the expression of IL-7R $\alpha$ or peripheral T lymphopenia. Target genes responsible for the latter GFI1B-induced phenotypes should be independent of GFI1 regulation and instead uniquely regulated by GFI1B.

GFI1 and GFI1B differ in amino acid sequence in the region between the SNAG repressor domain and the zinc-finger DNA-binding domain. These dissimilar regions may mediate interaction with other proteins (such as other transcription factors or adapter proteins), leading to differential regulation of target promoters by GFI1 and GFI1B. The normal role of GFI1B in the thymus may be to regulate the extent of GFI1-mediated 
thymocyte activation during development. Our data are the first to demonstrate that GFI1 and GFI1B are not redundant for $\mathrm{T}$ cell activation functions, providing a potential biological explanation for the termination of GFI1B expression in mature T cells.

IL-7 is critical for the survival and proliferation of immature thymocytes into mature CD8 SP cells $(37 ; 54 ; 57)$. IL-7 is constitutively present in the thymus; however, IL-7R $\alpha$ expression is not induced until TCR stimulation ceases (37). Thus, whether TCR signaling continues or ceases determines the ability to differentiate into CD8 SP T cells. This new perspective on lineage commitment is referred to as the "kinetic signaling" model. In accordance with this model, GFI1B-transgenic thymocytes, in which GFI1 function is compromised, fail to integrate activation signals, resulting in impaired expression of activation-induced survival genes such as IL-7R $\alpha$ (37).

Models of lineage commitment which require quantitative or temporal differences in TCR signals to direct $\mathrm{T}$ cell development $(58 ; 59)$ would predict that the reduced activation in GFI1B-transgenic T cells should lead to improved CD8 SP development (60). Transgenic expression of GFI1B in the thymus instead results in a severe decrease in the generation of CD8 SP cells. A similar phenotype is exhibited by mice made deficient for interferon $\alpha, \beta$ signaling by knocking out the transcription factor IRF-1 (61). These mice are devoid of CD8 SP T cells even though they contain normal numbers of CD4 SP T cells. We have established that the GFI1B-transgenic thymus defects are not due to GFI1B repression of IRF-1 (Figure 7C), CD8 or MHC Class I (data not shown), the absence of a functional TCR rearrangement (Figure 11) or to a TCR-signalindependent redirection of $\mathrm{CD} 8$ cells to a CD4 lineage choice (data not shown). However, in agreement with the kinetic signaling model, a signal-independent survival 
cue from the downstream target of IL-7, BCL2, increases CD8 SP generation in the context of GFI1B expression. Therefore, the defect in generation of CD8 SP cells in GFI1B-transgenic mice appears to be linked to an inability to transduce a post-activation signal-dependent survival cue.

GFI1 functions to inhibit T cell death induced by specific stimuli; GFI1 increases survival of explanted thymocytes (62) and decreases apoptosis induced by TCR ligation (21). In GFI1B transgenic T cells, GFI1 expression does not correct GFI1B-impaired expression of IL-7R $\alpha$; however, like BCL2, transgenic GFI1 increases CD8 SP generation in the GFI1B transgenic thymus. The salient function of GFI1 in CD8 SP generation may be GFI1 mediation of cytokine induced survival signaling that is impeded in GFI1B transgenic thymocytes. Taken together, these data are the first to suggest a role for GFI1 in the integration of activation signals from the TCR with survival signals from cytokines such as IL-7. 


\section{CHAPTER IV}

\section{GFI1 IS AUTOREGULATORY IN T CELLS, BUT NOT IN A MYELOID \\ LINEAGE CELL LINE}

\section{Introduction.}

Growth Factor Independence $1(G f i l)$ and GfilB are two closely related oncogenes that play different but pivotal roles in hematopoiesis. Gfil deficient mice display both thymic and peripheral $\mathrm{T}$ lymphopenia, with severe abnormalities in pre- $\mathrm{T}$ cell development $(11 ; 16 ; 17)$. Furthermore, they display a profound defect in neutrophil differentiation, which leads to neutropenia $(16 ; 17) . G f i l B$ is necessary for the development of megakaryocytes and for definitive erythropoiesis (18). Deficiency of GfilB is embryonic lethal by day E15 (18). Given embryonic lethality, no thymic or T cell phenotype has been reported for GfilB deficiency; however, GFI1B transgenic mice display perturbed $\mathrm{T}$ cell development and function (19). In accordance with the distinct hematopoietic phenotypes of $G f i l$ and $G f i l B$ deficiency, normal adult animals express these two factors in a tissue-specific manner (10). Gfil is predominantly expressed in thymus, the site of $\mathrm{T}$ cell development, while GfilB is expressed in spleen. Both factors are expressed in bone marrow (10).

The unique physiological functions of $G f i l$ and $G f i 1 B$ may seem somewhat surprising given the similarities of their defined biochemical and oncogenic 
functions $(9 ; 10)$. GFI1 and GFI1B are two members of a family of zinc-finger transcriptional repressors that are characterized by the presence of the SNAG (found in the Snail and Gfil family of proteins) repression domain (9). Transcriptional repression by both GFI1 and GFI1B requires an intact SNAG domain. Specifically, in in vitro transient transcription assays, SNAG activity can be abrogated by mutation of the Proline at position 2 to Alanine (P2A) (9). Furthermore, GFI1 and GFI1B have been shown to bind very similar consensus DNA sequences, both of which have an absolute requirement for the tetranucleotide sequence AATC (10). Mutation of this AATC core to GGTC results in a lack of DNA binding in vitro (15). Despite the extensive similarities of these proteins, each contains a region unique in amino acid sequence, the activities of which are yet to be defined.

We and others have reported that in T cells, transgenic expression of GFI1 causes an increase in the response to TCR-stimulated activation (19-21). Conversely, we have shown that GFI1B is induced during T lymphopoiesis at stages involving activation, and that transgenic expression of GFI1B in T cells leads to decreased activation (19). These effects were seen in both mature T cells in vitro and in thymocytes in vivo. While GFI1B transgenic mice display normal thymic cellularity, they share with Gfil deficient mice the characteristic of peripheral T lymphopenia. In addition, GFI1B transgenics display thymic abnormalities, including a defect in the formation of CD8 SP cells, which can be partially corrected by transgenic expression of GFI1(19). Thus, some of the observed GFI1B-mediated effects on T lymphopoiesis may result from the disruption of normal functions of GFI1 (19). We therefore sought to determine the mechanism by which GFI1B interferes with GFI1 activity. 
Upon comparison of expression levels of the transgenic GFI1 and GFI1B, we discovered that endogenous GFI1 is not expressed at detectable levels in GFI1B transgenic thymocytes. Given the roles of GFI1 and GFI1B as transcriptional repressors, we hypothesized that transgenic GFI1B represses the transcription of endogenous Gfil. In this report, we show that mouse, rat, and human Gfil are repressed by both GFI1 and GFI1B, and that this repression is both direct and dependent on the SNAG repressor domain in vitro and in vivo. In addition, we identify in vitro GFI1 binding of consensus recognition sequences in regulatory regions of the Gfil gene, and suggest previously undefined sequence requirements for binding by GFI1 in nuclear complexes. Furthermore, we provide evidence that GFI1 autoregulation may be a cell-type-specific mechanism, occurring in primary $\mathrm{T}$ cells and a $\mathrm{T}$ cell line, but not in a myeloid lineage cell line.

\section{Materials and methods.}

Mice. The GFI1 and GFI1B transgenic mice were previously described (19). P2A-GFI1 transgenic mice were generated similarly by cloning the P2A-GFI1-mutant cDNA into the BamHI site of the TLC vector (32). All mice were housed in the Donald Baxter Barrier Facility at the University of Louisville and used in accordance with protocols approved by the U of $L$ Institutional Animal Care and Use Committee.

Plasmids. Plasmids used in transient transcription assays were previously described (9). Plasmids for in vitro transcription and translation were generated by cloning rat Gfil, $\triangle Z n 4 G f i 1$, or mouse GfilB into the pcDNA3 vector (Invitrogen). Retroviral vector 
expression constructs were generated by cloning triple Flag-epitope-tagged GFI1 and mutant GFI1 (SV40swap) (9) into the MIEV vector (63).

Transfections, transduction, and transcription assays. For stable transfections, Jurkat cells were electroporated as previously described (9). TLC transgene constructs encoding GFI1 or GFI1B were co-transfected with empty pcDNA3.1 vector DNA at a ratio of 22:1. Transfected cells were selected in the presence of $1 \mathrm{mg} / \mathrm{mL}$ Geneticin (Invitrogen) and cloned by limiting dilution.

293T cells were maintained in Dulbecco's Modified Eagle's Medium (DMEM) with 10\% FBS, 1\% L-Gln, 1\% Pen/Strep (Invitrogen). Cells were transfected with Lipofectamine 2000 (Invitrogen) according to the manufacturer's protocol using OptiMEM low serum medium (Invitrogen) and $2.5 \times 10^{5}$ cells/well in 24-well plates that were pre-coated with poly-L-Lysine $(0.1 \mathrm{mg} / \mathrm{mL}$, Sigma). Transfections were performed in triplicate. CAT assays were performed as previously described (9). A 2-tailed Student's $\mathrm{T}$ test determined $\mathrm{P}$ values.

For transduction experiments, Phoenix cells (64) were transiently transfected with retroviral constructs using calcium phosphate $\left(\mathrm{Ca}_{3}\left(\mathrm{PO}_{4}\right)_{2}\right)(65)$ then co-cultured with U937 cells overnight. The transduced U937 cells were expanded and sorted on a FACSVantage (BD); sorted GFP ${ }^{+}$cells were analyzed after two weeks in culture.

Northern and Western blot. For Northern blots, total RNA was extracted using Ultraspec RNA Isolation Solution (Biotecx Laboratories, Inc. Houston, TX) according to the manufacturer's protocol, and poly- $\mathrm{A}^{+} \mathrm{RNA}$ was obtained using Oligotex Direct

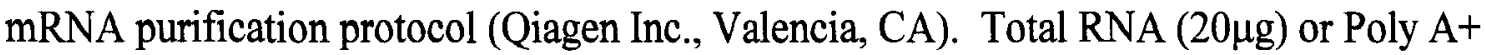
RNA $(5 \mu \mathrm{g})$ was electrophoresed in a $1 \%$ agarose-formaldehyde gel and transferred to 
MagnaGraph nylon membrane (Micron Separations, Inc., Westboro, MA). Membranes were probed in UltraHyb solution (Ambion, Austin, TX) according to the manufacturer's protocol. Radioactive probes were generated by Prime-1-Gene random priming kit (Promega, Madison, WI).

Western blot detection of GFI1 and GFI1B was performed as previously described (19). Nuclear extracts of U937 cells were made using NE-PER (Pierce) according to manufacturer's instruction, and protein concentration was determined using BCA Protein Assay Reagent (Pierce). Primary antibody was either a commercially available antiserum to the last 20 amino acids of GFI1 (Santa Cruz, sc-6357), anti-FlagHRP (Sigma), or a mouse monoclonal to GFI1 (2.5D.17).

RNase and DNase. For resolution of the sequence of the rat locus, primer extension was performed with an end-labeled oligonucleotide complementary to exon 1 sequence, hybridized to $50 \mu \mathrm{g}$ total RNA from rat Nb-2 lymphoma cells or normal rat thymus, and the reaction products were analyzed by electrophoresis through a $7 \%$ polyacrylamide/7M urea sequencing gel.

To characterize the exon-intron boundaries and genomic organization of $G f i 1$, an F344 rat genomic $\lambda$ Dash clone (8) was digested with EcoR1 and the resulting fragments subcloned in pBluescript SK (Stratagene, Valencia, CA). Next, oligonucleotide primers based on the Gfil cDNA sequence were used to probe Southern blots of the genomic subclones, for PCR amplification, and for sequencing.

Cultures were harvested, and nuclei were isolated and subjected to incremental DNase I digestions as previously described (66). 
Electrophoretic mobility shift assays (EMSA). Shifts were performed essentially as described (14). In vitro transcription and translation (IVT) was performed using TNT T7-coupled reticulocyte lysate system (Promega) according to the manufacturer's protocol. Jurkat nuclear extracts (NE) were prepared using a standard Dignam protocol (67). The sense sequence of the -705 probe is 5 '-

GGAGCAAACCTCAGGGATTGGGTGTCAAGGTA-3', and the mutant probe is 5'GGAGCAAACCTCAGGGACCGGGTGTCAAGGTA-3'. The underlined bases were added for labeling using Klenow (NEB) and $\alpha-{ }^{32} \mathrm{P}-\mathrm{dCTP}$ (Amersham, Piscataway, NJ). $\mathrm{NE}$ (2.5 ug ) or IVT protein ( 3 to $5 \mathrm{uL}$ ) was pre-incubated in binding buffer (14) at room temperature for 20 minutes, with cold oligonucleotide or antibodies [anti-GFI1 (sc-8558) or normal goat antiserum (sc-2028)] for competition and super-shift assays. Labeled probe $(50,000 \mathrm{cpm})$ was added, and the reaction continued at room temperature for 30 minutes. Samples were electrophoresed through a $6 \%$ non-denaturing polyacrylamide gel, which was dried and exposed to film.

ChIP. ChIP assays were performed as previously described (13). Each experiment was performed twice with similar results, and representative data are shown.

\section{Results.}

Endogenous murine $G$ fil is repressed by transgenic expression of GFI1 or GFI1B in primary thymocytes.

Transgenic expression of GFI1B engenders defects in T lymphopoiesis, some of which can be rescued by simultaneous forced expression of GFI1 (19). Because GFI1 and GFI1B have nearly identical DNA binding domains (10), we reasoned that GFI1B 
may alter T lymphopoiesis by competing with endogenous GFI1 for DNA binding on specific promoters. However, Western blot analysis of whole cell lysates from GFI1Btransgenic thymocytes revealed undetectable levels of GFIl protein (Figure 16A). These results led us to investigate the levels of endogenous Gfil transcript in GFI1 and GFI1Btransgenic thymocytes. Since the transgenes lack the Gfil 3' untranslated region, we performed Northern analysis with a murine Gfil 3'-untranslated-region probe on poly$\mathrm{A}^{+}$-selected RNA from GFI1B-transgenic thymi. These analyses revealed a dramatic reduction in the level of endogenous Gfil transcript (Figure 16B). Moreover, transgenic mice with forced low-level expression of GFI1 display a mild reduction of endogenous Gfi1 message (Figure 16B). Therefore, GFI1B-transgenic T cells are essentially devoid of GFI1, and Gfil may be targeted for repression by both GFI1 and GFI1B.

\section{The SNAG-repression-domain mutant P2A-GFI1 profoundly de-represses endogenous murine Gfil.}

The P2A-GFI1 mutation significantly impairs GFI1-mediated repression (9). Therefore, we predicted that the P2A-GFI1 mutant would act as a dominant-negative and interfere with GFI1 function. We constructed transgenic mice in which P2A-GFI1 is expressed in $\mathrm{T}$ cells through the $L c k$ proximal promoter and a $C D 2$ enhancer (Figure 16C) (32). Deletion of Gfil leads to a significant decrease in thymocyte numbers and impairs T lymphopoiesis $(11 ; 16 ; 17)$. However, contrary to our expectation of dominantnegative GFI1 activity by the P2A-GFI1 mutant, thymi of P2A-GFI1-transgenic mice display no discernable phenotype (data not shown). Northern analysis of thymus RNA with a transgene-vector-specific probe revealed adequate expression of $P 2 A-G f i 1$ 


\section{Figure 16. Endogenous murine $G f i I$ is repressed by GFI1 and GFIB in}

thymocytes. A. Western blot analysis of protein $(75 \mathrm{ug})$ from thymocyte whole cell lysates of two wild type (WT) and two GFI1B transgenic mice. The blots were probed with an antibody against the last 20 amino acids of GFI1 that is cross-reactive to GFI1B (sc-6375). A loading control (IRF1) is shown. B. Northern blot analysis of poly- $\mathrm{A}^{+}$ RNA (5 $\mu \mathrm{g})$. Endogenous Gfil was detected using a probe specific to the 3' untranslated region of mouse Gfil. Blots were stripped and probed with a loading control (MDM2). Note: These data were generated by Dr. H. Leighton Grimes and Loretta Doan. C. Diagram of the transgene construct used in creating P2A-GFI1 transgenic mice. The transgene vector(32) is identical to that utilized to create GFI1 and GFI1B transgenic animals. D. Northern blot analysis of total RNA (20 ug) from thymocytes of two wild type (WT) and two P2A-GFI1 (P2A) transgenic mice using the same probe as in B. Ethidium bromide staining is shown for equal RNA loading. 
Figure 16

A

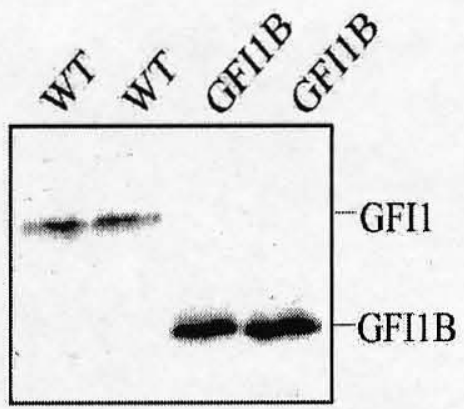

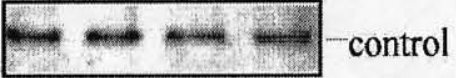

B
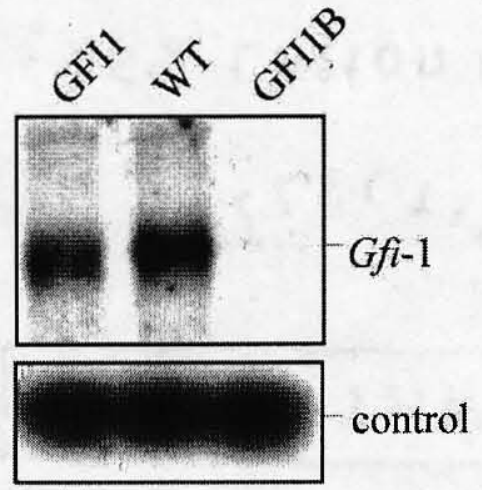

C polyA+ RNA

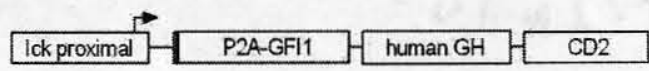

D
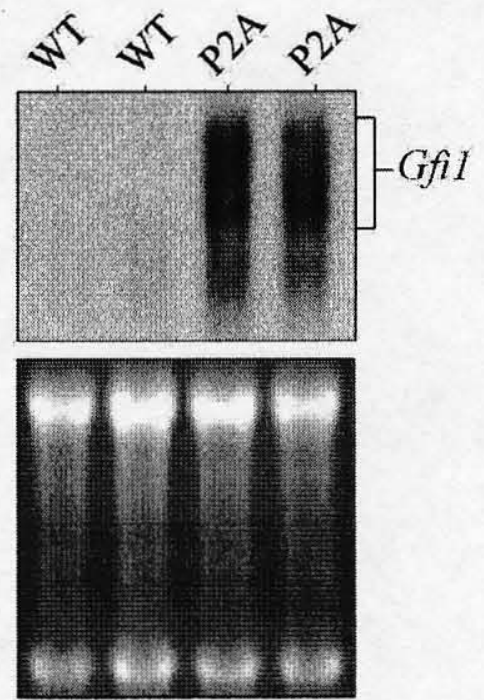

total RNA 
message (data not shown). We subsequently performed Northern analysis of P2Atransgenic thymocytes with the murine Gfil 3' untranslated region probe. This analysis revealed a striking de-repression of endogenous $G f i 1$ in the presence of transgenic P2AGFIl (Figure 16D). Whereas in normal thymocyte RNA the detection of Gfil requires poly- $\mathrm{A}^{+}$selection (Figure 16B), the Gfil signal in P2A-transgenic thymocyte RNA was easily detected by Northern blot analysis of $20 \mu \mathrm{g}$ of total RNA (Figure 16D). The profound de-repression of endogenous Gfil in repression-defective P2A-GFI1-transgenic thymocytes is evidence for direct autoregulation of Gfil.

\section{Identification of the rat $G f i 1$ promoter.}

Given the probability of direct repression of Gfil, we next wanted to examine the Gfil promoter for GFI1/GFI1B DNA-binding sites. However, the sequence of the Gfil promoter had not been well resolved. Early identification of Gfil as an oncogene was performed in rat $\mathrm{T}$ cell leukemia lines, providing a biological impetus for studying the transcriptional regulation of rat Gfil. Genomic clones from the rat locus were analyzed by restriction endonuclease digestion, followed by Southern blotting with Gfil cDNA probes and sequencing. We determined both the exon/intron boundaries of the gene (Figure 17A), and the organization of the rat Gfil locus (Figure 17B). Specifically, examination of the sequence of intron/exon boundaries revealed the presence of 7 exons and 6 introns. The exon/intron boundary sequences are homologous to the previously published mouse locus (68), with minor exceptions. The genomic organization of rat Gfil is diagramed in Figure 17B and spans $9.5 \mathrm{~kb}$ of sequence. These data were corroborated by the recently available rat genomic sequence in GenBank (data not shown). 


\section{Figure 17. Characterization of rat Gfil locus and identification of the transcription}

start site. A. Exon/intron boundaries of rat Gfil. Shown are the splice donor and acceptor sites as determined by sequencing. B. Schematic representation of the organization of the rat Gfil locus. Exons 1-7 are depicted as gray boxes. Arrows identify DNase hypersensitive sites, with the longest arrow representing the start site of transcription. Roman numerals indicate probes used for DNase hypersensitivity analysis. C. Southern blot analysis of DNase hypersensitivity assays. Nuclei from Nb2 (lanes 1-4 of left panel), A2 (lanes 5-8 of left panel), or LE3Spl (right panel) rat cell lines were digested with increasing concentrations of DNAse I, the DNA was digested with HindIII restriction endonuclease, and Southern blots were hybridized with probes I and II. $\lambda /$ HindIII molecular weight markers are indicated on the left of each blot. The arrow indicates the major site of hypersensitivity, corresponding to the longest arrow in panel B. Minor bands represent other regions of putative transcriptional control corresponding to smaller arrows in panel B. D. RNase protection assay identifies the start site of transcription. The left panel shows representative results of RNase protection analysis from rat $\mathrm{Nb}-2$ lymphoma cells (1) and normal rat thymus (2). Arrows indicate the major protected fragments and the corresponding nucleotides on a sequencing gel. The right panel depicts the sequence of the start site and the consensus sequence of an Inr. Note: All of these data were generated by Dr. C. Blake Gilks and Dr. Susan D. Porter (Department of Pathology and Genetic Pathology Evaluation Centre, Vancouver General Hospital and the University of British Columbia). 
Figure 17

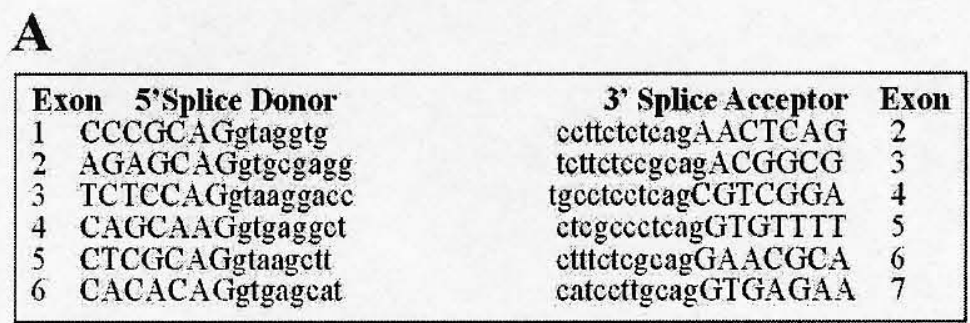
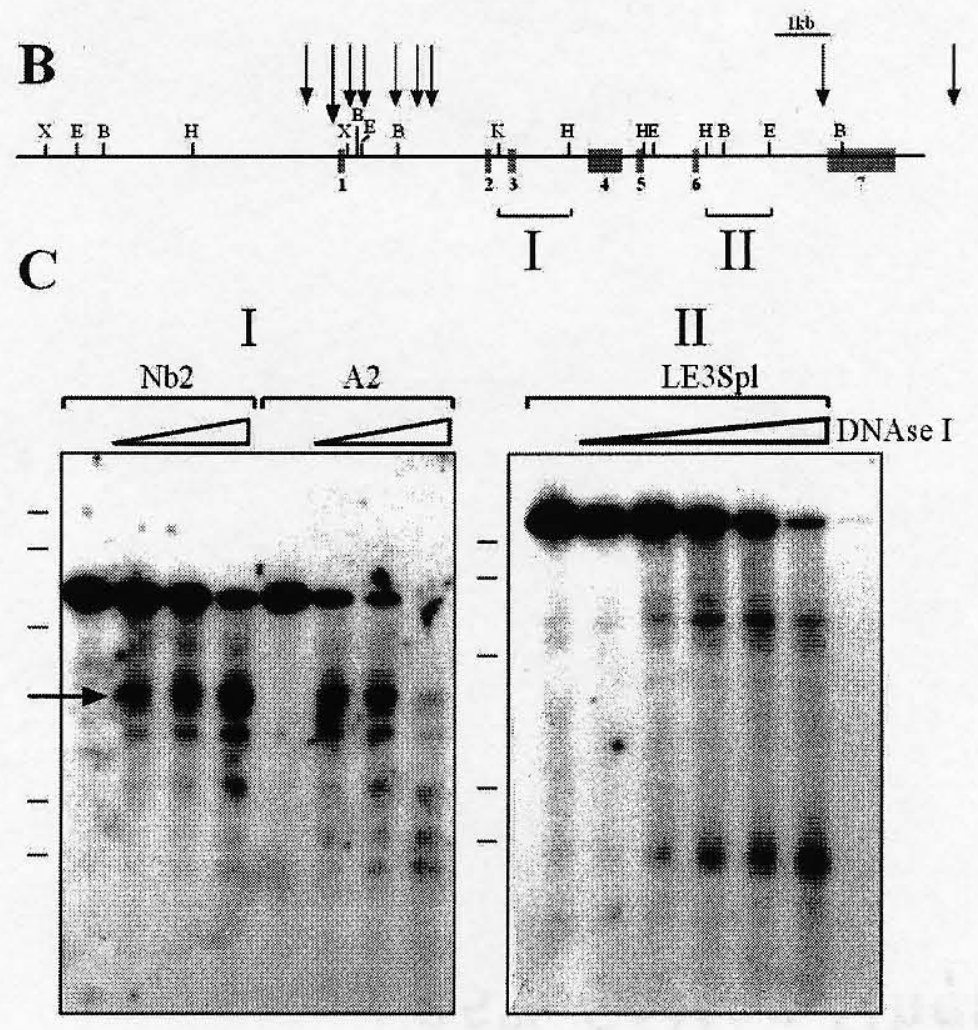

D

ATCG 12

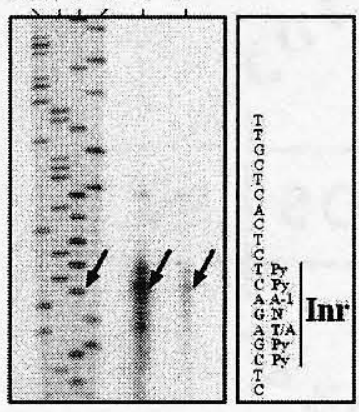


To determine potential regulatory regions, DNase hypersensitivity assays were performed (Figure 17C). Analysis of rat $\mathrm{Nb} 2$ and $\mathrm{A} 2$ lymphoma cells using a genomic probe (Figure 17B, I) revealed hypersensitive sites clustering around exon 1 (arrows in Figure 17B). Many of these sites occur within the first intron, but the major site of DNase hypersensitivity is just upstream of the first exon (longer arrow Figure 17B, and arrow Figure 17C). In LE3Spl cells, two downstream hypersensitive sites were also identified using probe II and are indicated in Figure 17B. The same pattern was seen with both probes with or without prolactin addition to prolactin-dependent $\mathrm{Nb} 2$ cells, and with or without Interleukin-2 addition to the Interleukin-2-dependent LE3Spl cell line (data not shown).

We next mapped the start site of transcription of the rat $G f i l$ gene. The mouse Gfil locus has both a major transcription start site 5 ' of exon 1a, and a minor one in the first intron (68). Therefore, we performed RNase protection analysis to locate the transcriptional start site(s) in the rat Gfil gene. In RNA from rat Nb2 lymphoma cells, a sequence proximal to exon 1 was identified as the single start site of transcription in the rat Gfil gene (Figure 17D). This sequence (TCAGAGC, Figure 17D) corresponds to the consensus sequence of an initiator element (Inr) (69). Inr elements are commonly utilized as transcription start sites in TATA-less lymphoid-specific genes such as TdT (70). In agreement with this, we found no evidence of a canonical TATA box in the sequence of this region. Moreover, the relative position of the Inr corresponds to the major site of DNaseI hypersensitivity. In contrast to both mouse and human Gfil which generate multiple sized transcripts, the rat locus generates a single transcript (8). No start 
sites were detected in the first intron of the rat gene (data not shown). Thus, the Gfil rat locus appears to have a single start site corresponding to a consensus Inr sequence.

Mouse and human $G f i 1$ loci contain sequences homologous to the rat $G f i 1$ promoter including conserved GFI1 binding sites.

The sequence around the transcription start site of the rat Gfil locus was compared to the sequences of the mouse and human Gfil loci. This analysis revealed a great deal of homology among rat, mouse and human sequences up to 808 bp upstream of the putative Inr of the rat locus (Figure 18A, gray areas). This amount of sequence homology is unusual for non-coding DNA, emphasizing the probable importance of this region in the transcriptional control of Gfil.

The Inr sequence is conserved in the mouse locus, but not in the human sequences we identify here. However, the homologous region we show is defined as intron 1 of the human gene (71). Human Gfil is reported to have more than one transcription start site, and the major start site of the human Gfil promoter (72) contains a consensus Inr element by our analysis (data not shown).

Since Gfil is repressed in GFIl and GFI1B transgenic thymocytes, we next examined the sequence for GFI1/GFI1B binding sites by using a matrix similarity program (73). Putative GFI1/GFI1B binding sites that are conserved among all three species are annotated and underlined, as is the rodent $\operatorname{Inr}$ (Figure 18A). Furthermore, alignment of the first intron of the rat locus revealed extensive homology and two additional potential GFI1/GFI1B binding sites that are also conserved in all three species (data not shown).

GFI1 and GFI1B repression is conserved in the rat Gfil promoter. 
Figure 18. The rat Gfil promoter is highly homologous to mouse and human loci, and is sensitive to repression by GFI1 and GFI1B. A. Sequence alignment of mouse and human Gfil loci to the rat Gfil promoter. Regions in gray represent areas of identity among all three species. GFI1 binding sites and the Inr are underlined and annotated. Numbering is according to the rat sequence with ${ }^{+} 1$ as the first nucleotide in the putative Inr. B. The Gfil-promoter CAT reporter was cotransfected into 293T cells with CMV5 expression vector, or CMV5 expression plasmids encoding GFI1, GFI1B, or the repression-mutant P2A-GFI1. Transfection efficiency was controlled by cotransfection of a $\beta$-galactosidase expression construct. Relative CAT activity was derived by normalization to $\beta$-galactosidase levels within individual transfections. The plot represents the averaged normalized CAT activity \pm SEM from triplicate transfections. Similar results were obtained in three independent experiments. C. The VP16-Zn mutant encodes the transcriptional activation domain of VP16 fused to the DNA-binding Zn fingers of GFI1B. VP16-Zn activates transcription of GFI1/GFI1B target genes (10). The Gfil-promoter CAT reporter was cotransfected with CMV5 expression vector, or CMV5 expression plasmids encoding VP16-Zn. Normalization was performed as in A. Fold activation was derived by dividing the normalized CAT activity in the presence of VP16-Zn to CMV5 vector controls. 


\section{Figure 18}

A

$-808$

GFI1

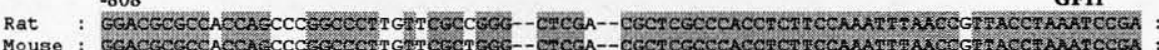

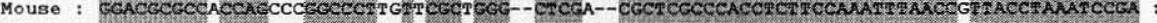

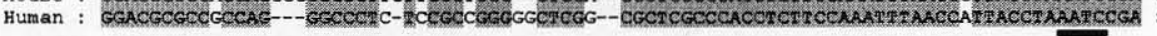

$-727$

GFI1

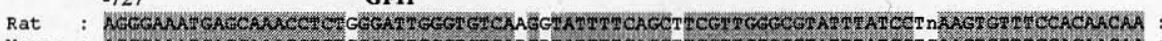

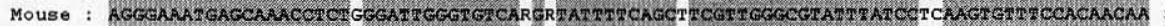

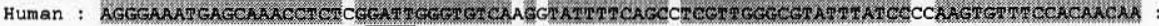

$-642$

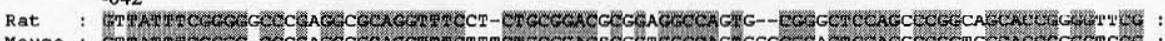
Human : GCHATH

$-560$

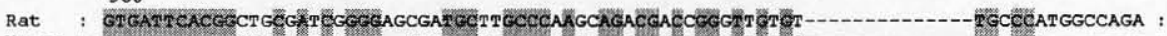

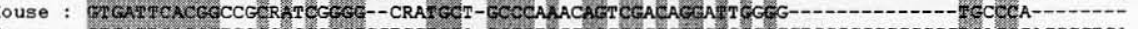

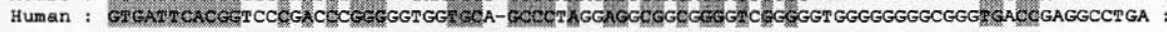
$-489$
GFI1
GFI1

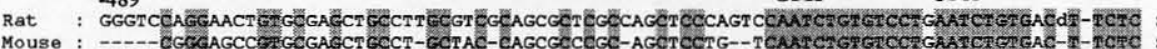

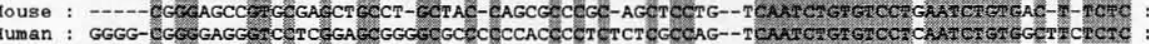

$-405$

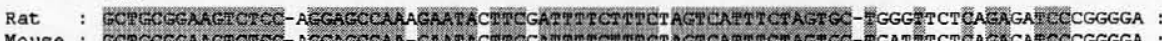

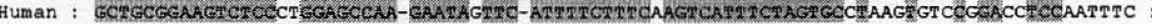

$-322$

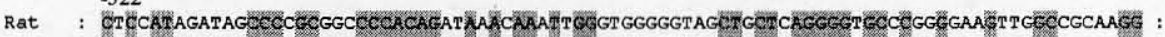

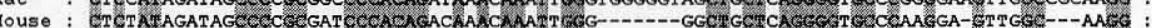

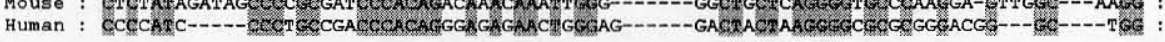

$-237$

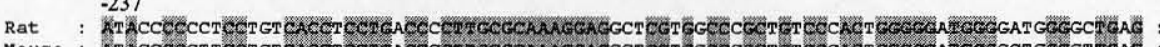

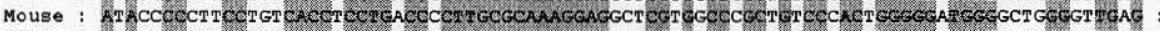

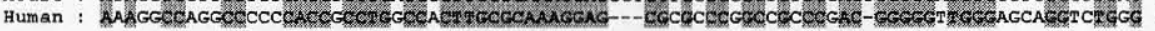

$-142$

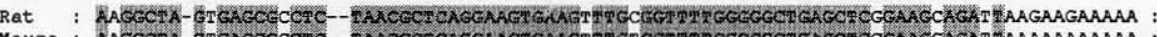

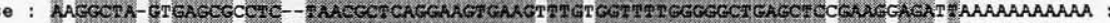

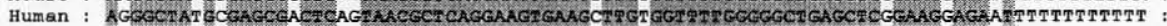

$-60$

GFII

INR

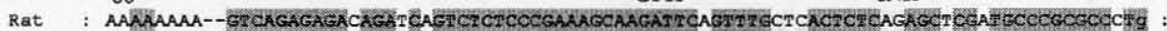
Mouse : AAAAAAAAAG

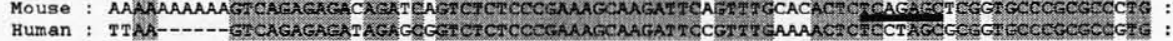

B
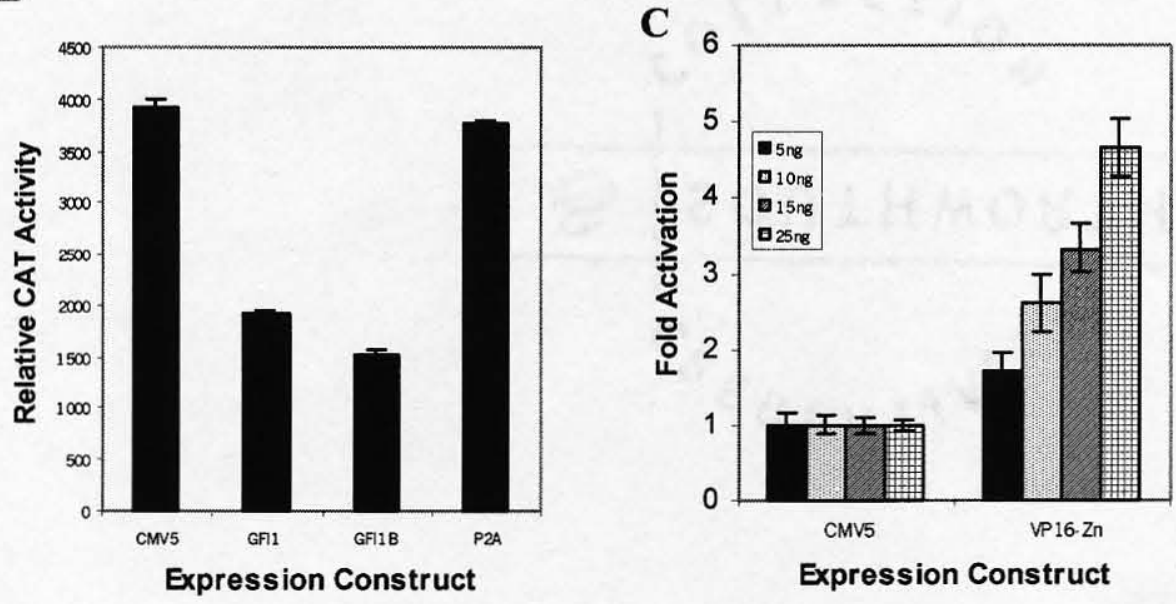

Expression Construct 
We next created a rat $G f i$-promoter-reporter construct containing the sequence from -808 bp 5 ' of the transcription start site to exon 2 . This sequence contains all of the DNase hypersensitive sites identified in the promoter region and first intron. Moreover, this region is highly conserved between mouse, rat and human Gfil loci. Transfection of several truncations of this construct into two different GFIl expressing cell lines resulted in significant increases in reporter activity upon removal of the region between -808 and 245 (data not shown). Therefore, a negative regulatory element is present in the GFI1 promoter.

293T cells are transformed kidney epithelial cells that express a low level of GFI1 (data not shown), and were used for transient transcription assays. As shown in Figure 18B, co-transfection of a Gfil promoter-driven CAT reporter construct and CMV5 expression constructs encoding GFI1 or GFI1B resulted in decreased reporter activity in comparison to empty CMV5 vector $(\mathrm{P}=0.006$, and $\mathrm{P}=0.002$ respectively). However, co-transfection of the repression-defective P2A-GFI1 has no effect on reporter activity (Figure 18B). While these data provide the expected result that GFI1 and GFI1B can repress the Gfil promoter and that the repression observed is mediated by the SNAG domain, they do not recapitulate the potent de-repression of the Gfil promoter as seen in P2A-GFI1 transgenic thymocytes. We consider that 293T cells may not express enough endogenous GFI1 to effectively repress multiple copies of the transfected reporter construct, and that transient transcription assays do not reproduce all aspects of chromatin-regulated gene expression.

To examine whether the repression of the rat Gfil promoter is due to direct binding of GFI1 to the promoter region, we utilized an expression construct encoding the 
activation domain of the herpes simplex virus VP16 protein fused to the zinc fingers of GFI1B (VP16-Zn) (10). This construct encodes a transcriptional activator with the DNAbinding specificity of GFI1/GFI1B. Co-transfection of the Gfil-CAT reporter with the VP16-Zn expression vector resulted in a dose-dependent increase in CAT activity (Figure 18C), suggesting that the observed repression by GFI1 and GFI1B is a result of direct binding of the promoter by GFI1 and GFI1B. The high frequency of conserved putative GFI1/GFI1B binding sites, along with the observed GFI1- and GFI1B-mediated repression of both endogenous Gfil in transgenic mice and rat Gfil promoter-reporter constructs, indicate that $G f i l$ is a direct target of GFI1 and GFI1B transcriptional repression in mice and rats.

\section{Human Gfil is repressed upon forced expression of GFI1 or GFI1B.}

To determine if human Gfil is also responsive to GFI1 and/or GFI1B, we transfected a human T cell line, Jurkat, with a selectable marker and the Lck-CD2-GFI1 and -GFI1B constructs and selected stable clones. Western blot analysis revealed varying expression levels of GFI1B (Figure 19A, clones 1B.1 and 1B.2), and high levels of GFI1 (Figure 19B, clones 1.1 and 1.2). Northern blot analysis of poly- $\mathrm{A}^{+}$selected RNA (Figure 19C) with a human Gfil-specific probe revealed that forced expression of GFI1 or GFI1B resulted in repression of endogenous human Gfil in Jurkat T cells.

Furthermore, the repression by GFI1B is dose-dependent in that the clone expressing higher levels of GFI1B has lower steady state levels of Gfil RNA (compare Figure 19A and Figure 19C, clone 1B.1 versus 1B.2). In contrast to the mild repression of Gfil in low-level-expressing GFI1 transgenic mice (Figure 16B), high levels of GFI1 result in a dramatic decrease in steady state levels of Gfil mRNA in Jurkat T cells (compare Figure 
Figure 19. GFI1 and GFI1B repress endogenous human Gfil in the Jurkat T-ALL cell line. A and B. Western blot analysis of GFI1B (A, 1B.1 and 1B.2) and GFI1 (B, 1.1 and 1.2) in independent stably transfected and selected Jurkat clones. Blots in A and B were probed with the same antisera as in Figure 16. C. Northern blot analysis of poly$\mathrm{A}^{+}$RNA (5 ug) from Jurkat clones using a probe specific for human Gfil. Ethidium bromide staining of the poly- $\mathrm{A}^{+} \mathrm{RNA}$ is shown for equal loading. Similar results were obtained with other clones. 
Figure 19

A.

B.
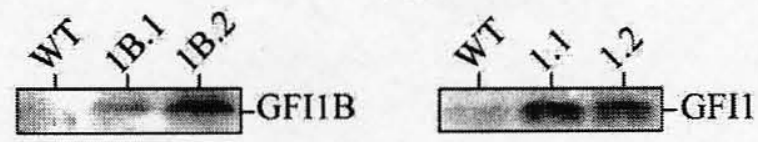

C.

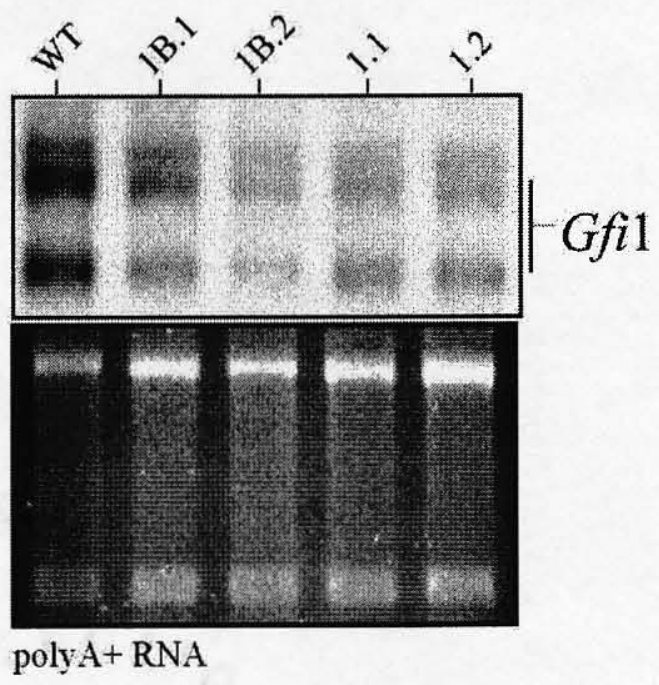


19B and Figure 19C, clones 1.1 and 1.2). Therefore the regulation of $G f i 1$ by GFI1 and GFI1B is conserved among mice, rats and humans.

\section{Jurkat nuclear extract can form in vitro protein/DNA complexes with putative GFI1 binding sites in the rat $G f i 1$ promoter and intron.}

The GFI1 consensus binding site has been defined by in vitro studies as TAAATCAC(A/T)GCA, with an absolute requirement for the AATC core $(10 ; 15)$. To determine which of the putative sites in the Gfil promoter bind to endogenous GFI1, we designed 30-bp oligonucleotide probes for use in electrophoretic mobility shift assay (EMSA) with Jurkat nuclear extracts. We observed several different protein/DNA complexes, some of which were unique to individual probes, and most of which were relatively minor in intensity. However, one of the probes (GRP2), corresponding to a putative GFI1 binding site at -705 (rat AATC core relative to Inr), was strongly shifted in a doublet with greatly retarded mobility (Figure 20). Another probe (GRP4), containing two putative binding sites located at -434 and -421 , shifted with the same pattern, but at a much lower intensity. In order to begin to discern whether any observed binding may suggest functionality, we engineered a probe to contain a GFI1 binding site that has been shown to be active in transient transcription assays (B30GRP; (9)). This probe contained the $\mathrm{B} 30$ binding site and flanking sequence from a putative binding site located at -558 in the rat promoter, and was named $\mathrm{B} 30 \mathrm{GRP}$. This probe shifted in the same doublet pattern as did GRP2 and GRP4; however, the intensity of the shifted band was significantly less than that observed for either endogenous promoter sequence. Complex formation was competed away by adding excess cold oligonucleotide (Figure 20). 
Figure 20. Formation of protein-DNA complexes with Jurkat nuclear extract and oligouncleotide probes containing GFI1 consensus binding sites in rat Gfil promoter sequence. A. Of seven oligonucleotide probes containing a total of eight predicted GFI1 recognition sites (GRP4 has two sites) in the rat Gfil proximal promoter, only GRP2 ($705)$ and GRP4 (-434 and -421) display appreciable binding to proteins in Jurkat nuclear extract. Migration of the complexes formed with these two probes is identical to the migration of complexes formed between Jurkat nuclear extract and a synthetic consensus GFI1 binding site (B30GRP). Note: These data were generated by Morgan Jeffries under the direction of Loretta Doan. B. A probe containing two putative GFI1 binding sites located in the intron of the rat and mouse genes (GRI) also forms these complexes when incubated with Jurkat nuclear extract. C. The sequence of the rat promoter with the locations of the probes indicated. Each probe sequence is underlined, and the AATC core of each putative GFI1 binding site is in bold. The italicized nucleotide is contained in both GRP1 and GRP2. The INR is also indicated in bold. 


\section{Figure 20}

A
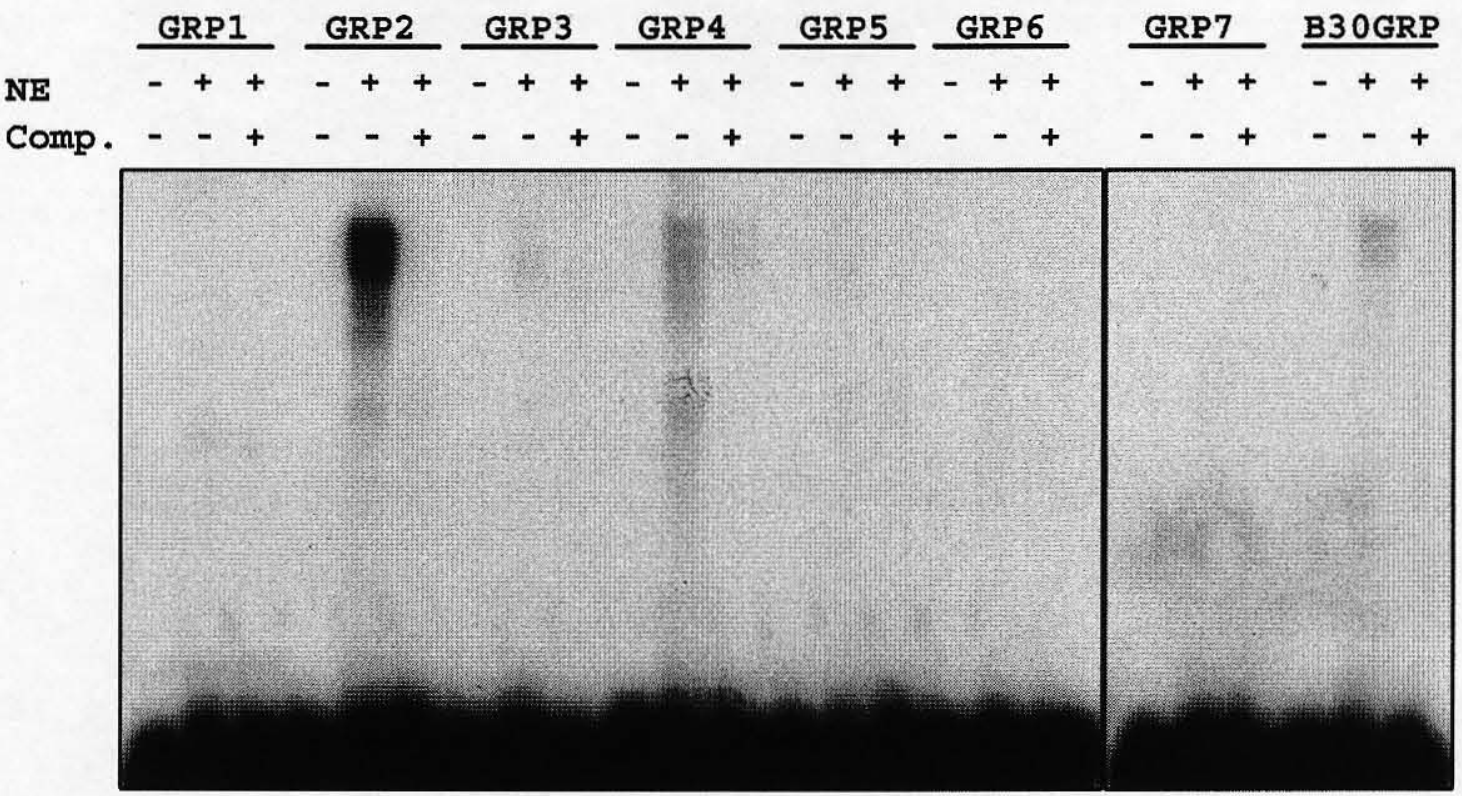

B

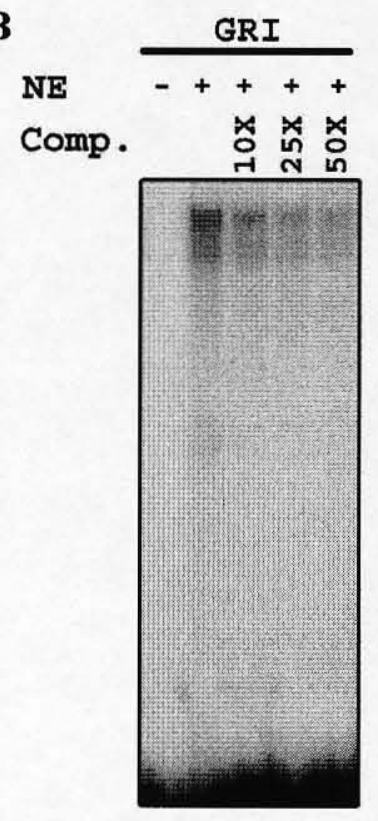

C

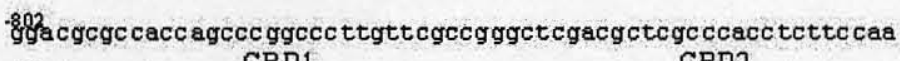
GRP1
Gat
GRP2 taacc gttacctaaatccgaagggaatgagcaaacctctggyattgggtgtcaag .684attttcagcttcgttgggcgtatttatcctcaag tgtttccacaacaagttatttcg

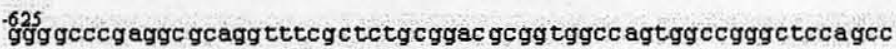
.566 GRP3 egcagc accggggttc ggtgatt cac gget gcgatcggggagc gatgcttgcccaagc -507 $a g g$ cgacc gggttgtgt tgcccatggc cagag ggtc caggaactgt gcgagctgcctgc.

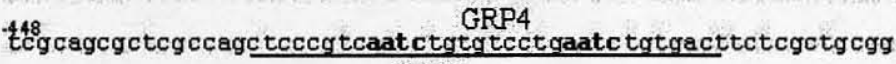
GRP5
-389g tctcc aggagccaagaatacttcgattttctttctagtcatttctagtgctgggtt ${ }^{330}$ ctc agaca tecc ggggactcca tagatagccc egcggeccc acaga taaac aaattggg -271 "212 .153 gatgggge tgagaagge tagtgagcgc ctctaacgc tcaggaagtgaagtttgcgg ttt $.94 \quad$ GRP6 tgggggct gagc tcggaagcagattaagaagaaaaaaaaaagtcagagagacaga tca GRPF
gtc tctcccgaaagcaagattcagtttgctcactct $\mathrm{NR}$
INagagctcgatgccegcgc cctg 
Further examination of potential regulatory elements in the rat intron revealed two putative GFI1 binding sites that are conserved in the mouse intron (data not shown). These sites lie close together and are both contained in the probe GRI. Figure 20 shows that this probe shifts in a doublet pattern identical to that observed with the promoterbased probes, and that this binding can be competed by the addition of excess cold probe.

\section{GFI1 binds directly to a predicted GFI1 binding site in the rat Gfil promoter.}

To determine whether GFI1 is present in, and required for, the complexes observed in the doublet, we more closely examined the properties of complexes formed between Jurkat nuclear extract and GRP2. Pre-incubation of Jurkat nuclear extract with antiserum against GFI1 disrupted the complexes (Figure 21A); no effect was noted using a control antiserum. Additionally, as observed in Figure 20, complex formation was efficiently competed in the presence of excess cold wild-type probe, but not in the presence of excess cold mutant probe (AATC->GGTC). Finally, when the mutant probe was labeled and incubated with Jurkat nuclear extract, the doublet was not detected. Combined, these EMSA results demonstrate that complex formation on the -705 oligonucleotide requires both GFI1 and the Gfil recognition sequence, strongly suggesting that GFI1 is binding directly to this site. Similar results were obtained with other potential binding sites for GFI1 (data not shown).

To ensure that GFI1 and GFI1B are capable of binding to the -705 site, we performed EMSA with in vitro transcribed and translated (IVT) proteins. As published by others (14), we note that IVT mouse GFI1B results in two proteins (Figure 21B). IVT GFI1 and GFI1B shifted the -705 oligonucleotide in EMSA (Figure 21C), and two protein-DNA complexes are seen in the presence of the two GFI1B products. GFI1 


\section{Figure 21. GFI1 and GFI1B bind in vitro to a conserved site in the rat Gfil}

promoter. A. EMSA analysis of GFI1 binding to the conserved site at $-705 \mathrm{bp}$. Jurkat nuclear extract incubated with the -705 bp probe alone (lane 2) forms several distinct protein-DNA complexes. Supershift analysis was performed with a goat polyclonal antibody to GFI1 (lane 3). Pre-immune goat sera served as control (lane 4). Cold competition was performed with a 30-fold excess of cold probe (lane 5), or cold mutant probe (AATC to GGTC) (lane 6). Incubation of Jurkat nuclear extract with a labeled mutant probe (lane 6). $\mathrm{W}=$ wild type probe, $\mathrm{M}=$ mutant probe, $\mathrm{G}=$ goat anti-GFI1 antiserum, $\mathrm{Cg}=$ control goat antiserum. B. In vitro transcribed and translated GFI1, GFI1B, or a mutant of GFI1 lacking the fourth zinc finger $(\Delta \mathrm{Zn} 4)$ were ${ }^{35} \mathrm{~S}$ labeled, run on a denaturing polyacrylamide gel, and subjected to autoradiography. Representative autoradiographs are shown. C. IVT GFI1 and GFI1B, but not $\Delta$ Zn4 GFI1, form complexes with the -705 oligonucleotide probe. Note: These data were generated by Morgan Jeffries under the direction of Loretta Doan. 
Figure 21

A

$\mathrm{NE}:-++++++$ Probe: W W W W W W $M$ Ab: - : $\mathrm{G} \mathrm{Cg}-\overline{\mathrm{W}} \dot{\mathrm{M}}$ :

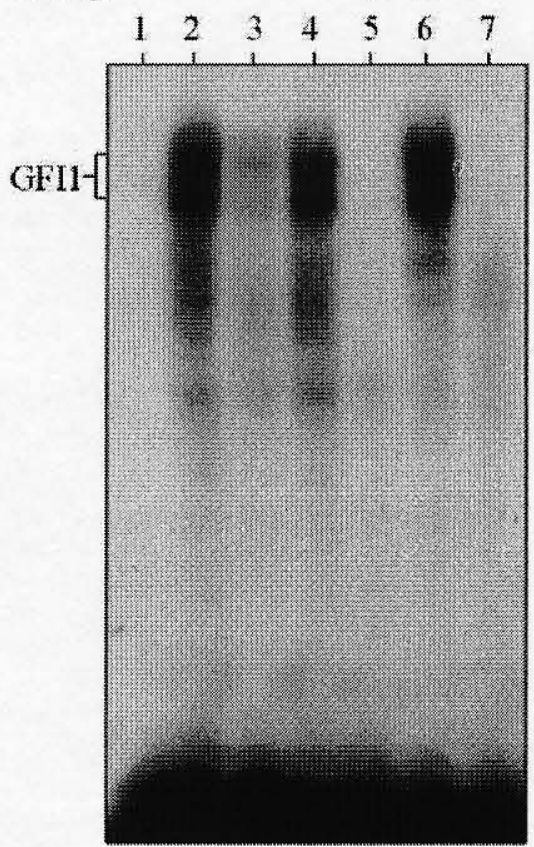

B

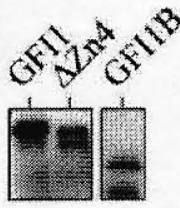

C.

IVT: - $\mathrm{O}^{2} \mathrm{y}^{\mathrm{x}}$

Probe: $W$ W W W

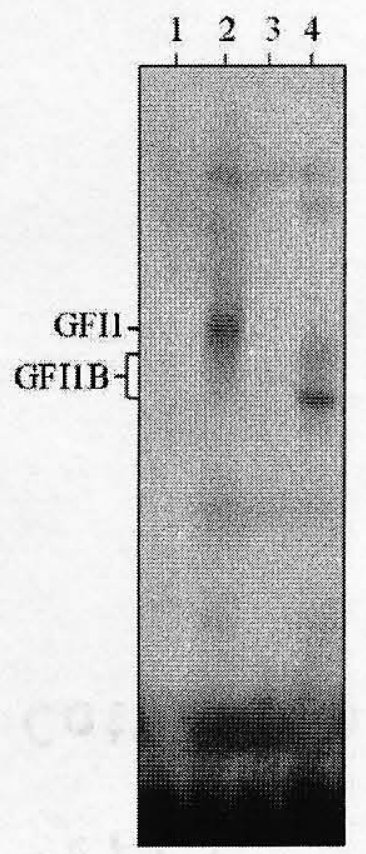


requires zinc fingers 3,4 , and 5 for sequence-specific DNA binding (15). As expected, EMSA using a mutant of GFI1 lacking zinc finger $4(\Delta \mathrm{Zn} 4$; Figure $21 \mathrm{C})$ did not result in retarded mobility of GRP2. These data support the suggestion that GFI1 in Jurkat nuclear extract is binding directly to these identified sites. Furthermore, the relatively faster mobility of the complex formed between DNA and IVT GFI1, as compared to the mobilities of the two bands in the doublet observed with Jurkat nuclear extract, suggests that in nuclear extract, GFI1 exists in complexes with other proteins.

\section{Potential differences in DNA sequence requirements for binding by GFI1 in nuclear complexes.}

The GFI1 consensus site was defined by using synthetic oligonucleotides and bacterially synthesized proteins encoding GST fused to GFI1 (15). This sequence is utilized by the matrix homology program MatInspector (73) to identify putative GFI1 binding sites in the promoters of user-defined genes. When MatInspector identifies putative recognition sequences, it also assigns a percent consensus score to the sites in question. We limited our analyses to sites that had a $90 \%$ or greater score. We expected those sites with the highest scores to show the most efficient binding to GFI1. However, the relative binding differences we observed in our EMSA analysis using Jurkat nuclear extract as a source for GFIl did not agree with the predicted affinities. Due to the limited number of oligonucleotide probes (eight), our initial examination failed to identify a common sequence requirement among those that bound GFI1 when compared to those that did not. However, upon further examination of the promoter sequence, and comparison to the public domain sequences that became available during the course of this work, we discovered seemingly minor discrepancies in the sequences of both GRP2 
and GRP4. (The other sequences used in probes of putative binding sites were verified as accurate by the same analyses.) Specifically, the apparently correct sequence of GRP2 has an A at the position +6 from the first A of the AATC core (instead of the T in our original probe), while GRP4 contains a $\mathrm{T}$ at position -2 (instead of the $\mathrm{C}$ in our original probe) (Figure 22A). We predicted that neither change in sequence would alter binding by GFI1 because, in the in vitro experiments that first defined the GFI1 consensus sequence, both nucleotides were selected in approximately the same number of oligonucleotides in each case (15). To test this prediction, we re-synthesized the oligonucleotide probes with the correct sequence and performed EMSA to compare GFI1 binding.

As seen in Figure 22B, the $T$ to A change in the sequence of GRP2 affected neither the pattern nor the intensity of complex formation (compare GRP2 and GRP2.2). However, the $\mathrm{C}$ to $\mathrm{T}$ alteration of the first site in GRP4 drastically changed the pattern of complex formation (compare GRP4 and GRP4.2). GRP4.2 shifts in a single complex with a somewhat faster mobility than the lower band in the doublet observed with GRP2, GRP2.2, and GRP4. Competition assays defined this first site as being solely responsible for the observed GFI1 binding in both GRP4 probes (data not shown). This suggests that there are several GFI1-containing complexes in Jurkat nuclear extract, and that changing the nucleotide at -2 from $\mathrm{C}$ to $\mathrm{T}$ alters the ability of these complexes to bind. Similar results were obtained when nuclear extract from $\mathrm{Nb} 2$ cells were used as the source of GFI1 (data not shown). While suggestive, these data are certainly not conclusive, and this hypothesis remains to be tested. 


\section{Figure 22. Jurkat $T$ cell nuclear extracts contain multiple GFI1 complexes with}

specific DNA sequence requirements and varying DNA binding affinities. $A$.

Sequence alignment of oligonucleotide probes. The sequences of the original probes are indicated as GRP2, GRP4, GRI, and B30GRP. Probe GRP2 contains the site at -705 , and probe GRP4 contains two binding sites at positions -434 and -421 . Probe B30GRP is a consensus GFI1 binding site with flanking sequence from the Gfil rat promoter that surrounds a putative binding site at -558 . The ultimately resolved sequences of the rat promoter are indicated as GRP2.2 and GRP4.2. The changed nucleotides are shown in bold. B. GRP2, GRP2.2, and GRP4 show the same binding pattern, but GRP4.2 forms a single complex with greater mobility than either of those formed with the other three probes. C. Cross-competition confirms the relative binding affinities of oligonucleotide probes (Figure 20). GRP2.2 was radiolabeled and used as probe (lane 1, probe only; lane 2, probe with Jurkat nuclear extract). Competition analysis was performed using increasing molar amounts (10X, 25X, and 50X the ng quantity of probe) of GRP2.2 (lanes 3-5), GRP4.2 (lanes 6-8), GRI (lanes 9-11), mut1GRI (the first AATC mutated to GGTC; lanes 12-14), mut2GRI (the second AATC mutated to GGTC; lanes 15-17), and mut3GRI (both AATC mutated to GGTC; lanes 18-20). GRP2.2, GRI, and mut2GRI all compete with GRP2.2 for binding to GFI1 in Jurkat nuclear extract. GRP4.2, mut1GRI, and mut3GRI do not compete. (NE = Jurkat nuclear extract.) 
Figure 22

A

$\begin{array}{ll}\text { GRP2 } & \text { CCAATCCCTGAG } \\ \text { GRP2.2 } & \text { CCAATCCCAGAG } \\ \text { GRP4 } & \text { CCAATCTGTGTC } \\ \text { GRP4.2 } & \text { TGAATCTGTGAC } \\ \text { TCAATCTGTGTC } \\ \text { GRI } & \text { TGAATCTGTGAC } \\ \text { BCAATCAGTTCA } \\ \text { B0GRP } & \text { CTAATCTCGGGT } \\ & \text { CAAATCACTGCA }\end{array}$

B
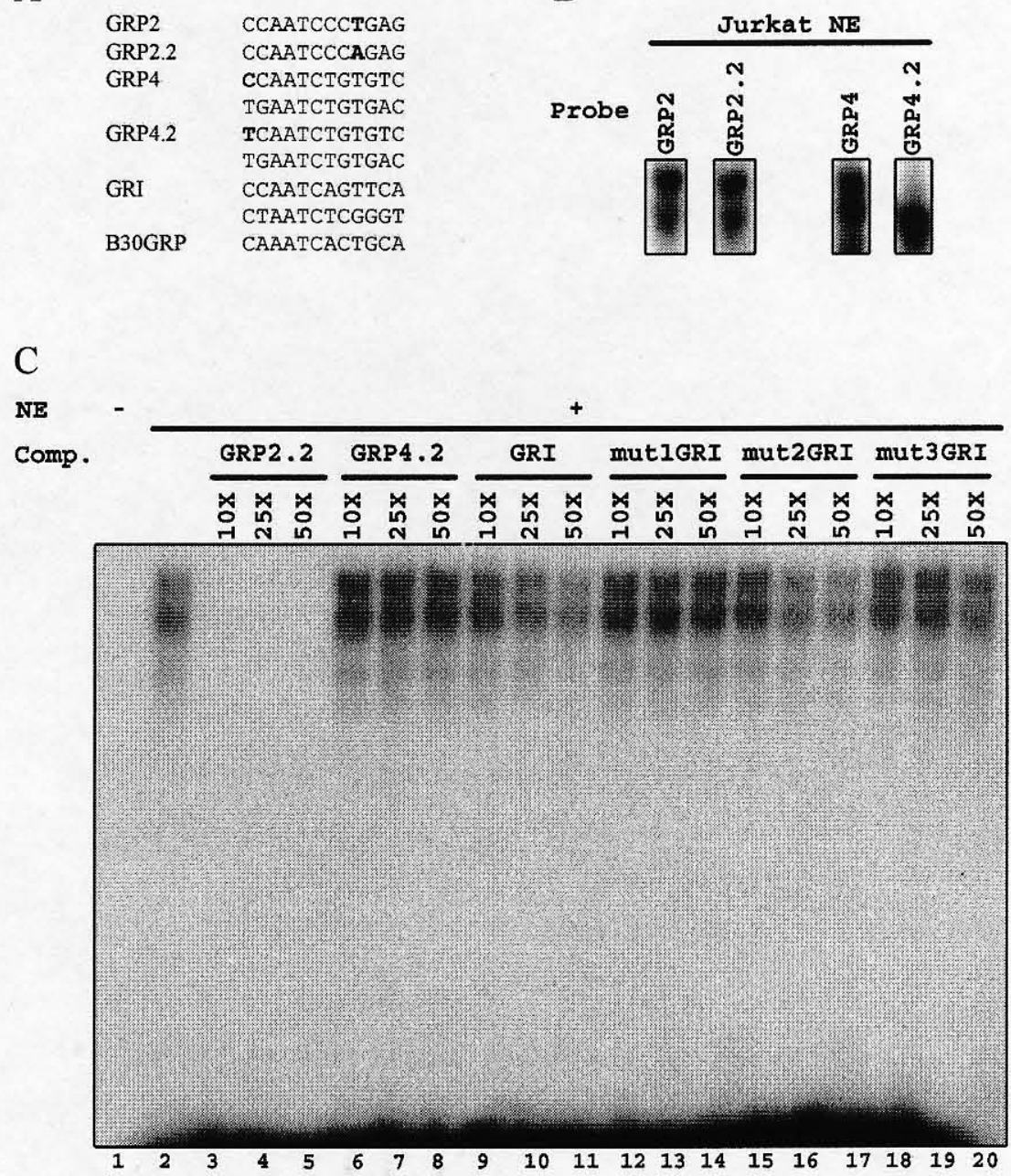
To ensure that the observed differences in intensities of the complexes are a result of the relative strength of binding by GFIl and not due to artifact such as poor probe labeling, we performed cross-competition analysis (Figure 22C). In this assay, GRP2.2 was used as radiolabeled probe, and competition was performed using different unlabeled oligonucleotides. This analysis showed that the corrected site at -421 (GRP4.2) does not compete away the binding to the site at -705 (GRP2.2). Furthermore, it confirmed that the affinity for GFI1 binding to GRP2 is greater than that for binding to the intronic sites (GRI), as GRI does not effectively compete for binding unless added in 25 or 50 fold excess, whereas GRP2. 2 completely competes its own binding at 10 fold excess. The reverse experiment was also done, in which GRP2.2 effectively competes all binding by GRI when added in 10 fold excess (data not shown). In addition, analysis of the AATC to GGTC mutants of each of the putative binding sites in GRI reveals that the first site is solely responsible for competition; when this site is mutated, GRI is unable to compete with GRP2 even at 50 fold excess (compare lanes 9-11 and 12-14). However, mutation of the other intronic site did not affect competition (compare lanes 9-11 and 15-17). Therefore, the first of the two predicted GFI1 binding sites in the intron is bound by GFI1, whereas the second is not. Furthermore, the site at -705 binds more GFI1 complex than does any other GFI1 binding site identified in our in silico analyses.

\section{GFI1 autoregulation is not observed in a myeloid lineage cell line.}

Both $G f i 1^{-/-}$mice and $G f i l$-mutant humans display profound neutropenia $(12 ; 16 ; 17)$, indicating a role for GFI1 in myeloid differentiation. U937 is a bi-potential human myeloid-precursor cell line that can differentiate into monocytes or neutrophils (74). U937 cells express GFI1 (13). To determine if Gfil autoregulation is active in non- 


\section{Figure 23. GFI1 autoregulation occurs in $T$ cells but not in a myeloid-lineage cell}

line. A. Western blot analysis of human U937 cells transduced with retroviruses encoding Flag-tagged GFI1 with an anti-Flag antibody (top) or anti-GFI1 antibody (bottom). Note: These data were generated by Dr. Christopher M. Jay. B. Northern blot analysis of endogenous Gfil transcripts using a probe specific to the human Gfil 3' untranslated region. C - F. Chromatin immunoprecipitation analysis of wild type Jurkat T cells or U937 cells using either rabbit or goat polyclonal antisera to GFI1 ( $\alpha$ GFI1 Ab). Non-specific rabbit and goat sera served as control (control Ab). Primers for the amplification reactions targeted the promoter or $3^{\prime}$ untranslated region of human Gfil. Input $=0.005$ to $0.01 \%$ of input chromatin. $\mathrm{J}=$ Jurkat. $\mathrm{U}=\mathrm{U937.} \mathrm{P}=$ promoter. $3^{\prime}=3^{\prime}$ region of $G f i 1 . \mathrm{R}=$ rabbit polyclonal antiserum $\mathrm{G}=$ goat polyclonal antiserum. Note: These data were generated by Zijun Duan and Marshall Horwitz (Department of Medicine, Division of Medical Genetics, University of Washington School of Medicine). 
Figure 23
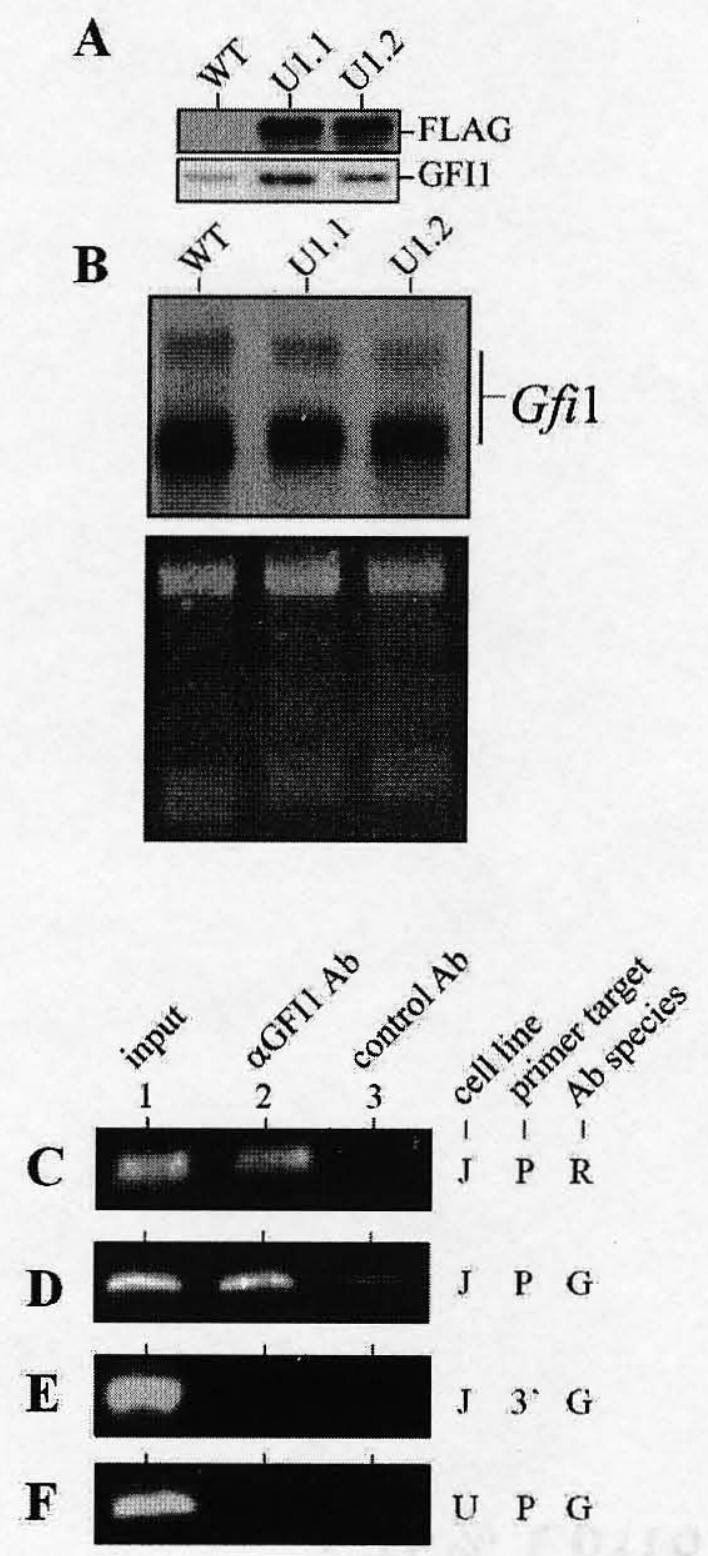
lymphoid lineages we transduced U937 cells with retroviral vectors encoding Flagepitope-tagged GFI1 (Figure 23A, U1.1 and U1.2). This Flag-tagged GFI1 was confirmed to be an active transcriptional repressor in transient transcription assays (data not shown). The transduced U937 cells express the epitope-tagged GFI1 and have higher levels of GFI1 overall (Figure 23A); in fact, the level of expression was similar to that observed in the stably transfected Jurkat T cells (compare Figure 19B with Figure 23A). However, we did not observe profound repression of the endogenous Gfil locus in U937 cells (Figure 23B). While there appears to be a mild reduction in the expression of endogenous $G f i 1$, it does not compare with the repression observed in Jurkat T cells. We therefore conclude that, unlike T lymphocytes, U937 myeloid cells do not display effective $G f i l$ autoregulation.

\section{GFI1 binds the Gfil promoter in live $T$ cells.}

To determine the biological relevance of $G f i l$ autoregulation, we performed chromatin immunoprecipitation (ChIP) analysis of cross-linked protein-DNA complexes from Jurkat $\mathrm{T}$ cells. This technique reveals protein-DNA interactions within the context of living cells. Amplification using primers that flank the -705 GFI1 binding site resulted in a PCR product from chromatin immunoprecipitated with rabbit antisera to GFI1, but not when immunoprecipitation was performed using control antisera (Figure 23C). Essentially the same result was found with goat antisera to GFI1 and control goat antisera (Figure 23D). In contrast, using the same analysis, we were unable to amplify a product using primers to a 3' region of Gfil (that does not contain GFIl binding sites by our analyses) (Figure 23E). Thus, the ChIP result is specific to the promoter region of Gfil. While the ChIP analysis does not prove that GFI1 is specifically bound to the -705 site, 
these in vivo data show that GFI1 does bind its own promoter in living T cells.

Moreover, these data suggest that the Gfil autoregulation we observe is indeed a physiologically relevant cellular mechanism.

\section{GFI1 does not bind the Gfi1 promoter in living myeloid cells.}

We repeated ChIP analysis with the U937 cell line. In contrast to our results with Jurkat cells, we did not find GFI1 bound to the Gfil promoter in U937 cells (Figure 23F). These data provide a potential reason for the lack of repression observed in Figure 23B. GFI1 in U937 cells is capable of DNA binding, as we have published the binding of GFI1 to a number of promoters in this cell line (13). Furthermore, the general chromatin environment of the Gfil locus is permissive for DNA binding, as U937 cells express endogenous GFI1 (Figure 23A). It is of course possible that localized chromatin control may alter the promoter to make the GFI1 binding sites inaccessible. In any case, the ChIP analyses indicate that GFI1 autoregulation is not active in all cells that express Gfil, and may not occur in cells of myeloid lineage.

\section{Discussion}

GFI1 is a transcriptional repressor protein that plays important biological roles in hematopoietic and neuronal cell development $(11 ; 16 ; 17 ; 75)$. Gfil was originally identified in an in vitro screen for genes that, upon deregulated expression, engender progression of $\mathrm{T}$ cell leukemias to Interleukin-2 independent growth (8). Transgenic overexpression of GFI1 is poorly oncogenic, but potently collaborates with transgenic expression of MYC or PIM oncoproteins to cause leukemia (76). In contrast, deletion of $G f i l$ leads to profound lymphopenia (16;17). Since Gfil expression is critical for normal 
hematopoiesis, and overcoming regulatory control of $G f i l$ accelerates oncogenesis, the transcriptional control of Gfil is of great interest. Here we have shown that GFI1, and the closely related GFI1B, both repress the transcription of mouse, rat, and human Gfil.

We present several lines of evidence supporting a direct and physiologically relevant mechanism of Gfil repression by GFI1. First, GFI1 binding sites in the rat Gfil promoter are conserved in the mouse and human Gfil loci. Second, in primary thymocytes, transgenic expression of GFI1 correlates with repression of endogenous Gfil steady-state mRNA levels, whereas expression of a repression-defective GFI1 mutant correlates with a profound de-repression of murine Gfil. Third, in the human Jurkat $\mathrm{T}$ cell line, forced overexpression of GFI1 correlates with reduced endogenous Gfil steady-state mRNA levels. Fourth, in EMSA analysis, in vitro transcribed/translated GFI1 and endogenous GFI1-containing nuclear complexes bind sequences in the rat Gfil promoter. Finally, ChIP analyses with two different antisera indicate that GFI1 binds to its own promoter in living Jurkat $\mathrm{T}$ cells. These in vitro and in vivo analyses provide strong support for the hypothesis that GFI1 regulation of $G f i 1$ is physiologically relevant. In fact, Gfil autoregulation is evolutionarily conserved, as pag-3, the Caenorhabditis elegans ortholog of Gfil, is autoregulated (77).

GFI1 and GFI1B control Gfil expression. The abilities of GFI1 and GFI1B to repress endogenous Gfil seem comparable. Similar expression levels of the two factors in stably transfected Jurkat $\mathrm{T}$ cells resulted in similar repression. Furthermore, cotransfection of equal amounts of GFI1 and GFI1B expression plasmids with a Gfil promoter-driven reporter resulted in nearly equal levels of repression. The regulation of Gfil by GFI1B is reciprocated. In addition to the data presented here, ChIP analysis in 
KG-1, U937, and Jurkat cells using antibodies against GFI1 demonstrated that GFI1 binds to the GfilB promoter region in living cells (13). Together, these data suggest cross-regulation of Gfil and GfilB.

GFI1/GFI1B cross-regulation provides a potential explanation for differential steady-state expression of $G f i 1$ and $G f i 1 B$, and suggests a molecular mechanism to ensure that the function of the properly expressed transcription factor is not blocked by aberrant expression of the other. We note that both factors can be simultaneously expressed (19). Specifically, Gfil and $G f i l B$ are co-expressed during specific stages of T cell development coincident with activation (19). The repression of Gfil by GFI1B provides new insight into the transient expression of GFI1B in thymocytes that have been recently signaled for positive selection. Namely, GFI1B may be present to temporarily repress Gfil, thereby decreasing the potentiative effect of GFI1 on TCR stimulation and finetuning the cellular response to activation. Since GFI1 increases T cell activation (19-21), GFI1B expression may indirectly inhibit T cell activation through the repression of Gfil.

Mutation of GFI1 in both mice and humans causes profound neutropenia $(12 ; 16 ; 17)$. Since the primary function of neutrophils is to defend against bacteria and fungal infection (78), humans with either Severe Congenital Neutropenia (SCN) or "nonimmune chronic idiopathic neutropenia of adults" (NI-CINA) are predisposed to opportunistic infections (79). We have recently shown that GFI1 is mutated in SCN and NI-CINA patients without mutations in neutrophil elastase (ELA2) (12), the most prevalent mutation in SCN (79). In contrast to ELA2-mutant SCN patients, GFI1-mutant SCN patients are also mildly lymphopenic and have T cell activation defects (12). In mice, deletion of $G f i l$ results in a profound neutropenia that is strikingly similar to that 
seen in human GFI1-mutant SCN patients (16). However, the lymphoid phenotype of $G f i 1^{-/ 2}$ mice is dramatically worse than that of GFI1-mutant SCN humans $(16 ; 17 ; 17)$.

Cell type specific $G f i 1$ autoregulation may explain the disparity between the lymphoid phenotype of $G f i 1^{-/}$mice and Gfil-mutant humans. Our T-cell specific P2AGFI1 transgenic mice lack a Gfil-deleted phenotype and demonstrate significant derepression of the endogenous $G f i 1$ locus. Thus, by interfering with the transcriptional repression activity of endogenous GFI1, the P2A-GFI1 mutant may induce compensation in $\mathrm{T}$ cells to prevent a complete loss of GFI1 function. In fact, humans with SCN are heterozygous for mutant Gfil (12). In a manner similar to the P2A-GFI1 transgenic mice, the expression of the mutant GFI1 in SCN patient T cells might lead to derepression of the wild-type allele, providing compensatory expression of wild-type GFI1 and sufficient GFI1 activity in these cells. In contrast, myeloid progenitor cells, like the U937 myeloid lineage cell line, may not exhibit Gfil autoregulation. These cells would not have compensation by the wild-type allele and would effectively lose most or all GFI1 function. Thus, cell-type specific $G f i 1$ autoregulation provides a potential explanation for the differences in phenotype observed in $\mathrm{ffil}^{-/}$mice when compared to humans with a mutation in a single allele of $G f i 1$. 


\section{CHAPTER V}

\section{GFI1B INTERFERES WITH POSITIVE SELECTION OF TCR TRANSGENIC THYMOCYTES}

\section{Introduction.}

A central tenet of $\mathrm{T}$ cell-mediated immunological functions is the requirement for recognition of haplotype-specific MHC molecules by the TCR. Indeed, it is the combination of antigen and the haplotype of the presenting MHC molecule that determines the response or nonresponse of a mature $\mathrm{T}$ cell. The induction of an immune response requires that the TCR recognize foreign antigen in the context of self-MHC. On the other hand, the presentation of self antigen by self-MHC results in low-level TCR signaling and survival, but not activation, of $\mathrm{T}$ cells. This principle also governs the positive selection of thymocytes. Thymic antigen presenting cells present a wide variety of self antigen in the context of self-haplotype specific MHC molecules. In fact, it is thought that thymic APC have access to virtually all potential self-antigens, thereby providing to developing $\mathrm{T}$ cells the potential to interact with these self antigens. These interactions ensure both the development of a very diverse $\mathrm{T}$ cell repertoire and the clonal deletion of all potentially self-reactive $\mathrm{T}$ cells (recently reviewed in (80)). Furthermore, under normal physiological circumstances, thymocytes are restricted and selected by self$\mathrm{MHC}$ only, ensuring the recognition of foreign MHC as foreign. Therefore, the MHC 
haplotype that can successfully select a T cell bearing a specific TCR is fixed and can be experimentally determined.

This central principle has been exploited by researchers to generate tools that further enhance our understanding of TCR-MHC interactions. TCR transgenic mice have been generated in which $\mathrm{T}$ cells predominantly express only the engineered transgenic TCR. Both the MHC specificity and the antigenic specificity of these transgenic TCRs are known, allowing researchers to more closely examine TCR-antigen-MHC interaction as well as specific processes of $\mathrm{T}$ lymphopoiesis that are governed by these interactions, such as positive and negative selection. We have used these systems to further investigate the phenotype of our GFI1B transgenic mice.

Our initial phenotypic analysis of GFI1B transgenic thymi presented results that were unexpected in the context of accepted theories of thymocyte selection and maturation. Specifically, we found that GFI1B transgenic thymocytes and T cells are defective in TCR signaling (Chapter 2). Currently accepted theory of T lymphopoiesis suggests that a stronger or more persistent TCR signal is required for the formation of CD4 SP cells and that a weaker or less persistent signal results in the formation of CD8 SP cells (recently reviewed in $(81 ; 82)$ ). According to this theory, GFI1B transgenic mice should generate more CD8s and fewer CD4s when compared to wild type mice. Contrary to this, we discovered that GFI1B transgenic mice have an unusually high percentage and number of CD4 SP cells (Figure 10, chapter 2). Furthermore, these CD4 SP cells are phenotypically mature as determined by low expression of the maturation marker CD24/HSA. Given the down-modulation of TCR signals observed in GFI1Btransgenic $\mathrm{T}$ cells, we reasoned that the abnormal CD4 SP cells in GFI1B transgenic mice 
may be the result of aberrant positive selection of DP thymocytes that should have received a stronger signal and, therefore, should have been deleted.

Preliminary studies examining negative selection indicated little or no effect of the GFI1B transgene. These studies include flow cytometric apoptosis assays of thymocytes, examination of deletion of specific $\mathrm{V} \beta$ subsets in thymocyte populations, and expansion and survival of $\mathrm{V} \beta$ subsets in the periphery (data not shown). Because no difference was observed between GFI1B transgenic mice and wild type littermate controls in these experiments, we did not further investigate negative selection as a mechanistic explanation for the GFI1B transgenic phenotype. Rather, we decided to pursue positive selection as the mechanism.

To determine whether GFI1B affects positive selection, we crossed GFI1B transgenic mice with mice that express a transgenic TCR. All mice were crossed onto a $\operatorname{Rag} 2^{-/}$background so that no rearrangement of endogenous TCR loci would occur. In addition, to reduce the possibility of TCR transgene-specific effects, we used two different TCR transgenic lines, AND and DO.11. Both TCR transgenes are MHC Class II restricted and, when on a genetic background expressing the proper selecting MHC molecules, result in the positive selection of large numbers of CD4 SP cells (83-86).

We discovered that in these systems, transgenic expression of GFI1B results in a reduction in the number of CD4 SP cells, with a concomitant increase in the number of DP thymocytes. Furthermore, a large percentage of those CD4 SP cells that are generated are phenotypically abnormal, displaying properties of both mature and immature SP thymocytes. In addition, microarray analysis provides insight into changes in the transcription levels of genes that are known to contribute to $\mathrm{T}$ cell activation, and 
EMSA analysis provides preliminary evidence that these TCR signaling pathways are affected in GFI1B transgenic thymocytes.

\section{Materials and methods.}

Mice. $\mathrm{Rag}^{-/-}$TCR transgenic mice were obtained from Taconic Farms. These mice have bred true for generations and therefore transmit one copy of the transgene to all progeny. $\mathrm{Rag}^{-/-}$GFI1B transgenic mice were generated by crossing $\mathrm{Rag}^{-/-}$mice (Taconic Farms) with GFI1B transgenics, all on a B6 background. GFI1B transgenic, $\mathrm{Rag}^{+/-}$progeny were backcrossed to $\mathrm{Rag}^{-/-}$mice to generate $\mathrm{Rag}^{-/-}$GFI1B transgenic mice. These were mated with $\mathrm{Rag}^{-/-} \mathrm{TCR}$ transgenics to generate litters of $\mathrm{Rag}^{-/-} \mathrm{TCR}_{\text {transgene }}^{+}$and GFI1B ${ }^{+}$or GFI1B $^{-}$animals for experiments.

Flow cytometry and antibodies. Flow cytometric methods have been described in Chapter 3. Antibodies used include CD4 (RM4-5 and GK1.5) and CD8 $\propto$ (53-6.7), CD24 $\alpha$-(M1/69), CD69 (H1.2F3), and CD62L (MEL-14), all from BD Pharmingen. RNA isolation and microarray. For microarray analysis, single cell suspensions of thymocytes were prepared. Phenotypes of mice were confirmed by flow cytometric analysis, and RNA was extracted from thymocytes with UltraSpec RNA Isolation System (Biotecx) following the manufacturer's protocol. Microarray analysis was performed in the Brown Cancer Center Microarray core facility of the University of Louisville.

EMSA. Electrophoretic mobility shift assays were performed according to the protocol described in chapter 2 with the following modifications. Probes were oligonucleotides from Santa Cruz Biotechnology (sc-2501, sc-2505, and sc-2525) and were labeled with Polynucleotide Kinase (New England Biolabs) following a standard protocol. $2.5 \mu \mathrm{g}$ of 
nuclear extract was incubated with $1 \mathrm{uL}$ of radiolabeled oligonucleotide probe $(100,000$ $\mathrm{cpm}$ ). Complexes were electrophoresed through $6 \%$ polyacrylamide gels, dried, and exposed to film.

\section{Results and discussion.}

Transgenic expression of GFI1B results in decreased positive selection and the presence of abnormal MHC Class II restricted TCR transgenic thymocytes.

To determine the effect of transgenic expression of GFI1B on positive selection of thymocytes, we crossed AND transgenic mice to GFI1B transgenic mice. The resulting F1 progeny are $\mathrm{H}-2^{\mathrm{b} / \mathrm{d}}$, which is a positively selecting background for AND TCR transgenic thymocytes (83). When we examined the thymi of these progeny, we observed a large percentage of thymocytes were CD4 SP in the GFI1B- AND transgenic mice, in accordance with previously published results. However, when transgenic GFI1B is expressed, the percentage and number of CD4 SP thymocytes is decreased to approximately half those of controls (Figure 24A). The total number of thymocytes is not different between GFI1B transgenics and GFI1B- littermate controls, and the difference in the number of CD4 SP cells is inversely correlated with a difference in the number of DP cells. Similar results were obtained with DO.11/GFI1B bi-transgenics (Figure24B), though both the decrease in CD4 SP cells and the increase in DP cells in GFI1B transgenics are even more pronounced in this case. DO.11 mice do not produce the same large percentage of CD4 SP cells observed in AND transgenics (86), and our F1 
Figure 24. Transgenic GFI1B decreases the number of CD4 SP cells in Class II restricted TCR transgenic mice. A. Flow cytometric plot of CD4 v CD8 of Rag AND TCR transgenic and $\mathrm{Rag}^{-/}$AND TCR GFI1B bi-transgenic mice. DP and CD4 SP populations are gated, and percentage of total cells indicated. The MHC haplotypes of these F1 mice are $\mathrm{b} / \mathrm{d}$. The table below the graphs gives absolute numbers of cell populations. B. The same analyses were applied to $\mathrm{Rag}^{-/-} \mathrm{DO} .11 \mathrm{TCR}$ transgenic mice $\left(\mathrm{MHC}^{\mathrm{b} / \mathrm{d}}\right)$. 
Figure 24

A

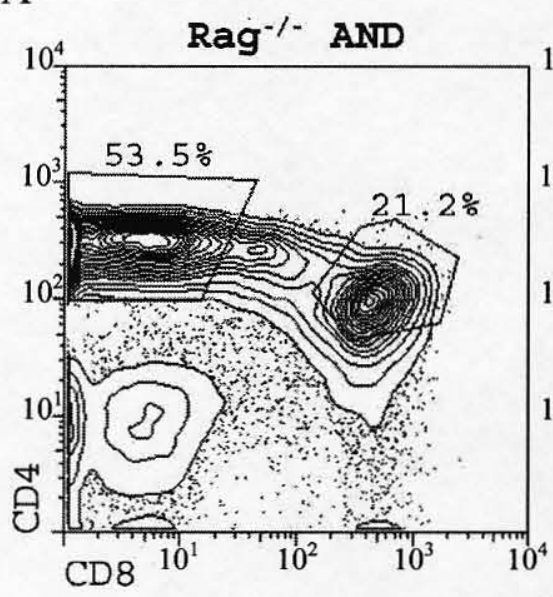

$\mathrm{Rag}^{-1 /}$ AND GFIIB

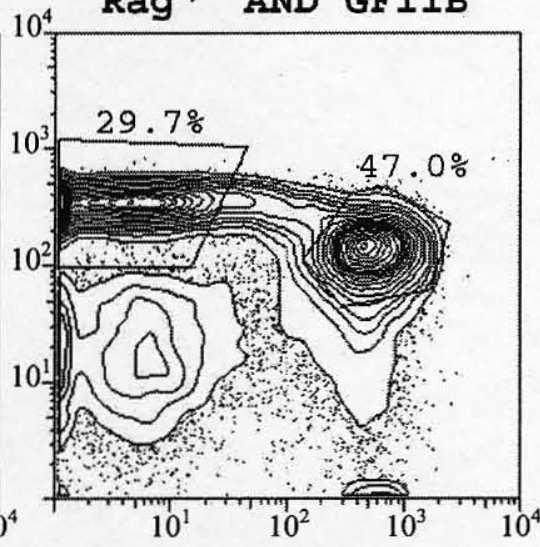

\begin{tabular}{l|c|c|c|c|}
\hline & \multicolumn{2}{|c|}{ Rag $^{*}$ AND $(\mathrm{n}=6)$} & \multicolumn{2}{c|}{ Rag $^{*-}$ AND GFII $(\mathrm{n}=5)$} \\
\hline Cell population & $10^{6}$ cells & S.D. & $10^{\circ}$ cells & S.D. \\
\hline Total thymocytes & 51.21 & 6.009 & 47.89 & 9.229 \\
\hline DP & 15.41 & 3.593 & 24.54 & 5.010 \\
\hline CD4 SP & 25.43 & 2.680 & 11.80 & 2.520 \\
\hline
\end{tabular}

B

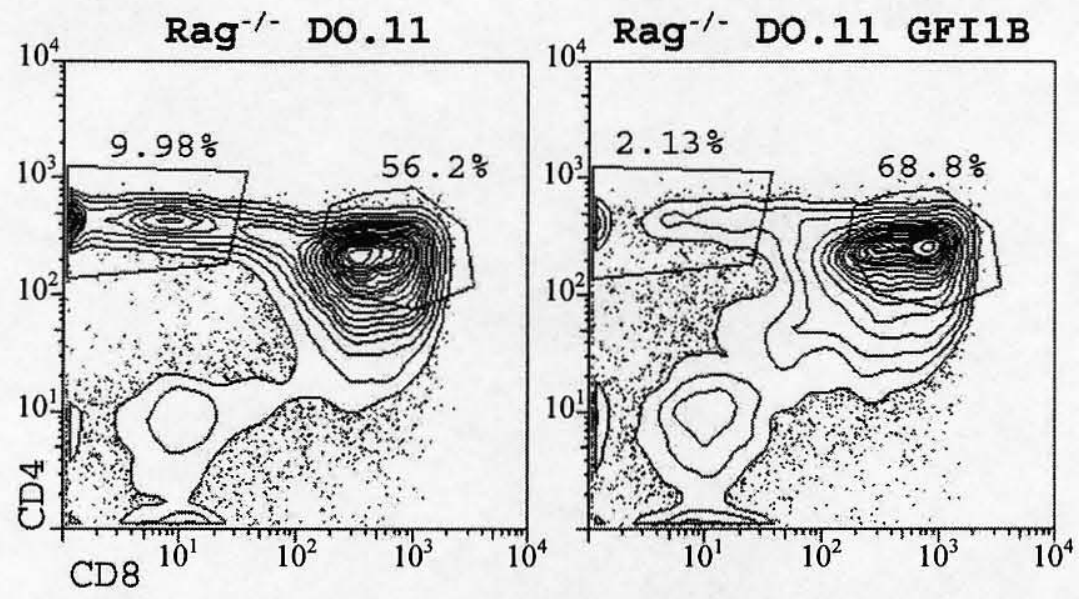

\begin{tabular}{l|c|c|c|c|}
\hline & \multicolumn{2}{|c|}{$\mathrm{Rag}^{7 /} \mathrm{DO} .11(\mathrm{n}=6)$} & \multicolumn{2}{c|}{ Rag DO.11 GFI1B ( $\mathrm{n}=4)$} \\
\hline Cell population & $10^{6} \mathrm{cells}$ & S.D. & $10^{6}$ cells & S.D. \\
\hline Total thymocytes & 216.7 & 13.47 & 246.0 & 16.20 \\
\hline DP & 118.3 & 11.99 & 172.9 & 14.66 \\
\hline CD4 SP & 19.21 & 1.604 & 4.660 & 0.3221 \\
\hline
\end{tabular}


mice are of a mixed haplotype; $d$ (positively selecting) and $b$ (negatively selecting). Therefore, we cannot rule out a role for negative selection in the disparity of CD4 SP in DO.11/GFI1B bi-transgenics, though preliminary data would suggest that GFI1B does not affect negative selection (discussed above). Nonetheless, the results from both Class II restricted transgenic TCR systems are consistent with an interference of positive selection by transgenic GFI1B.

Because we had observed abnormal phenotypically mature CD4 SP cells in GFI1B single transgenic mice (Chapter 2 and Figure 25), we examined the CD4 SP cells in our bi-transgenic system for the expression of several maturation markers. As normal thymocytes mature, they downregulate the expression of CD24 and CD69 (Figure 25). However, GFI1B transgenic mice display an abnormal population of CD4 SP thymocytes that are CD69hi CD24lo (Figure 25). Additionally, normal thymocytes upregulate the expression of CD62L as they mature (data not shown). When considered together, these three markers can be used to track the maturation of thymocytes. The most mature thymocytes are those that express low levels of CD24 and CD69 and high levels of CD62L. These thymocytes are equivalent to immature $\mathrm{T}$ cells, and exit the thymus to take up residence in peripheral lymphoid compartments (87).

CD62L is a homing molecule for mature $\mathrm{T}$ cells that directs them into the peripheral lymphoid compartments (88). The activities of CD24 and CD69 have not been firmly established in T lymphopoiesis; however, it has been suggested that CD69, a glycoprotein, serves as an anatomical anchor, preventing the premature egress of developing thymocytes $(89 ; 90)$. Therefore, in order to exit the thymus and home to peripheral lymphoid compartments, a cell must both downregulate CD69 and upregulate 


\section{Figure 25. GFI1B transgenic CD4 SP thymocytes display abnormal levels of}

maturation markers. Shown are plots of CD4 SP cells from one wild type and one GFI1B transgenic mouse. The GFI1B transgenics have an abnormal population of cells expressing low levels of CD24, most of which express high levels of CD69. In addition, GFI1B transgenics lack a substantial $\mathrm{CD} 24^{\mathrm{int}} \mathrm{CD} 69^{\mathrm{lo}}$ population. 
Figure 25
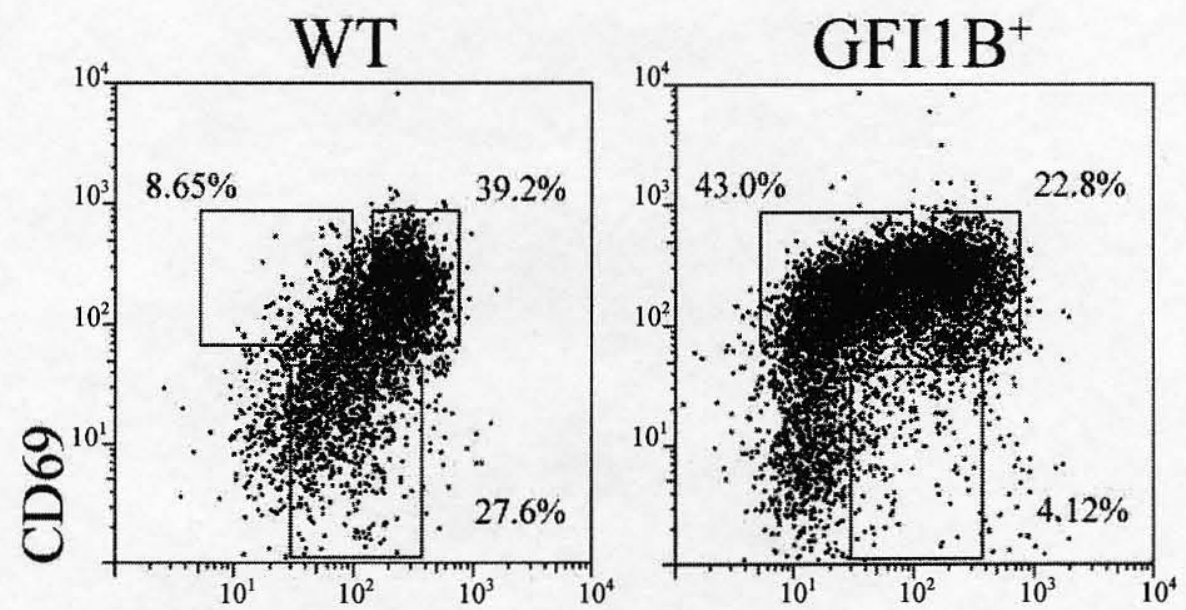

CD24 
CD62L. The increase in CD4 SP cells that we observed in our GFI1B transgenic mice was comprised of cells that express low levels of CD24 (Figure 10C), suggesting that these cells were phenotypically mature. However, these cells also express high levels of CD69 (data not shown), suggesting abnormal maturation. We therefore examined the CD4 SP cells generated in our GFI1B/TCR bi-transgenic systems for maturity based on CD24, CD69, and CD62L expression.

As shown in Figure 26, GFI1B ${ }^{-}$TCR transgenic CD4 SP cells are mostly CD24 ${ }^{\text {hi }}$ $\mathrm{CD} 69^{\text {hi }}$, with a relatively abundant population expressing intermediate levels of both markers, suggesting a normal maturation process. Furthermore, a good percentage of these cells express CD62L, suggesting phenotypically mature thymocytes with the capacity to exit the thymus and home to the periphery. In contrast, GFI1B ${ }^{+} \mathrm{TCR}$ transgenic CD4 SP cells are largely $\mathrm{CD} 24^{\mathrm{lo}} \mathrm{CD} 69^{\mathrm{hi}}$, suggesting abnormal maturation. In addition, very few GFI1B transgenic CD4 SP cells express CD62L. Therefore, though GFI1B transgenic mice generate CD4 SP cells with certain markers of mature thymocytes, these cells may be unable to exit to the periphery and home to lymphoid compartments due to continued expression of CD69 and a lack of expression of CD62L, respectively.

\section{Microarray analysis reveals alterations in expression of several genes involved in}

\section{TCR signaling pathways in GFI1B transgenic thymocytes.}

We hypothesize that GFI1B interferes with TCR-induced signaling, resulting in a decrease in the strength of signal that the cell interprets. Much is known about the pathways involved in, and the ultimate downstream effectors of, TCR-induced signals. Figure 27 is a simplified schematic representation of some of the major players in three 
Figure 26. CD4 SP cells of TCR GFI1B bi-transgenic mice do not mature normally. A. CD69 or CD62L v CD24 in CD4 SP thymocytes of Rag ${ }^{-/-}$AND TCR transgenic or GFI1B littermates. Percentages of gated populations are indicated. B. The same analyses applied to $\mathrm{Rag}^{-/-}$DO.11 TCR transgenic mice. 
Figure 26

A
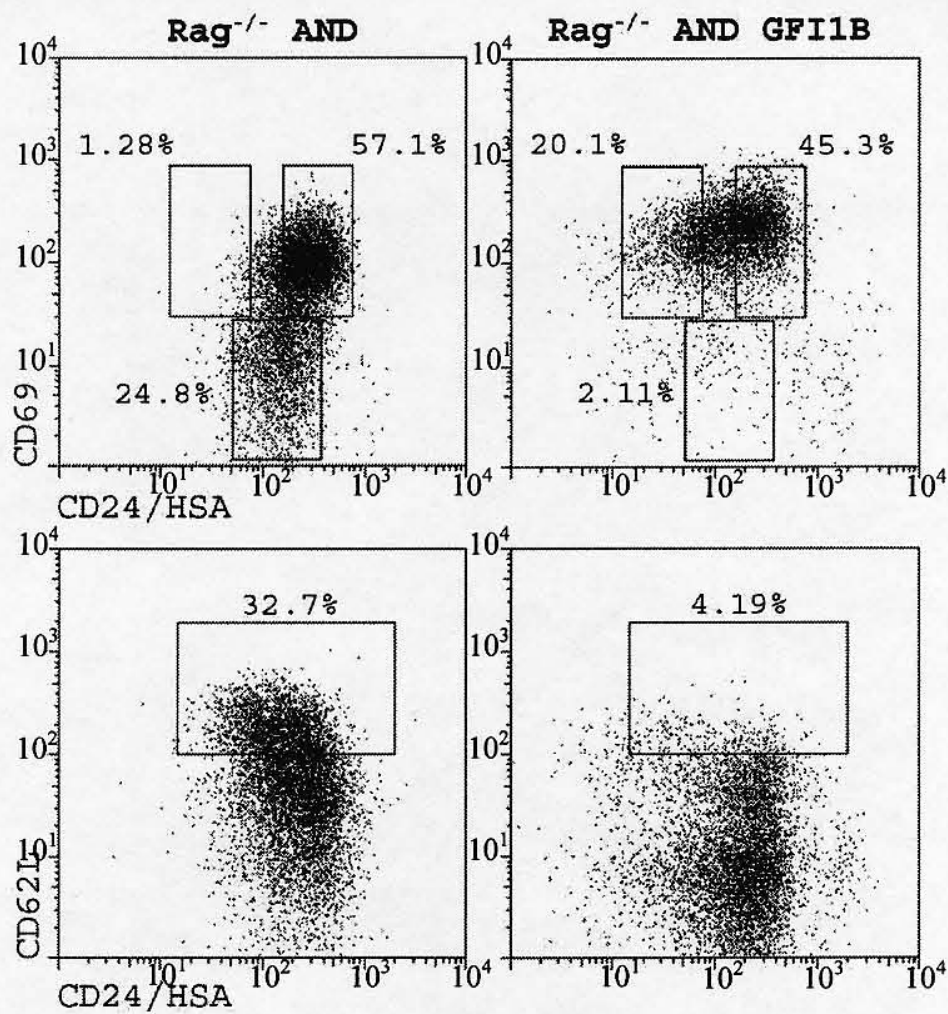

B
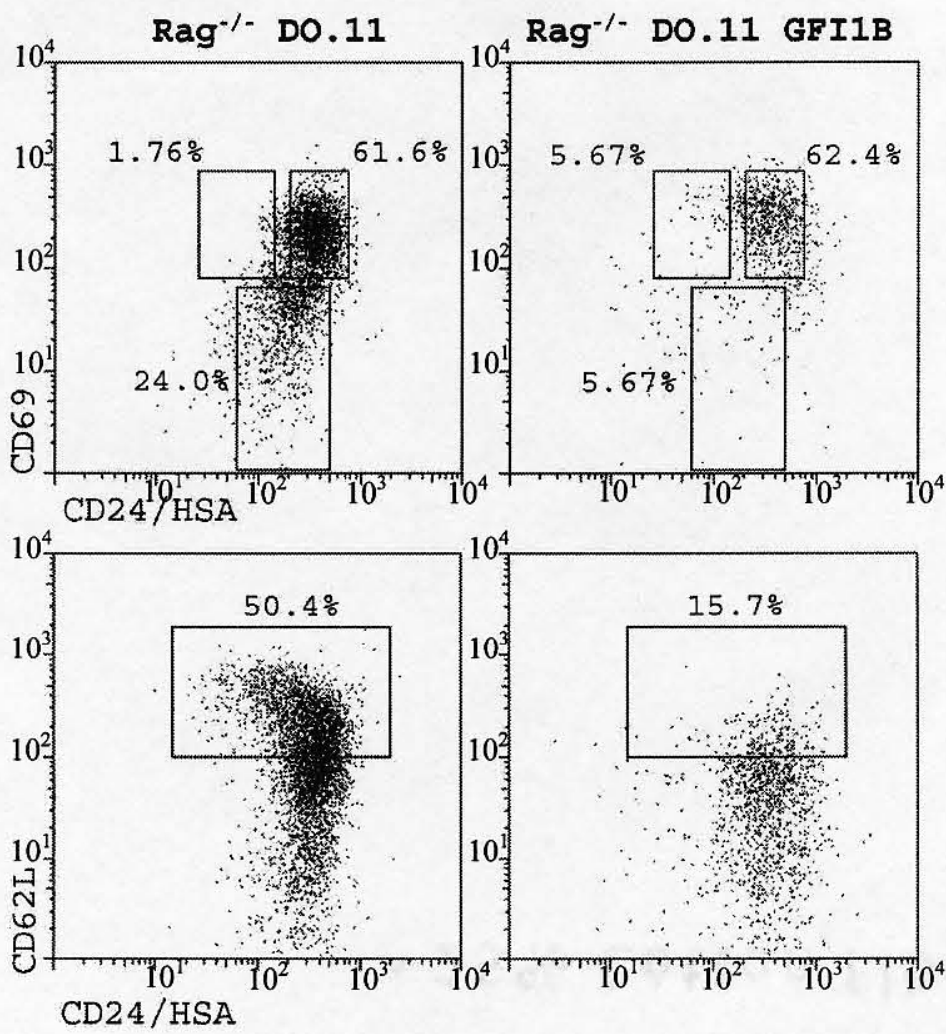
Figure 27. Schematic representation of major effectors in TCR signaling pathways. Modified from Singer and Koretzky, STKE website. The three major pathways depicted result in the transcriptional activation of three different factors. Activation of both NF$\kappa \mathrm{B}$ and $\mathrm{AP}-1$ requires signaling through the second messenger diacylglycerol (DAG). However, the activation of NF- $\mathrm{kB}$ is PKC-dependent whereas the activation of AP-1 is mediated through the Ras pathway. Activation of N-FAT is mediated through the calcium/calcineurin pathway. http://stke.sciencemag.org/content/vol0/issue2003/images/data/CMP 7019/DC1/Singer4_2003.jpeg 
Figure 27

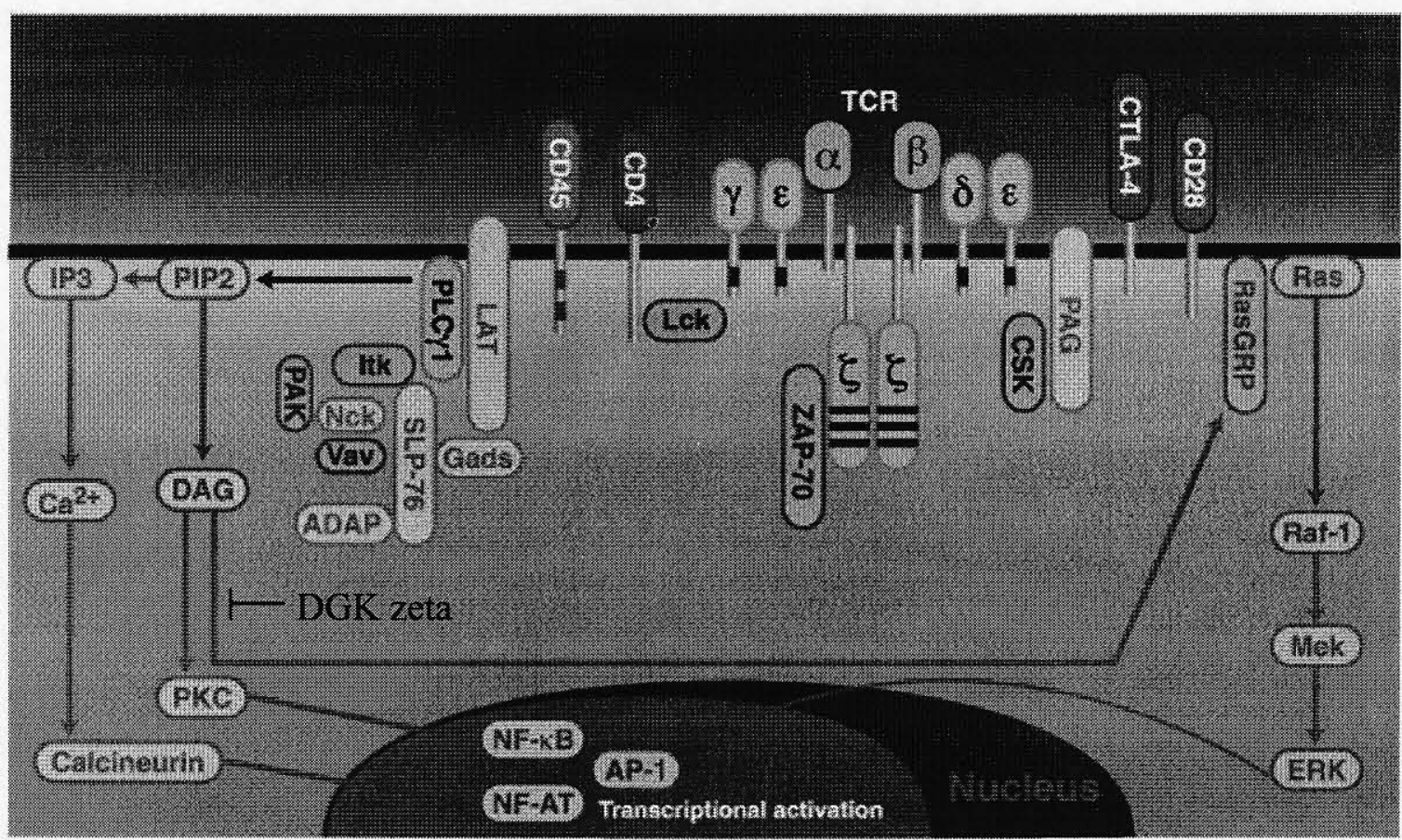


distinct TCR signaling pathways. Each pathway activates a distinct downstream effector. That is, calcium/calcineurin-mediated signaling results in the activation of Nuclear Factor of Activate T-cells (N-FAT), whereas PKC-dependent signaling results in an increase in the transcriptional activation of Nuclear Factor kappa $\mathrm{B}(\mathrm{NF \kappa B})$, and signaling through the Ras pathway induces activation of AP-1, the Fos/Jun family of transcriptional activators. As all of these are required for a full response to TCR activation, interference with any of them would result in a decrease in that response. We therefore performed microarray analysis on TCR/GFI1B bi-transgenic thymi and examined the expression of known members of these pathways.

Figure 28 is a table of absolute signals from our microarray analysis. Included in the table are genes involved in TCR signaling that we found to be different between GFI1B $^{+}$and GFI1B ${ }^{-}$mice. We first examined the expression of $G f i l$, which we have shown to be repressed by GFI1B, and of GfilB itself, as we would expect to see increased levels of GfilB transcript in GFI1B transgenic cells. Indeed, GFI1B transgenic thymocytes express drastically reduced levels of $G f i l$ message and increased levels of GfilB transcript when compared to littermate controls (Figure 28). Furthermore, GFI1B transgenics express increased levels of diacylglycerol kinase zeta $(D G K \zeta)$, a negative regulator of diacylglycerol (DAG) signaling. As depicted in Figure 27, this increase alone may be sufficient to decrease the activation of both NFkB and AP-1 upon induction of TCR signals. Additionally, GFI1B transgenics express decreased levels of both $P K C \theta$ and RasGRP2, further contributing to the block in activation of NFKB and AP-1, respectively. These results must be confirmed by either Northern blot analysis or RTPCR. However, in order to justify further investigation into this potential mechanism, we 
Figure 28. GFI1B transgenic thymocytes display abnormal expression of transcripts relevant to TCR signaling pathways. Signals of Affymetrix MOE430 microarray of RNA in thymocytes from two GFI1B/AND bi-transgenic and two GFI1BAND TCR transgenic littermate controls. In GFI1B transgenic animals, Gfil transcript is reduced, and GfilB transcript is increased, when compared to GFI1B- littermate controls. The array has two different probe sets to examine $D G K \zeta$; both are listed. Though absolute values for GFI1B transgenic animals are not always higher than controls, the trend is that GFI1B transgenic thymocytes have increased levels of $D G K \zeta$ message. The signal for $P K C \theta$ is lower in the single probe set for that gene, and all three probe sets for RasGRP2 show decreased levels of this transcript in the GFI1B transgenics. Signal values were normalized using global scaling to a constant factor to compensate for variation in sample processing. 
Figure 28

\begin{tabular}{|l|r|r|r|r|}
\hline $\begin{array}{l}\text { AND Tg } \\
\text { GFI1B Tg }\end{array}$ & \multicolumn{1}{|c|}{+} & $\begin{array}{c}+ \\
-\end{array}$ & $\begin{array}{c}+ \\
+\end{array}$ & $\begin{array}{c}+ \\
+\end{array}$ \\
\hline Gfi1 & 288.8 & 367.4 & 61.5 & 13.0 \\
\hline Gfi1B & 9.5 & 7.8 & 904.5 & 1036.9 \\
\hline DGK zeta & 2312.9 & 3067.9 & 3979.9 & 3059.6 \\
\hline DGK zeta & 299.6 & 521.5 & 834.5 & 742.3 \\
\hline PKC theta & 796.5 & 706.8 & 369.6 & 455.2 \\
\hline RasGRP2 & 127.1 & 168.3 & 93.3 & 93.0 \\
\hline RasGRP2 & 116.2 & 152.5 & 102.1 & 83.5 \\
\hline RasGRP2 & 96.8 & 79.8 & 57.6 & 66.1 \\
\hline
\end{tabular}


needed preliminary evidence that the downstream effectors of these signaling pathways are also affected in the GFI1B transgenics.

\section{EMSA analysis suggests that NFkB and AP-1 are less active in GFI1B transgenic}

\section{thymocytes.}

$\mathrm{NFKB}$ and $\mathrm{AP}-1$ are activated in response to TCR signals, and this activation is often measured by examination of DNA-binding capacity of these factors in nuclear extracts. We therefore performed EMSA analysis to compare the activity of these transcription factors in GFI1B transgenic thymocytes and GFI1B negative littermate controls. As a control for equal nuclear extract loading, we included probes for C/EBP, which is not responsive to TCR activation. Figure 29 shows the results of this analysis for both AND and DO.11 TCR transgenics. In AND TCR transgenics, expression of transgenic GFI1B results in a decrease in DNA binding by both $\mathrm{NFKB}$ and $\mathrm{AP}-1$, but does not affect DNA binding by C/EBP (Panel A). These results are consistent with the gene array data in which we observed increased expression of $D g k \zeta$ and decreased expression of both $P K C \theta$ and RasGRP2e (Figure 28). Examination of the DNA binding activity of these same transcription factors from DO.11 GFI1B transgenic thymocytes revealed decreased activity of NFKB, but not of AP-1 or C/EBP. These preliminary results, while far from conclusive, provide biological impetus to continue exploring the effects of GFI1B on both PKC- and Ras-mediated TCR signaling.

Future experiments to determine whether these pathways are affected in the GFI1B-mediated block to positive selection include Northern blot or RT-PCR analysis as previously mentioned. In addition, promoter and ChIP analyses would help to determine whether the decreased expression of $P K C \theta$ and $\operatorname{Ras} G R P 2$ are the result of direct 
Figure 29. Transgenic GFI1B decreases DNA binding by TCR downstream

effectors in bulk thymocytes. A. EMSA analysis of DNA binding by NFKB, AP-1, and $\mathrm{C} / \mathrm{EBP}$ in thymocyte nuclear extract from $\mathrm{Rag}^{-/-}$AND TCR GFI1B bi-transgenic and littermate controls. Transgenic GFI1B results in decreased intensities of shifts for both NFkB and AP-1, but not for C/EBP. B. The same analyses applied to nuclear extract from a different transgenic TCR $\left(\mathrm{Rag}^{-/} \mathrm{DO} .11 \mathrm{TCR}\right.$ transgenic mice) reveals decreased binding by NFאB, but not by AP-1 or C/EBP. 
Figure 29
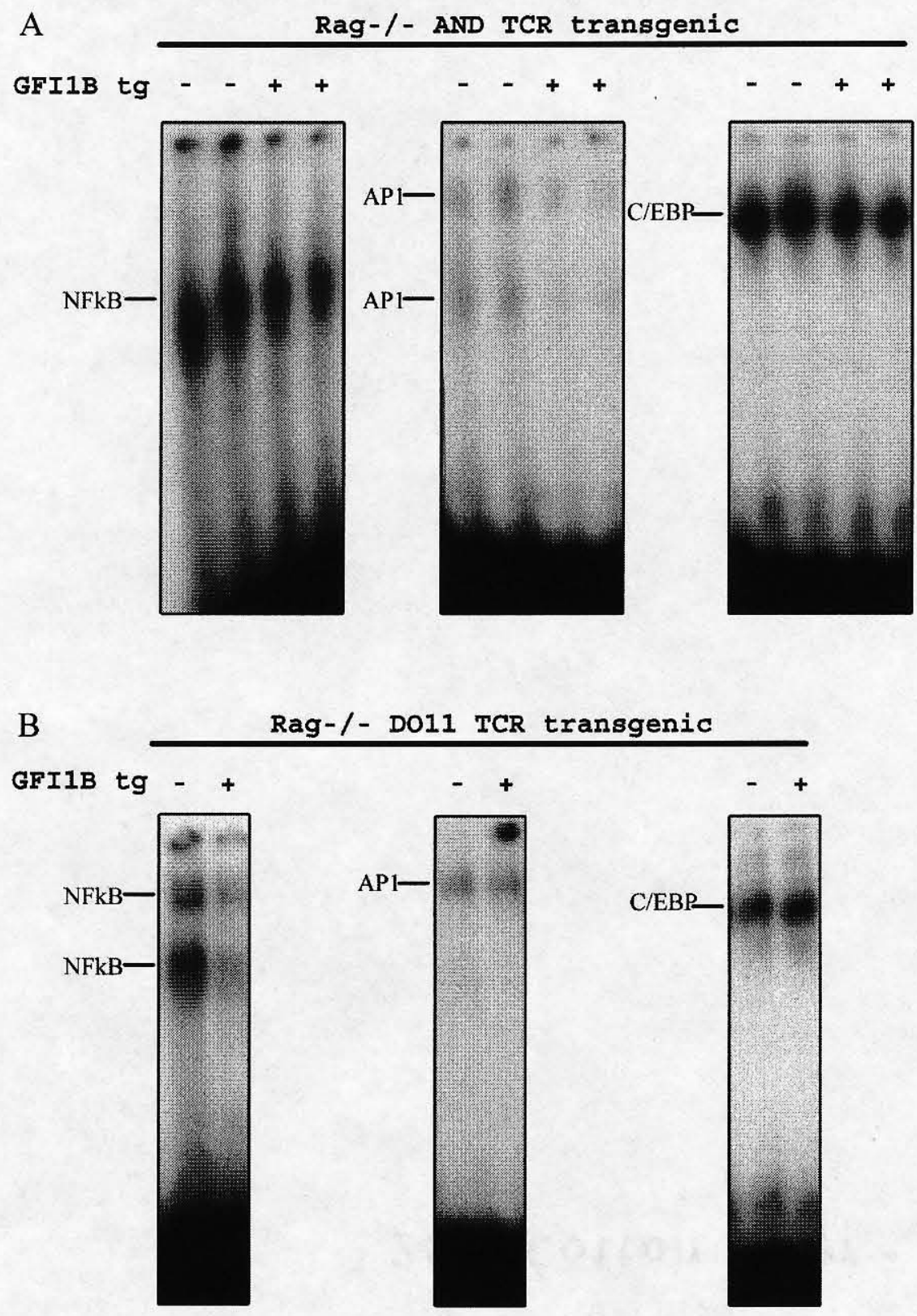
repression by GFI1B. Expanding on the results obtained by EMSA, the same analyses should be repeated on sorted DP TCR ${ }^{\text {hi }}$ thymocytes (the stage at which positive selection occurs and endogenous $G f i 1 B$ is expressed) to determine whether in this population, the activities of NFkB and AP-1 are truly decreased. Additionally, TCR transgenic thymocytes could be activated and the same analyses performed to further support the theory that this is a TCR-mediated inducible effect. In sum, the data presented here are preliminary and provide a solid framework and good starting point for future work investigating the mechanisms by which GFI1B decreases positive selection. 


\section{CHAPTER VI \\ CLOSING REMARKS}

The T lymphopoietic process is of great interest for many reasons. Not only has the study of this developmental pathway led to a greater understanding of immunity and autoimmunity, but it is also a good model for studying developmental processes in general. While most development ceases after embryogenesis, $\mathrm{T}$ lymphopoiesis continues into adulthood, albeit at a slower rate. Furthermore, and perhaps most pertinent in the context of these studies, there is a close relationship between development and oncogenesis. That is, many of the factors that regulate development also drive the oncogenic process (recent reviews include (91-94)).

The transcriptional repressors GFI1 and GFI1B have been identified as oncogenes and perform defined oncogenic functions. Indeed, these factors were originally identified in Moloney Murine Leukemia Virus-induced leukemia systems, and were characterized as being able to block growth arrest, differentiation, and apoptosis $(8-10 ; 31 ; 62 ; 76)$. Sequence analysis of $G f i 1$ and $G f i 1 B$ revealed extensive homology in both the N-terminal region, which encodes the transcriptional repressor SNAG domain, and in the C-terminal region encoding six Cis-His $\mathrm{Zn}$ fingers (Figure 3) (10). Furthermore, in vitro assays revealed that GFI1 and GFI1B bind to nearly identical recognition sequences and repress transcription in a SNAG-dependent manner $(9 ; 10 ; 15)$. However, Gfil and GfilB showed 
tissue-specific expression patterns (10), leaving an apparent dichotomy; if these two factors are biochemically and functionally redundant, why are there two differentially expressed proteins?

This work was initiated to address the question of functional redundancy between GFI1 and GFI1B. We chose to examine the effects of transgenic expression of GFI1 or GFI1B in T cells because GFI1 had already been shown to have a biological function in these cells. During the course of our work, other literature has also emerged examining the effects of either transgenic expression of GFI1 or deficiency of either Gfil or GfilB. What has emerged from the work done to date is a story of two unique factors, each having distinct roles in development. Gfil is essential for normal T lymphopoiesis and granulopoiesis, while $G f i 1 B$ is required for definitive erythropoiesis $(11 ; 16-18)$. The published findings of the studies producing these data have been mentioned in more detail throughout the earlier chapters of this dissertation, and will not be discussed further here. Instead, this chapter will focus on the distinct roles of GFI1 and GFI1B in positive selection of developing thymocytes, drawing both from a body of published literature and from the data generated in this lab and presented in this dissertation.

Based on early biochemical studies, we began this work with the hypothesis that, if expressed independently in the same tissue and in the same manner, GFI1 and GFI1B may perform redundant functions. To test this hypothesis, mice were generated to express transgenic GFI1 or GFI1B in developing and mature T cells. One of our first observations of GFI1 transgenic $\mathrm{T}$ cells is that they respond more robustly to activation through the TCR (Figure 8), a result that has been corroborated by others (21). On the contrary, the same analysis applied to GFI1B transgenic T cells yielded a directly 
opposite result. That is, GFI1B-transgenic T cells are defective in activation (Figure 8). This was perhaps surprising at first, given the biochemical similarities of GFIl and GFI1B. However, the opposing functions of these two factors in T cells may have been suggested by very early studies in which GFI1B was found to be up-regulated in Moloney Murine Leukemia Virus (MoMuLV)-induced B cell tumors, but not in tumors of T cell origin (10). Since T cells require TCR signals to survive, the lack of GFI1B in these tumors may have been a clue to the detrimental effect of GFI1B on TCR-mediated activation.

Our data suggest that GFI1B negatively affects normal positive selection of thymocytes by interrupting TCR signaling events. GFI1 is required for normal T lymphopoiesis $(11 ; 16)$. Thymi of our GFI1 and GFI1B transgenic mice have different phenotypes. Our GFI1 transgenic system failed to reproduce the phenotype observed in an earlier publication (36), a result that will be discussed in more detail below. However, our GFI1B transgenics displayed abnormal thymocyte development, manifested by an increase in the $\mathrm{CD} 4 / \mathrm{CD} 8$ ratio and a decrease in the expression of the late activation marker IL7R $\alpha$. Furthermore, in two independent mouse models that express specific MHC Class II restricted TCRs, the expression of transgenic GFI1B resulted in a decrease in the number of CD4 SP cells. This result suggests that overexpression of GFI1B interferes with positive selection of DP thymocytes. In addition, our data show that GfilB is up-regulated in thymocytes that are undergoing positive selection events. Considered together with the GFI1 literature and the effects of GFI1 and GFI1B on T cell activation, our data implicate GFI1 and GFI1B in TCR signaling. 
I propose a model of $\mathrm{T}$ lymphopoiesis in which GFI1 enhances, while GFI1B opposes, positive selection. I propose that these opposite effects are engendered by at least two mechanisms. One mechanism is the distinct activities of GFI1 and GFI1B that result from their unique intervening sequences. The other mechanism is direct repression of Gfil by GFI1B at the DP stage during which TCR signaling must be fine-tuned in order for proper selection to occur. (See Figure 30.) Furthermore, I propose that these effects can be observed at earlier stages of thymocyte development, a concept that will be expanded below.

Evidence for the stimulatory effect of GFI1 on positive selection comes from the study of GFI1 transgenic mice. First, as mentioned throughout this dissertation as a fundamental finding, GFI1 transgenic T cells are more responsive to TCR stimulation than are wild type T cells. TCR signaling is required for positive selection. This requirement raises the possibility that enhanced signaling may result in enhanced selection. Conversely, because an overly strong signal results in negative selection, it remains possible that by enhancing TCR signaling, GFI1 could actually increase negative selection as opposed to positive selection, resulting in an overall decrease in the production of SP thymocytes. However, in one published report, GFI1 transgenic thymi, though drastically reduced in overall cell number, showed an increase in the percentage of SP thymocytes and a decrease in the percentage of DP thymocytes when compared to littermate controls (36). This increase occurred with no consistent bias toward either the CD4 or CD8 lineage. A simple precursor-progeny ratio, then, would suggest that GFI1 does indeed enhance the positive selection of DP thymocytes. 
Evidence for the deleterious effect of GFI1B on positive selection has been presented in chapters 3 and 5 of this dissertation. Our data show that in isolation, transgenic expression of GFI1B results in an abnormally matured population of CD4 SP cells and a decrease in the number of CD8 SP cells that are generated. The lack of CD8 SP cells in GFI1B transgenic mice suggested defective positive selection, but was made less precise in light of the increased numbers of CD4 SP cells. However, we hypothesized that the increased number of CD4 SP cells in the GFI1B transgenics is itself the result of decreased TCR signals. This aberrant signaling leads to the positive selection of cells that should have been eliminated. These abnormal cells are then unable to develop further into functional immune cells and exit the thymus. Indeed, in the restricted selecting environment engendered by expression of a Class II-restricted transgenic TCR, transgenic GFI1B decreases the number of CD4 SP cells that are generated, with a concomitant increase in the DP population. Again, precursor-progeny relationship would suggest a defect in positive selection. Therefore, it seems logical that, due to a lack of proper TCR and/or IL7 signaling, GFI1B transgenic thymocytes are at a disadvantage for survival.

The decrease in CD8 SP cells observed in GFI1B single transgenic mice has been tied both to decreased expression of IL7R $\alpha$ and to a lack of GFI1 expression. The partial restoration of this population by forced expression of GFI1 not only emphasizes the effects of GFI1B-mediated repression of Gfil, but also places GFI1 uniquely in the path of CD8 SP development and further supports a role for GFI1 in survival and positive selection. Furthermore, these data highlight the distinct roles of GFI1 and GFI1B in thymocyte development. The most straightforward explanation for the results is that 
Figure 30. Schematic representation of GFI1 and GFI1B in positive selection of DP thymocytes. A. The effect of overexpression of GFIl. Too much GFIl results in increased positive selection and an imbalance of SP to DP cells, in the direction of SP. The region between the SNAG domain and the $\mathrm{Zn}$ fingers is encircled and is likely to mediate this GFI1-specific effect. B. The effect of overexpression of GFI1B. Too much GFI1B results decreased positive selection and an imbalance of SP to DP cells in the direction of DP. Both SNAG-dependent complete repression of Gfi1 and SNAGindependent GFI1B-specific functions contribute to this effect. The unique region of GFI1B is encircled. C. GFI1 and GFI1B in normal positive selection. Moderate expression of GFI1B results in moderate repression of Gfi1 and proper levels of GFI1 protein. Thus, both factors are present to contribute unique functions and maintain proper balance of signals, leading to normal positive selection. 
A

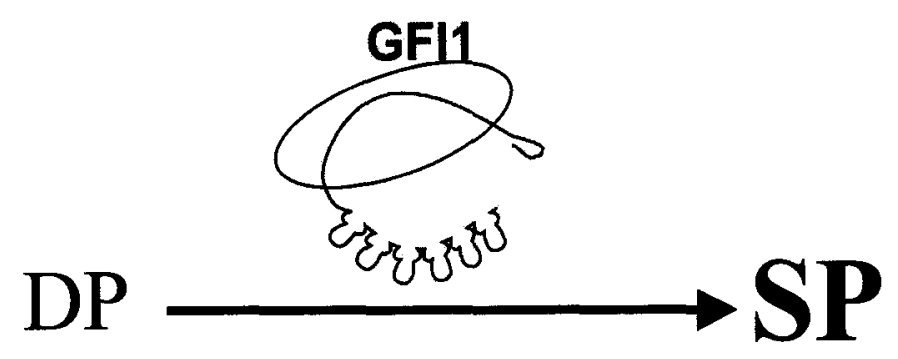

B

GFI1B

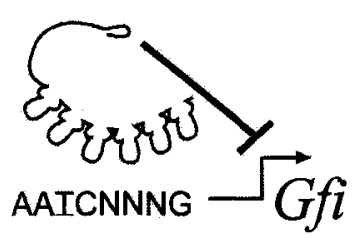

$\mathrm{DP} \longrightarrow \mathrm{SP}$

C

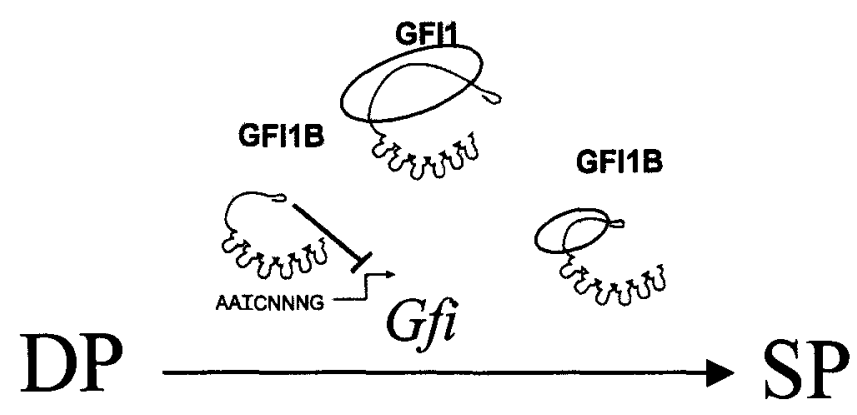


the unique region of GFI1 is responsible for supplying the survival signal necessary for CD8 SP formation, which GFI1B is unable to provide. It is interesting to consider that GFI1 has been shown to physically interact with Protein Inhibitor of Activated STAT (PIAS)3, an inhibitor of Signal Transducer of Activated T cells (STAT)3, through this unique region. STAT3 has been shown to transduce signals through both IL2 and IL6 pathways in T cells, although a function for STAT3 in T lymphopoiesis has not been defined. The interaction of GFI1 with PIAS3, and possible implications thereof, are discussed in further detail below. However, though strictly correlative, these data suggest a role for GFI1-mediated STAT3 activation in the generation and selection of CD8 SP thymocytes.

The most novel data presented in this dissertation are those that predict a role for GFI1B in normal T lymphopoiesis. In addition to the evidence gleaned from transgenic mouse models, we and our collaborators have provided the first evidence that GFI1B is expressed in normal developing thymocytes (Figure 6). This physiological expression of GfilB at stages of positive selection provides insight into the function of GFI1B in T cells. Furthermore, our data showing repression of $G f i 1$ by GFI1B begins to elucidate a partial mechanism for the action of GFI1B in T cells; to specifically repress the transcription of the gene encoding the signal-enhancing factor GFI1, thereby providing a damper of TCR signaling in cells poised to differentiate into the SP stage. However, I do not suggest that all of the functions of GFI1B are dependent upon its repression of Gfil. Indeed, we have provided evidence to the contrary in that transgenic GFI1 does not correct all of the phenotypes engendered by transgenic expression of GFI1B. However, it 
remains possible that the inability of transgenic GFIl to reverse all phenotypes of transgenic GFI1B is a result of inadequate expression of the transgene.

At least two other possible mechanisms could contribute to GFIlB's function in T cells. The first is direct competition with GFI1 for specific target genes that are differentially regulated by GFI1 and GFI1B. The other is unique GFI1B-specific interaction with other proteins that have functions in signal transduction. In fact, though distinct in execution, each of these proposed mechanisms is dictated by the unique nonhomologous central regions of GFI1 and GFI1B.

The first proposed molecular mechanism of GFI1B antagonism of GFI1 function in T cells is the direct competition of GFI1 and GFI1B for binding to target genes. For antagonistic functions to result from one or the other factor binding preferentially to target genes, the genes in question must be differentially regulated by GFI1 and GFI1B. This differential regulation must be mediated by the non-SNAG or $\mathrm{Zn}$ finger regions of the two proteins. These non-homologous regions may provide different protein-protein interaction domains that would interact with different transcription factors or co-repressor (or co-activator) proteins, thereby providing the basis for differential regulation. These interactions could in fact influence the DNA binding of GFI1 and GFI1B, or the distinct nature of the interactions may change the context of transcriptional control after DNA binding. Either mechanism could result in opposing outcomes dependent on the presence of either GFI1 or GFI1B.

Interaction with different binding partners could alter the DNA binding specificities of GFI1 and GFI1B. It can be assumed that these two factors normally exist in a complex with other proteins, which would bind to DNA as a unit. Our EMSA data 
would suggest that this is true for GFI1 (Figure 21). In this proposed mechanism, the components necessary for interaction are complexed together before being recruited to DNA. Therefore, if GFI1 and GFI1B are part of pre-existing complexes before binding to DNA, the presence of the interacting partners could alter the DNA binding of GFI1 and GFI1B in at least two ways. If the interacting partner is itself a transcription factor with a distinct DNA recognition sequence, it is likely to change the affinity of GFI1 or GFI1B binding by itself making contact with the DNA in the presence of its recognition element. This interaction would in turn stabilize the overall protein-DNA interaction and increase the apparent affinity of GFI1 or GFI1B for its binding site. Alternatively, the GFI1- or GFI1B-interacting proteins present in the pre-formed nuclear complex may not bind to DNA at all. Rather, they could impose a slight conformational change in GFI1 or GFI1B, thereby slightly altering the affinity for binding. The non-homologous nature of the intervening sequences of GFI1 and GFI1B suggest distinct protein-protein interactions and unique binding partners. Therefore, the mechanisms discussed above would confer differential regulation of target genes in at least two ways. First, binding affinities of GFI1 and GFI1B for specific sites would be different. Second, the unique complexes would likely provide distinct regulation of the target genes.

Another model to consider is transcriptional regulation in the context of a repressosome. Repressosomes and enhanceosomes are nucleoprotein complexes that assemble on regulatory regions of DNA to regulate the transcription of genes (recently reviewed in (95-97)). The success of a repressosome depends on the DNA recognition sequences of specific transcription factors and on the presence of the factors themselves. Furthermore, proper binding of the DNA-interacting factors specifies the conformation 
for the recruitment of specific co-repressors. When all components are present, the repressosome is assembled into a three dimensional structure that often involves bending of DNA to bring transcription factors into close proximity to one another, allowing necessary protein-protein interactions. Therefore, in this very complex system, the composition of molecular species is very tightly controlled. That is, incorporation of a non-functional factor, or perhaps the wrong factor, would result in interruption of the protein-protein interactions that ensure proper repressosome formation and subsequent transcriptional regulation by the repressosome complex. The intervening sequences of GFI1 and GFI1B are dissimilar and therefore would not be predicted to interact with similar proteins. Thus, if GFI1B were to bind to a recognition site that is part of a GFI1requiring repressosome, then the final transcriptional regulation would not be correct. This theory, again, depends on the non-homologous regions of GFI1 and GFI1B to confer the differences. However, this model is more likely to be relevant in situations where one or the other factor is aberrantly overexpressed, as the binding of the correct factor in the repressosome would stabilize the tertiary structure. This stabilization would energetically favor the binding of the correct factor such that this would be the prevalent composition unless enough of the incorrect factor was present to shift the equilibrium of binding.

The second proposed molecular mechanism is quite straightforward, and therefore very attractive to consider, though there is very little evidence to support it as yet. GFI1 has been shown to confer signaling advantages to cells by increasing the activity of Signal Transducer of Activated T cells (STAT)3. GFIl does this by interacting with, and interrupting the function of, Protein Inhibitor of Activated STAT (PIAS)3 (20). PIAS3 inhibits the DNA binding capability of activated STAT3 by binding to and sequestering 
the active STAT3 dimer (98). However, PIAS3 has also been shown to have other effects in the cell, including mediation of sumoylation through interaction with SUMO-1 $(99 ; 100)$. SUMO is a small molecule that resembles ubiquitin and is conjugated to proteins in a process similar to ubiquitination. However, sumoylation often occurs on transcription factors in the nucleus, and does not lead to proteasomal degradation. Rather, sumoylation regulates the subnuclear localization and activity of the target transcription factors (101). Therefore, GFI1-PIAS3 interaction may signify alterations in GFI1's transcriptional activity in addition to interference with STAT3 activity.

The interaction of GFI1 with PIAS3 has been mapped to the region between the SNAG domain and the $\mathrm{Zn}$ fingers (20), which shares no homology with the same region of GFI1B. Therefore, the unique region of GFIl can act to sequester signaling proteins and change the context of signaling pathways independent of any direct transcriptional repression. It is interesting to consider that PIAS3 is likely only one of several proteins with which GFIl can interact, and that GFI1B, due to having no homology to GFI1 in this region, at the very least does not interact with the same signal-enhancing proteins as does GFI1 and may indeed interact with different proteins to confer opposite effects on signal transduction.

These same principles may be in place earlier in T lymphopoiesis. GFI1 has been suggested to play a role in early thymocyte development at the stage of beta selection, which occurs at the DN3 stage of thymocyte development $(11 ; 36)$. Our data have shown that GfilB is up-regulated at this stage of development. It is therefore possible that, similar to its effect on TCR signaling, GFI1B decreases pre-TCR-mediated signals to influence these early maturation steps. However, our GFI1 transgenics did not reproduce 
the published phenotype, nor did we observe an effect of transgenic GFI1B at this stage. Therefore, the roles of GFI1 and GFI1B in beta selection cannot be clearly defined at this point. Nonetheless, there does seem to be a function for GFI1 prior to beta selection, as determined by examination of Gfil deficient mice and corroborated by data from our GFI1B transgenics.

Mice that are deficient for Gfil display a severe block to T lymphopoiesis in the very early DN1 to DN2 transition (11). Our expression data corroborate a role for GFI1 at this step in that we observe an approximately 10-fold increase in Gfil message levels in DN2 versus DN1 cells (Figure 6). This increase in expression suggests a requirement for the presence of GFI1 in this transition, and supports the result that lack of Gfil causes a block in early thymocyte development. Furthermore, GFI1 transgenic mice have a larger percentage of both $\alpha / \beta$ TCR-expressing and $\gamma / \delta$ TCR-expressing cells in total thymocyte populations (36). While the increase in $\alpha / \beta$ cells may be attributed to the previously mentioned increase in the percentage of SP thymocytes, the increased percentage of $\gamma / \delta$ T cells should be manifested in the DN population, as $\gamma / \delta$ lineage commitment occurs prior to expression of either CD4 or CD8. Furthermore, normal $\gamma / \delta$ T cells do not express either co-receptor. We observed a similar result in our GFI1B transgenic mice. Upon examination of DN thymocytes, we discovered a reproducible marked increase in the proportion of cells that express either TCR (data not shown). Since both overexpression models show similar results, it is interesting to contemplate that the effect of GFI1 and GFI1B in early T lineage commitment steps is SNAG and Zn finger dependent. In normal thymocytes, only $G f i l$ is expressed at these very early stages, further suggesting redundancy of these two factors at this stage. Again, these data 
are merely suggestive, and require more specific experiments to finally answer the question of the role of GFI1 in early T lineage commitment.

GFI1 and GFI1B, in addition to being potent oncogenic factors, are important regulators of development. The normal physiological functions of these two proteins are just beginning to be understood. The dramatic abnormalities engendered by a complete, unconditional loss of either factor make gene-targeted mouse systems less than ideal for studying the subtle nuances of temporal or conditional changes in expression, such that are likely to be encountered in disease states. Work in the near future is likely to include conditional knockout systems in which loss of Gfil or GfilB can be directed to specific cells at specific stages of development. Such experiments in developing T lymphocytes would clarify some of the finer points of control that these two fascinating transcription factors mediate during the process of $\mathrm{T}$ lymphopoiesis. 


\section{REFERENCES}

1. Love PE, Chan AC. Regulation of thymocyte development: only the meek survive. Curr.Opin.Immunol 2003; 15: 199.

2. Spits H. Development of alphabeta T cells in the human thymus. Nat.Rev.Immunol 2002; 2: 760.

3. Peschon JJ, Morrissey PJ, Grabstein KH, et al. Early lymphocyte expansion is severely impaired in interleukin 7 receptor-deficient mice. J Exp Med 1994; 180: 1955.

4. Carleton M, Haks MC, Smeele SA, et al. Early growth response transcription factors are required for development of $\mathrm{CD} 4(-) \mathrm{CD} 8(-)$ thymocytes to the CD4(+)CD8(+) stage. J Immunol 2002; 168: 1649.

5. Pear WS, Radtke F. Notch signaling in lymphopoiesis. Semin.Immunol 2003; 15 : 69.

6. Greenbaum S, Zhuang Y. Regulation of early lymphocyte development by E2A family proteins. Semin.Immunol 2002; 14: 405. 
7. Macian F, Lopez-Rodriguez C, Rao A. Partners in transcription: NFAT and AP-1. Oncogene 2001; 20: 2476.

8. Gilks CB, Bear SE, Grimes HL, Tsichlis PN. Progression of interleukin-2 (IL-2)dependent rat $\mathrm{T}$ cell lymphoma lines to IL-2-independent growth following activation of a gene (Gfi-1) encoding a novel zinc finger protein. Mol Cell Biol 1993; 13: 1759.

9. Grimes HL, Chan TO, Zweidler-McKay PA, Tong B, Tsichlis PN. The Gfi-1 proto-oncoprotein contains a novel transcriptional repressor domain, SNAG, and inhibits G1 arrest induced by interleukin-2 withdrawal. Mol Cell Biol 1996; 16: 6263.

10. Tong B, Grimes HL, Yang TY, et al. The Gfi-1B proto-oncoprotein represses p21WAF1 and inhibits myeloid cell differentiation. Mol Cell Biol 1998; 18: 2462.

11. Yucel R, Karsunky H, Klein-Hitpass L, Moroy T. The transcriptional repressor Gfil affects development of early, uncommitted c-Kit+ T cell progenitors and CD4/CD8 lineage decision in the thymus. J Exp Med 2003; 197: 831.

12. Person RE, Li FQ, Duan Z, et al. Gfi1 Proto-Oncogene Mutation Causes Human Neutropenia and Targets Neutrophil Elastase. Nat.Genet. 2003; 
13. Duan Z, Horwitz M. Targets of the transcriptional repressor oncoprotein Gfi-1. Proc Natl.Acad.Sci.U.S.A 2003; 100: 5932.

14. Jegalian $\mathrm{AG}, \mathrm{Wu} \mathrm{H}$. Regulation of Socs gene expression by the proto-oncoprotein GFI-1B: two routes for STAT5 target gene induction by erythropoietin. J Biol.Chem. 2002; 277: 2345.

15. Zweidler-McKay PA, Grimes HL, Flubacher MM, Tsichlis PN. Gfi-1 encodes a nuclear zinc finger protein that binds DNA and functions as a transcriptional repressor. Mol Cell Biol 1996; 16: 4024.

16. Hock H, Hamblen MJ, Rooke HM, et al. Intrinsic requirement for zinc finger transcription factor gfi-1 in neutrophil differentiation. Immunity. 2003; 18: 109.

17. Karsunky $\mathrm{H}$, Zeng $\mathrm{H}$, Schmidt $\mathrm{T}$, et al. Inflammatory reactions and severe neutropenia in mice lacking the transcriptional repressor Gfi1. Nat.Genet. 2002; $\therefore$

18. Saleque $\mathrm{S}$, Cameron $\mathrm{S}$, Orkin $\mathrm{SH}$. The zinc-finger proto-oncogene Gfi-1b is essential for development of the erythroid and megakaryocytic lineages. Genes Dev. 2002; 16: 301. 
19. Doan LL, Kitay MK, Yu Q, et al. Growth factor independence-1B expression leads to defects in T cell activation, IL-7 receptor alpha expression, and T cell lineage commitment. J Immunol 2003; 170: 2356.

20. Rodel B, Tavassoli K, Karsunky H, et al. The zinc finger protein Gfi-1 can enhance STAT3 signaling by interacting with the STAT3 inhibitor PIAS3. EMBO J 2000; 19: 5845.

21. Karsunky H, Mende I, Schmidt T, Moroy T. High levels of the onco-protein Gfi-1 accelerate T-cell proliferation and inhibit activation induced T-cell death in Jurkat T-cells. Oncogene 2002; 21: 1571.

22. Mitchell T, Kappler J, Marrack P. Bystander virus infection prolongs activated T cell survival. J Immunol 1999; 162: 4527.

23. Shao H, Kono DH, Chen LY, Rubin EM, Kaye J. Induction of the early growth response (Egr) family of transcription factors during thymic selection. J Exp Med 1997; 185: 731 .

24. Vindelov LL, Christensen IJ, Nissen NI. A detergent-trypsin method for the preparation of nuclei for flow cytometric DNA analysis. Cytometry 1983; $3: 323$.

25. Hopwood D. Cell and tissue fixation, 1972-1982. Histochem.J 1985; 17: 389. 
26. Veis DJ, Sentman CL, Bach EA, Korsmeyer SJ. Expression of the Bcl-2 protein in murine and human thymocytes and in peripheral T lymphocytes. J Immunol $1993 ; 151: 2546$.

27. Cao XM, Koski RA, Gashler A, et al. Identification and characterization of the Egr-1 gene product, a DNA-binding zinc finger protein induced by differentiation and growth signals. Mol.Cell Biol. 1990; 10: 1931.

28. Lo D, Sprent J. Identity of cells that imprint H-2-restricted T-cell specificity in the thymus. Nature 1986; 319: 672.

29. Anderson G, Jenkinson EJ, Moore NC, Owen JJ. MHC class II-positive epithelium and mesenchyme cells are both required for T-cell development in the thymus. Nature 1993; 362: 70.

30. Schwartz RH. Acquisition of immunologic self-tolerance. Cell 1989; 57: 1073.

31. Zornig M, Schmidt T, Karsunky H, Grzeschiczek A, Moroy T. Zinc finger protein GFI-1 cooperates with myc and pim-1 in T-cell lymphomagenesis by reducing the requirements for IL-2. Oncogene 1996; 12: 1789.

32. Perez P, Lira SA, Bravo R. Overexpression of RelA in transgenic mouse thymocytes: specific increase in levels of the inhibitor protein I kappa B alpha. Mol Cell Biol 1995; 15: 3523. 
33. Strasser A, Harris AW, Cory S. bcl-2 transgene inhibits T cell death and perturbs thymic self-censorship. Cell 1991; 67: 889.

34. Herblot S, Aplan PD, Hoang T. Gradient of E2A activity in B-cell development. Mol.Cell Biol. 2002; 22: 886.

35. Herblot S, Steff AM, Hugo P, Aplan PD, Hoang T. SCL and LMO1 alter thymocyte differentiation: inhibition of E2A-HEB function and pre-T alpha chain expression. Nat.Immunol. 2000; 1: 138.

36. Schmidt T, Karsunky H, Rodel B, Zevnik B, Elsasser HP, Moroy T. Evidence implicating Gfi-1 and Pim-1 in pre-T-cell differentiation steps associated with beta-selection. EMBO J 1998; 17: 5349.

37. Brugnera $\mathrm{E}$, Bhandoola $\mathrm{A}$, Cibotti $\mathrm{R}$, et al. Coreceptor reversal in the thymus: signaled CD4+8+ thymocytes initially terminate CD8 transcription even when differentiating into CD8+ T cells [In Process Citation]. Immunity 2000; 13: 59.

38. Shinkai Y, Rathbun G, Lam KP, et al. RAG-2-deficient mice lack mature lymphocytes owing to inability to initiate V(D)J rearrangement. Cell 1992; 68: 855. 
39. Lucas $\mathrm{B}$, Germain $\mathrm{RN}$. Unexpectedly complex regulation of $\mathrm{CD} 4 / \mathrm{CD} 8$ coreceptor expression supports a revised model for CD4+CD8+ thymocyte differentiation. Immunity. 1996; 5: 461.

40. Robey E, Fowlkes BJ. Selective events in T cell development. Annu.Rev.Immunol 1994; 12:675-705.: 675.

41. Punt JA, Suzuki H, Granger LG, Sharrow SO, Singer A. Lineage commitment in the thymus: only the most differentiated (TCRhibcl-2hi) subset of CD4+CD8+ thymocytes has selectively terminated CD4 or CD8 synthesis. J.Exp.Med. 1996; 184: 2091.

42. Kisielow P, Bluthmann H, Staerz UD, Steinmetz M, von Boehmer H. Tolerance in T-cell-receptor transgenic mice involves deletion of nonmature CD4+8+ thymocytes. Nature 1988; 333: 742.

43. Takahama Y, Shores EW, Singer A. Negative selection of precursor thymocytes before their differentiation into CD4+CD8+ cells. Science 1992; 258: 653.

44. Yoder J, Pham C, Iizuka YM, et al. Requirement for the SLP-76 adaptor GADS in T cell development. Science 2001; 291: 1987.

45. Saijo K, Park SY, Ishida Y, Arase H, Saito T. Crucial role of Jak3 in negative selection of self-reactive T cells. J Exp Med 1997; 185: 351 . 
46. Rincon $\mathrm{M}$, Whitmarsh $\mathrm{A}$, Yang DD, et al. The JNK pathway regulates the In vivo deletion of immature CD4(+)CD8(+) thymocytes. J Exp Med 1998; 188: 1817.

47. Schluns KS, Kieper WC, Jameson SC, Lefrancois L. Interleukin-7 mediates the homeostasis of naive and memory CD8 T cells in vivo. Nat.Immunol 2000; 1: 426.

48. Webb LM, Foxwell BM, Feldmann M. Putative role for interleukin-7 in the maintenance of the recirculating naive CD4+ T-cell pool. Immunology 1999; 98 : 400.

49. Vivien L, Benoist C, Mathis D. T lymphocytes need IL-7 but not IL-4 or IL-6 to survive in vivo. Int.Immunol 2001; $13: 763$.

50. Tan JT, Dudl E, LeRoy E, et al. IL-7 is critical for homeostatic proliferation and survival of naive T cells. Proc Natl.Acad.Sci.U.S.A 2001; 98: 8732.

51. Freeden-Jeffry U, Solvason N, Howard M, Murray R. The earliest T lineagecommitted cells depend on IL-7 for Bcl-2 expression and normal cell cycle progression. Immunity 1997; 7: 147.

52. Strasser A, Harris AW, Corcoran LM, Cory S. Bcl-2 expression promotes B- but not T-lymphoid development in scid mice. Nature 1994; 368: 457. 
53. Linette GP, Grusby MJ, Hedrick SM, Hansen TH, Glimcher LH, Korsmeyer SJ. Bcl-2 is upregulated at the $\mathrm{CD} 4+\mathrm{CD} 8+$ stage during positive selection and promotes thymocyte differentiation at several control points. Immunity. 1994; 1: 197.

54. Akashi K, Kondo M, Freeden-Jeffry U, Murray R, Weissman IL. Bcl-2 rescues T lymphopoiesis in interleukin-7 receptor-deficient mice. Cell 1997; 89: 1033.

55. D'Ambrosio D, Cantrell DA, Frati L, Santoni A, Testi R. Involvement of p21 ras activation in T cell CD69 expression. Eur J Immunol 1994; 24: 616.

56. Porter BO, Scibelli P, Malek TR. Control of T cell development in vivo by subdomains within the IL-7 receptor alpha-chain cytoplasmic tail. J Immunol $2001 ; 166: 262$.

57. Maraskovsky E, O'Reilly LA, Teepe M, Corcoran LM, Peschon JJ, Strasser A. Bcl-2 can rescue T lymphocyte development in interleukin-7 receptor-deficient mice but not in mutant rag-1-/- mice. Cell 1997; 89: 1011.

58. Yasutomo K, Doyle C, Miele L, Germain RN. The duration of antigen receptor signalling determines CD4+ versus CD8+ T-cell lineage fate. Nature 2000; 404: 506. 
59. Wilkinson B, Kaye J. Requirement for sustained MAPK signaling in both CD4 and CD8 lineage commitment: a threshold model. Cell Immunol 2001; 211: 86.

60. Matechak EO, Killeen N, Hedrick SM, Fowlkes BJ. MHC class II-specific T cells can develop in the CD8 lineage when CD4 is absent. Immunity 1996; 4: 337.

61. Penninger JM, Sirard C, Mittrucker HW, et al. The interferon regulatory transcription factor IRF-1 controls positive and negative selection of CD8+ thymocytes. Immunity. 1997; 7: 243.

62. Grimes HL, Gilks CB, Chan TO, Porter S, Tsichlis PN. The Gfi-1 protooncoprotein represses Bax expression and inhibits T-cell death. Proc Natl Acad Sci U.S A. 1996; 93: 14569.

63. Hawley TS, Telford WG, Ramezani A, Hawley RG. Four-color flow cytometric detection of retrovirally expressed red, yellow, green, and cyan fluorescent proteins. Biotechniques 2001; 30: 1028.

64. Kinoshita S, Chen BK, Kaneshima H, Nolan GP. Host control of HIV-1 parasitism in T cells by the nuclear factor of activated T cells. Cell 1998; 95: 595.

65. Jordan M, Schallhorn A, Wurm FM. Transfecting mammalian cells: optimization of critical parameters affecting calcium-phosphate precipitate formation. Nucleic Acids Res. 1996; 24: 596. 
66. Porter S, Larue L, Mintz B. Mosaicism of tyrosinase-locus transcription and chromatin structure in dark vs. light melanocyte clones of homozygous chinchillamottled mice. Dev.Genet. 1991; 12: 393.

67. Dignam JD, Lebovitz RM, Roeder RG. Accurate transcription initiation by RNA polymerase II in a soluble extract from isolated mammalian nuclei. Nucleic.Acids.Res 1983; 11: 1475.

68. Scheijen B, Jonkers J, Acton D, Berns A. Characterization of pal-1, a common proviral insertion site in murine leukemia virus-induced lymphomas of c-myc and Pim-1 transgenic mice. J Virol. 1997; 71: 9.

69. Javahery R, Khachi A, Lo K, Zenzie-Gregory B, Smale ST. DNA sequence requirements for transcriptional initiator activity in mammalian cells. Mol.Cell Biol. 1994; 14: 116.

70. Smale ST, Jain A, Kaufmann J, Emami KH, Lo K, Garraway IP. The initiator element: a paradigm for core promoter heterogeneity within metazoan proteincoding genes. Cold Spring Harb.Symp.Quant.Biol. 1998; 63: 21.

71. Roberts T, Cowell JK. Cloning of the human Gfi-1 gene and its mapping to chromosome region 1p22. Oncogene 1997; 14: 1003. 
72. Liu S, Cowell JK. Cloning and characterization of the TATA-less promoter from the human GFI1 proto-oncogene. Ann.Hum.Genet. 2000; 64: 83.

73. Quandt K, Frech K, Karas H, Wingender E, Werner T. MatInd and MatInspector: new fast and versatile tools for detection of consensus matches in nucleotide sequence data. Nucleic Acids Res. 1995; 23: 4878.

74. Sundstrom C, Nilsson K. Establishment and characterization of a human histiocytic lymphoma cell line (U-937). Int.J Cancer 1976; 17: 565.

75. Nolo R, Abbott LA, Bellen HJ. Senseless, a $\mathrm{Zn}$ finger transcription factor, is necessary and sufficient for sensory organ development in Drosophila [In Process Citation]. Cell 2000; 102: 349.

76. Schmidt T, Karsunky H, Gau E, Zevnik B, Elsasser HP, Moroy T. Zinc finger protein GFI-1 has low oncogenic potential but cooperates strongly with pim and myc genes in T-cell lymphomagenesis. Oncogene 1998; 17: 2661.

77. Jia Y, Xie G, McDermott JB, Aamodt E. The C. elegans gene pag-3 is homologous to the zinc finger proto- oncogene gfi-1. Development 1997; 124 : 2063.

78. Borregaard N, Cowland JB. Granules of the human neutrophilic polymorphonuclear leukocyte. Blood 1997; 89: 3503. 
79. Horwitz M, Benson KF, Duan Z, et al. Role of neutrophil elastase in bone marrow failure syndromes: molecular genetic revival of the chalone hypothesis. Curr.Opin.Hematol 2003; 10: 49.

80. Kyewski B, Derbinski J, Gotter J, Klein L. Promiscuous gene expression and central T-cell tolerance: more than meets the eye. Trends Immunol 2002; 23: 364.

81. Hogquist KA. Signal strength in thymic selection and lineage commitment. Curr.Opin.Immunol 2001; 13: 225.

82. Singer A, Bosselut R, Bhandoola A. Signals involved in CD4/CD8 lineage commitment: current concepts and potential mechanisms. Semin Immunol 1999; 11: 273 .

83. Kaye J, Vasquez NJ, Hedrick SM. Involvement of the same region of the T cell antigen receptor in thymic selection and foreign peptide recognition. J Immunol. 1992; 148: 3342.

84. Kaye J, Hsu ML, Sauron ME, Jameson SC, Gascoigne NR, Hedrick SM. Selective development of CD4+ T cells in transgenic mice expressing a class II MHC-restricted antigen receptor. Nature 1989; 341: 746. 
85. Murphy KM, Heimberger AB, Loh DY. Induction by antigen of intrathymic apoptosis of CD4+CD8+TCRlo thymocytes in vivo. Science 1990; 250: 1720.

86. Shinkai Y, Koyasu S, Nakayama K, et al. Restoration of T cell development in RAG-2-deficient mice by functional TCR transgenes. Science 1993; 259: 822.

87. Gabor MJ, Godfrey DI, Scollay R. Recent thymic emigrants are distinct from most medullary thymocytes. Eur J Immunol 1997; 27: 2010.

88. Bradley LM, Watson SR, Swain SL. Entry of naive CD4 T cells into peripheral lymph nodes requires L-selectin. J Exp Med 1994; 180: 2401.

89. Nakayama T, Kasprowicz DJ, Yamashita M, et al. The generation of mature, single-positive thymocytes in vivo is dysregulated by CD69 blockade or overexpression. J Immunol 2002; 168: 87.

90. Feng C, Woodside KJ, Vance BA, et al. A potential role for CD69 in thymocyte emigration. Int.Immunol 2002; 14: 535.

91. Corn PG, El Deiry WS. Derangement of growth and differentiation control in oncogenesis. Bioessays 2002; 24: 83.

92. Cillo C, Cantile M, Faiella A, Boncinelli E. Homeobox genes in normal and malignant cells. J Cell Physiol 2001; 188: 161. 
93. Murre C. Intertwining proteins in thymocyte development and cancer. Nat.Immunol 2000; 1: 97.

94. Peifer M, Polakis P. Wnt signaling in oncogenesis and embryogenesis--a look outside the nucleus. Science 2000; 287: 1606.

95. Alvarez M, Rhodes SJ, Bidwell JP. Context-dependent transcription: all politics is local. Gene 2003; 313: 43.

96. Merika M, Thanos D. Enhanceosomes. Curr.Opin.Genet.Dev. 2001; 11: 205.

97. Carey M. The enhanceosome and transcriptional synergy. Cell 1998; 92: 5 .

98. Greenhalgh CJ, Hilton DJ. Negative regulation of cytokine signaling. J Leukoc.Biol. 2001; 70: 348.

99. Nakagawa K, Yokosawa H. PIAS3 induces SUMO-1 modification and transcriptional repression of IRF-1. FEBS Lett. 2002; 530: 204.

100. Kotaja N, Karvonen U, Janne OA, Palvimo JJ. PIAS proteins modulate transcription factors by functioning as SUMO-1 ligases. Mol.Cell Biol. 2002; 22: 5222.

101. Seeler JS, Dejean A. Nuclear and unclear functions of SUMO. Nat.Rev.Mol.Cell Biol. 2003; 4: 690. 


\section{LIST OF ABBREVIATIONS}

TCR; T cell receptor

MHC; Major histocompatibility complex

GFI1; Growth factor independence-1

GFI1B; Growth factor independence-1B

IL7; Interleukin 7

IL7R; Interleukin 7 receptor

$\mathrm{NK}$; Natural killer

RAG; Recombinase activating gene

DN; Double negative

DP; Double positive

SP; Single positive

EGR1; Early growth response-1

NFAT; Nuclear factor of activated T cells

NFкB; Nuclear factor kappa B

AP-1; Activator protein 1

BCL2; B cell lymphoma 2

MAPK; Mitogen activated protein kinase

IL-2; Interleukin 2

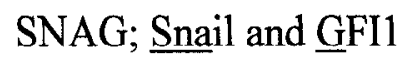


TRAF5; TNFR-associated factor 5

LKLF; Lung Kruppel like factor

ID; Inhibitor of DNA binding

ChIP; Chromatin immunoprecipitation

SOCS; Suppressor of cytokine signaling

FACS; Fluorescence activated cell sorting

RT-PCR; Reverse transcriptase-polymerase chain reaction

WT; Wild type

IRF-1; Interferon regulatory factor-1

SEM; Standard error of the mean 
Curriculum Vitae

Loretta L. Doan

N.S.F. Graduate Research Fellow

Ph.D. Candidate, Biochemistry and Molecular Biology

Member, Institute for Cellular Therapeutics

University of Louisville

570 South Preston Street, Room 420

Louisville, KY 40202

\section{EDUCATION}

1999-2004

Graduate program, Dept. of Biochemistry and Molecular Biology

University of Louisville, Louisville, KY

Advisor: H. Leighton Grimes, Ph.D.

1999

B.A. Chemistry, Indiana University Southeast

1993

B.A. Communications/Theatre, Indiana University Southeast

\section{FELLOWSHIPS}

2001-2004

National Science Foundation Graduate Research Fellowship

1999-2001

University Fellowship, University of Louisville 


\section{AWARDS AND HONORS}

1996-1999

1997-1999

1998-1999

1998-1999
Chancellor's/Dean's list

IUS Merit Scholarship

Local American Chemical Society Award for Outstanding

Academic Performance

IUS Outstanding Chemistry Student of the Year

\section{PROFESSIONAL MEMBERSHIPS}

2001-2002

Associate member of the American Association of Cancer

Researchers

1999-2000

Student member of the American Chemical Society

\section{TEACHING RESPONSIBILITIES}

Spring, $2001 \quad$ Biochemistry II, T.A.

1997-1998 Teaching assistant, chemistry lab

\section{COMMUNITY SERVICE}

2001-2003 Volunteer for Recording for the Blind and Dyslexic

2002 Volunteer for Cancer Survivor Day at Brown Cancer Center 


\section{SERVICE TO THE UNIVERSITY OF LOUISVILLE}

2001

Student representative to the Graduate Executive Committee

\section{TRAINING AND RESEARCH EXPERIENCE}

2000-2004 GFI1 and GFI1B in T cell activation and development

July, 2001

AACR Pathobiology of Cancer Workshop

Summer, $1998 \quad$ University of Nevada, Reno, Summer Undergraduate Research

Program; organic synthesis

\section{PUBLICATIONS-Peer Reviewed}

1. Doan, L.L., Kitay. M.K., Yu, Q., Singer, A., Herblot, S., Hoang, T., Bear, S.E., Morse, H.C., Tsichlis, P.N., and Grimes, H.L. Growth Factor Independence 1B expression leads to defects in T-cell activation, IL-7R $\alpha$ expression and T-cell lineage commitment. (The Journal of Immunology, 2003 March 1, 170(5), 235666.)

2. Doan, L.L., Tanner, M.K., and Grimes, H.L. Intranuclear staining of proteins in heterogeneous cell populations and verification of nuclear localization by flow cytometric analysis. (Journal of Immunological Methods, 279 (2003) 193-198.)

3. Doan, L.L., Gilks, C.B., Visser J.W.M., Porter, S., Flubacher, S., and Grimes, H.L. Targeted repression of Gfi1 by GFI1 and GFI1B in T cells. (Manuscript submitted.) 


\section{NATIONAL PRESENTATIONS}

1. Doan LL, Kitay MK, Grimes HL. GFI1B replaces GFI1, altering CD4 SP egress and T-cell homeostasis. (Poster) Keystone Symposium on T Lymphocyte Activation, Differentiation and Death, Keystone, Colorado, Jan. 16-22, 2002.

2. Doan LL, Gilks CB, Porter SD, Visser JWM, Bear SE, Morse HC, Tsichlis PN, Grimes HL. GFI1 is a direct target of GFIl and GFI1B repression. (Oral Presentation) ThymUS, San Juan, Puerto Rico, Nov. 2-7, 2001.

3. Doan LL, Kitay MK, Grimes HL. GFI1B replaces GFI1 and alters CD4 SP egress and T-cell homeostasis. (Oral Presentation) ThymUS, San Juan, Puerto Rico, Nov. 2-7, 2001.

4. Doan LL, Gilks CB, Porter SD, Visser JWM, Bear SE, Morse HC, Tsichlis PN, Grimes HL. GFI1 is autoregulatory in T cells. (Poster) Pathobiology of cancer workshop, Keystone, Colorado, July 15-22, 2001. 